\title{
Copper-Catalyzed Chloro-Arylsulfonylation of Styrene Derivatives via the Insertion of Sulfur Dioxide
}

Yue Li, ${ }^{\dagger}$ Lin Shen, ${ }^{\dagger}$ Mi Zhou, ${ }^{\dagger}$ Baojian Xiong, ${ }^{\dagger}$ Xuemei Zhang ${ }^{\dagger *}$ and Zhong Lian ${ }^{\dagger *}$ ${ }^{\dagger}$ Department of Dermatology, State Key Laboratory of Biotherapy and Cancer Center, National Clinical Research Center for Geriatrics, West China Hospital and West China School of Pharmacy, Sichuan University, Chengdu 610041 (P.R. China)

\section{Contents}

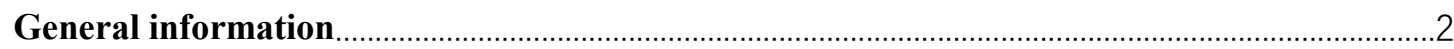

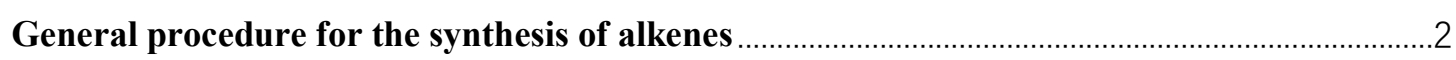

General procedure for the synthesis of vicinal chloro-arylsulfonylated compounds ...................

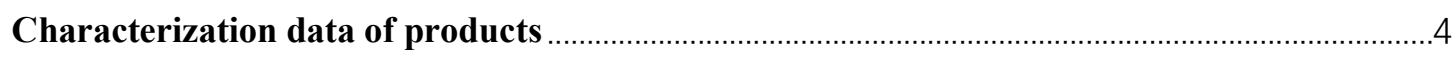

Control experiments using other halide salts.

Gram-scale reaction and transformations of $\boldsymbol{\beta}$-chloroalkyl sulfones. ........................................ 12

Reference

Copies of ${ }^{1} \mathrm{H}$ NMR, ${ }^{19} \mathrm{~F}$ NMR and ${ }^{13} \mathrm{C}$ NMR spectra of Products ......................................... 15 


\section{General information}

Unless otherwise noted, all reactions or reagents were obtained from commercial suppliers and used as received. Unless otherwise noted, all catalytic reactions were set up in an argon atmosphere glovebox (Vigor, SGI800-750TS-F). The substrates and reagents for catalytic reactions were degassed and stored in the glovebox, unless otherwise noted. All work-up and purification procedures were carried out with reagent-grade solvents in air.

Thin Layer Chromatography analysis was performed on silica gel coated glass plates $(0.25 \mathrm{~mm})$ with fluorescence indicator UV254. For detection of spots, irradiation of UV light at $254 \mathrm{~nm}$ or staining reagent using phosphomolybdic acid solution was used. Flash column chromatography was conducted with silica gel 60 (particle size 230-400 mesh, Huanghai) at room temperature and under elevated pressure.

Gas chromatography (GC) analysis was conducted on a Shimadzu GC-2030 instrument equipped with a Rtx-5 column $(30 \mathrm{~m} \times 0.25 \mathrm{~mm})$ with dodecane as an internal standard. GC-MS analysis was conducted on a Agilent 5977B GC/MSD instrument equipped with a HP-5MS UI column (30 $\mathrm{m} \times$ $0.25 \mathrm{~mm}$ ). ${ }^{1} \mathrm{H}$ NMR and ${ }^{13} \mathrm{C}$ NMR spectra were recorded at $400 \mathrm{MHz}$ and $101 \mathrm{MHz}$, respectively in $\mathrm{CDCl}_{3}$ (or $\left(\mathrm{CD}_{3}\right)_{2} \mathrm{SO}$ ) at room temperature. ${ }^{1} \mathrm{H}$ NMR was reported as follows: chemical shift, multiplicity $(\mathrm{s}=$ singlet, $\mathrm{d}=$ doublet, $\mathrm{t}=$ triplet, $\mathrm{q}=$ quadruplet, $\mathrm{m}=$ multiplet $)$, coupling constant $(\mathrm{J}$ values) in $\mathrm{Hz}$ and integration. Chemical shifts $(\delta)$ were reported with respect to the corresponding solvent residual peak at $7.26 \mathrm{ppm}$ for $\mathrm{CDCl}_{3}$ (or $2.50 \mathrm{ppm}$ for $\left(\mathrm{CD}_{3}\right)_{2} \mathrm{SO}$ ) for ${ }^{1} \mathrm{H} \mathrm{NMR} .{ }^{13} \mathrm{C} \mathrm{NMR}$ spectra $\left({ }^{1} \mathrm{H}\right.$-broadband decoupled) were reported in ppm using the central peak of $\mathrm{CDCl}_{3}(77.16$ ppm). High-resolution mass spectrometric measurements were provided by the Department of The State Key Laboratory of Biotherapy, Sichuan University. The molecular ion $[\mathrm{M}+\mathrm{H}]^{+},[\mathrm{M}]^{+}$and $[\mathrm{M}+\mathrm{Na}]^{+}$are given in $\mathrm{m} / \mathrm{z}$ units.

\section{General procedure for the synthesis of alkenes}

General procedure $\mathrm{A}^{[1]}$

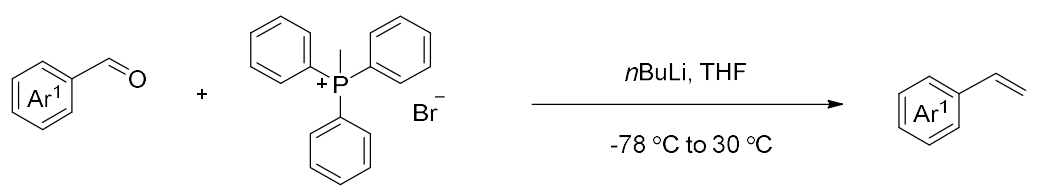

Methyltriphenylphosphonium bromide ( $4.89 \mathrm{~g}, 13.7 \mathrm{mmol}, 1.30$ equiv) was suspended in THF $(20 \mathrm{~mL})$ and $\mathrm{n}-\mathrm{BuLi}(5.45 \mathrm{~mL}$ of a $2.5 \mathrm{M}$ soln., $13.7 \mathrm{mmol}, 1.30$ equiv) was added dropwise with stirring under $\mathrm{N}_{2}$ at $-78^{\circ} \mathrm{C}$. After the reaction was stirred for $1 \mathrm{~h}$ at $30^{\circ} \mathrm{C}$, ArCHO $(10.5 \mathrm{mmol}, 1.00$ equiv) in THF $(5.0 \mathrm{~mL})$ was added dropwise at $-78{ }^{\circ} \mathrm{C}$. Then, the reaction was stirred for $16 \mathrm{~h}$ at 
$30{ }^{\circ} \mathrm{C}$. The reaction was quenched with $\mathrm{H}_{2} \mathrm{O}(20 \mathrm{~mL})$ and extracted 3 times with ether $(20 \mathrm{~mL})$. The combined organic layer was dried over $\mathrm{MgSO}_{4}$. The filtrate was concentrated in vacuo and the residue was purified by chromatography on silica gel.

General procedure $B^{[1]}$

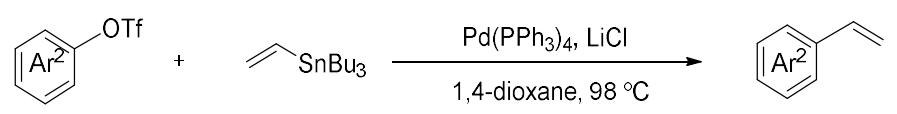

To a solution of ArOTf (18.0 mmol, 1.0 equiv) in $50 \mathrm{~mL}$ of 1,4-dioxane were added tri-nbutylethenylstannane (5.27 mL, $18.0 \mathrm{mmol}, 1.0$ equiv), $\mathrm{LiCl}(2.14 \mathrm{~g}, 50.4 \mathrm{mmol}, 2.8$ equiv), $\mathrm{Pd}\left(\mathrm{PPh}_{3}\right)_{4}$ (416 mg, $0.360 \mathrm{mmol}, 0.02$ equiv), and a few crystals of 2,6-di-tert-butyl-4-methylphenol. The resulting suspension was heated to $98{ }^{\circ} \mathrm{C}$ for $4 \mathrm{~h}$, cooled to room temperature, and treated with $8.7 \mathrm{~mL}$ of pyridine and $17.4 \mathrm{~mL}$ of pyridinium fluoride (1.40 M solution in THF, 24.4 mmol). The resulting mixture was stirred at $23{ }^{\circ} \mathrm{C}$ for $16 \mathrm{~h}$. The mixture was diluted with diethyl ether, filtered through a small pad of Celite, and washed with water, $10 \% \mathrm{HCl}$, water, and a concentrated sodium chloride solution. The combined organic layer was dried over $\mathrm{MgSO}_{4}$. The filtrate was concentrated in vacuo and the residue was purified by chromatography on silica gel.

\section{General procedure for the synthesis of vicinal chloro-arylsulfonylated compounds}

Chamber $\mathrm{A}$ : for $\mathrm{SO}_{2}$ gas generation
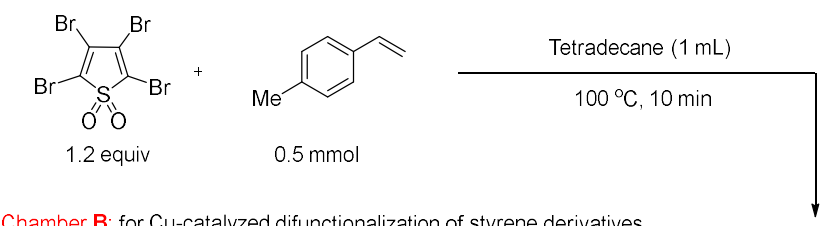

Chamber B: for Cu-catalyzed difunctionalization of styrene derivatives

$\mathrm{SO}_{2}(2.5$ equiv)
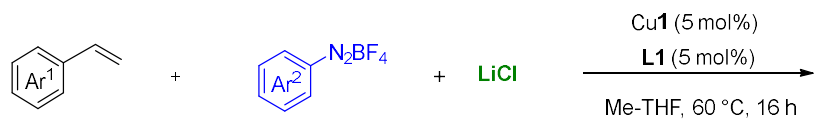

$1,0.2 \mathrm{mmol}$

$2,1.5$ equiv

3, 1.0 equiv
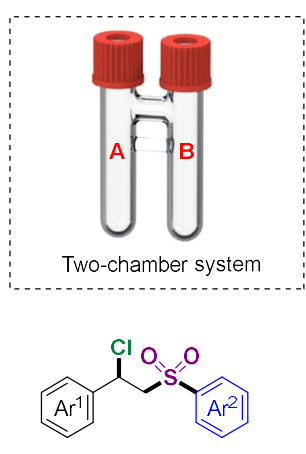

4

In the glovebox, Styrenes ( $0.2 \mathrm{mmol}, 1.0$ equiv), aryldiazonium tetrafluoroborates ( $0.3 \mathrm{mmol}, 1.5$ equiv), $\mathrm{Cu} 1$ (2.1 mg, $5.0 \mathrm{~mol} \%), \mathbf{L 1}$ (2.6 mg, $5.0 \mathrm{~mol} \%), \mathrm{LiCl}(8.5 \mathrm{mg}, 1.0$ equiv) were added to chamber B, followed by addition of Me-THF $(1.0 \mathrm{~mL})$. Tetrabromothiophene S,S-dioxides $(0.6$ mmol, $259.0 \mathrm{mg})$ in tetradecane $(1.0 \mathrm{~mL})$ was added to chamber A, followed by addition of 4methylphenylene $(0.5 \mathrm{mmol}, 62.9 \mu \mathrm{l})$. The two chamber system was sealed and removed out of the glovebox, then chamber A was heated to $100{ }^{\circ} \mathrm{C}$ in heat block. About ten minutes later, the sulfur dioxide was completely released since the system in chamber A became clear. Then chamber B was heated to $60{ }^{\circ} \mathrm{C}$ in heat block. After 16 hours, two chamber was cooled to room temperature. The mixture in chamber B was passed through a short silica gel pad with ethyl acetate. The filtrate was 
washed by ethyl acetate and $\mathrm{H}_{2} \mathrm{O}(15 \mathrm{~mL} \times 3)$, dried by $\mathrm{Na}_{2} \mathrm{SO}_{4}$, then concentrated and the residue was purified by flash column chromatography to give the desired product.

\section{Characterization data of products}<smiles>Cc1ccc(S(=O)(=O)CC(Cl)c2ccccc2)cc1</smiles>

(S)-1-((2-chloro-2-phenylethyl)sulfonyl)-4-methylbenzene (4a) : Prepared by the general procedure at the $60^{\circ} \mathrm{C}$; isolated as white solid using petroleum/ethyl acetate (10:1) as eluent (51.9 mg, 88\%). Known compound ${ }^{[2]} .{ }^{1} \mathrm{H}$ NMR (400 MHz, Chloroform- $d$ ) $\delta 7.56(\mathrm{~d}, J=8.3 \mathrm{~Hz}$, 2H), 7.20 (s, $5 \mathrm{H}), 7.16(\mathrm{~d}, J=8.0 \mathrm{~Hz}, 2 \mathrm{H}), 5.25$ (t, $J=6.9 \mathrm{~Hz}, 1 \mathrm{H}), 3.87$ (dd, $J=14.8,6.9 \mathrm{~Hz}, 1 \mathrm{H}), 3.76$ (dd, $J=14.8,6.9 \mathrm{~Hz}, 1 \mathrm{H}), 2.34$ (s, 3H). ${ }^{13} \mathrm{C}$ NMR $(101 \mathrm{MHz}$, Chloroform- $d$ ) $\delta$ 144.9, 138.6, 136.2, 129.8, 129.1，128.9，128.2，127.2，64.2，55.1，21.6. HRMS (ESI/Q-TOF) $\mathrm{m} / \mathrm{z}:[\mathrm{M}+\mathrm{Na}]^{+}$calcd for $\mathrm{C}_{15} \mathrm{H}_{15} \mathrm{ClNaO}_{2} \mathrm{~S} 317.0373$; found: 317.0373 .

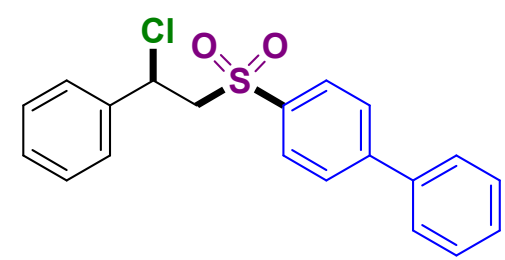

(R)-4-((2-chloro-2-phenylethyl)sulfonyl)-1,1'-biphenyl (4b) : Prepared by the general procedure at the $60{ }^{\circ} \mathrm{C}$; isolated as white solid using petroleum/ethyl acetate (10:1) as eluent (63.5 mg, 89\%). Known compound ${ }^{[3]}{ }^{1} \mathrm{H}$ NMR (400 MHz, Chloroform- $d$ ) $\delta 7.69(\mathrm{~d}$, $J=8.4 \mathrm{~Hz}, 2 \mathrm{H}), 7.53(\mathrm{~d}, J=8.4 \mathrm{~Hz}, 2 \mathrm{H}), 7.50-7.47$ (m, 2H), 7.43 $-7.33(\mathrm{~m}, 3 \mathrm{H}), 7.21-7.15(\mathrm{~m}, 5 \mathrm{H}), 5.30$ (t, $J=7.0 \mathrm{~Hz}, 1 \mathrm{H}), 3.93$ $(\mathrm{dd}, J=14.8,6.6 \mathrm{~Hz}, 1 \mathrm{H}), 3.85(\mathrm{dd}, J=14.8,7.3 \mathrm{~Hz}, 1 \mathrm{H}) .{ }^{13} \mathrm{C}$ NMR $(101 \mathrm{MHz}$, Chloroform- $d$ ) $\delta 146.7$, 139.1, 138.4, 137.6, 129.2, 129.1, 128.9, 128.8, 128.7, 127.7, 127.4, 127.3, 64.1, 55.2. HRMS (ESI/QTOF) $\mathrm{m} / \mathrm{z}:[\mathrm{M}+\mathrm{Na}]^{+}$calcd for $\mathrm{C}_{20} \mathrm{H}_{17} \mathrm{ClNaO}_{2} \mathrm{~S} 379.0530$; found: 379.0534 .<smiles>O=S(=O)(CC(Cl)c1ccccc1)c1cccc2ccccc12</smiles>

(S)-1-((2-chloro-2-phenylethyl)sulfonyl)naphthalene (4c) : Prepared by the general procedure at the $60{ }^{\circ} \mathrm{C}$; isolated as white solid using petroleum/ethyl acetate (10:1) as eluent (42 mg, 64\%). ${ }^{1} \mathrm{H}$ NMR (400 MHz, Chloroform- $d$ ) $\delta 8.63$ (d, $J=8.6 \mathrm{~Hz}, 1 \mathrm{H}), 8.01$ (d, $J=7.8 \mathrm{~Hz}, 2 \mathrm{H}), 7.93$ (d, $J=8.2 \mathrm{~Hz}, 1 \mathrm{H}), 7.73$ (t, $J=7.6 \mathrm{~Hz}, 1 \mathrm{H}), 7.63$ (t, $J=7.5 \mathrm{~Hz}, 1 \mathrm{H}), 7.39$ (t, $J$ $=7.8 \mathrm{~Hz}, 1 \mathrm{H}), 7.16-7.06(\mathrm{~m}, 5 \mathrm{H}), 5.38(\mathrm{t}, J=7.0 \mathrm{~Hz}, 1 \mathrm{H}), 4.15-4.09(\mathrm{~m}$, 2H). ${ }^{13} \mathrm{C}$ NMR (101 MHz, Chloroform- $d$ ) $\delta$ 142.7, 138.1, 135.3, 134.0, 133.8, 131.1, 129.4, 129.1, 129.0, 128.6, 127.03, 126.99, 124.4, 123.6, 63.1, 55.2. HRMS (ESI/Q-TOF) $\mathrm{m} / \mathrm{z}:[\mathrm{M}+\mathrm{Na}]^{+}$calcd for $\mathrm{C}_{18} \mathrm{H}_{15} \mathrm{ClNaO}_{2} \mathrm{~S}$ 353.0373; found: 353.0372 .<smiles>O=S(=O)(CC(Cl)c1ccc(-c2ccccc2)cc1)c1ccc2ccccc2c1</smiles>

(S)-2-((2-([1,1'-biphenyl]-4-yl)-2chloroethyl)sulfonyl)naphthalene (4d): Prepared by the general procedure at the $60{ }^{\circ} \mathrm{C}$; isolated as white solid using petroleum/ethyl acetate (10:1) as eluent (59 mg, 73\%). 1H NMR (400 MHz, Chloroform-d) $\delta 8.16$ (s, 1H), 7.84 - 7.75 (m, 3H), $7.68(\mathrm{dd}, \mathrm{J}=8.7,1.9 \mathrm{~Hz}, 1 \mathrm{H}), 7.64-7.50(\mathrm{~m}, 2 \mathrm{H}), 7.47$ (t, J = 8.2, 6.8, $1.2 \mathrm{~Hz}, 1 \mathrm{H}), 7.38-7.30$ $(\mathrm{m}, 3 \mathrm{H}), 7.28-7.24(\mathrm{~m}, 5 \mathrm{H}), 5.42(\mathrm{t}, \mathrm{J}=7.6 \mathrm{~Hz}, 1 \mathrm{H}), 4.12-3.99(\mathrm{~m}, 2 \mathrm{H}) .{ }^{13} \mathrm{C}$ NMR $(101 \mathrm{MHz}$, 
Chloroform- $d$ ) $\delta 142.1,139.8,136.9,135.8,135.1,131.9,130.4,129.5,129.4,128.7,127.9,127.8,127.7$, 127.4, 127.10, 127.06, 122.4, 63.7, 55.1. HRMS (ESI/Q-TOF) m/z: $[\mathrm{M}+\mathrm{Na}]^{+}$calcd for $\mathrm{C}_{16} \mathrm{H}_{21} \mathrm{NNaO}_{3} \mathrm{~S}$ 330.1134; found: 330.1139 .<smiles>Cc1ccc(S(=O)(=O)CC(Cl)c2ccccc2)cc1C</smiles>

\section{(S)-4-((2-chloro-2-phenylethyl)sulfonyl)-1,2-dimethylbenzene}

(4e) : Prepared by the general procedure at the $60{ }^{\circ} \mathrm{C}$; isolated as white solid using petroleum/ethyl acetate (10:1) as eluent (42 $\mathrm{mg}$, $68 \%) .{ }^{1} \mathrm{H}$ NMR (400 MHz, Chloroform- $d$ ) $\delta 7.51(\mathrm{~d}, J=8.0,2.1 \mathrm{~Hz}$,

1H), $7.45(\mathrm{~d}, J=2.1 \mathrm{~Hz}, 1 \mathrm{H}), 7.29(\mathrm{~s}, 5 \mathrm{H}), 7.22(\mathrm{~d}, J=7.9 \mathrm{~Hz}, 1 \mathrm{H}), 5.35$ (t, $J=7.0 \mathrm{~Hz}, 1 \mathrm{H}), 4.00-3.83$ (m, 2H), 2.32 (s, 3H), 2.27 (s, 3H). ${ }^{13} \mathrm{C}$ NMR (101 MHz, Chloroform-d) $\delta 143.6,138.6,137.9,136.3$, 130.3, 129.03, 128.95, 128.8 127.2, 125.6, 64.2, 55.2, 20.03, 19.74. HRMS (ESI/Q-TOF) m/z: [M+Na] ${ }^{+}$ calcd for $\mathrm{C}_{16} \mathrm{H}_{17} \mathrm{ClNaO}_{2} \mathrm{~S} 331.0530$; found: 331.0528 .

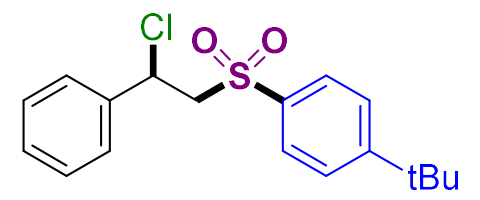

1-(tert-butyl)-4-((2-chloro-2-phenylethyl)sulfonyl)benzene (4f) : Prepared by the general procedure at the $60^{\circ} \mathrm{C}$; isolated as white solid using petroleum/ethyl acetate (10:1) as eluent $(50.5 \mathrm{mg}, 75 \%) .{ }^{1} \mathrm{H}$ NMR (400 MHz, Chloroform- $d) \delta 7.61(\mathrm{~d}, J=8.8 \mathrm{~Hz}, 2 \mathrm{H}), 7.41(\mathrm{~d}, J$ $=8.8 \mathrm{~Hz}, 2 \mathrm{H}), 7.26-7.20(\mathrm{~m}, 5 \mathrm{H}), 5.35(\mathrm{dd}, J=7.6,6.8 \mathrm{~Hz}, 1 \mathrm{H}), 3.95(\mathrm{dd}, J=14.8,6.8 \mathrm{~Hz}, 1 \mathrm{H}), 3.88$ (dd, $J=14.8,7.6 \mathrm{~Hz}, 1 \mathrm{H}), 1.31(\mathrm{~s}, 9 \mathrm{H}) .{ }^{13} \mathrm{C}$ NMR (101 MHz, Chloroform- $d$ ) $\delta 157.7,138.4,136.0$, 129.1, 128.8, 127.9, 127.2, 126.1, 64.0, 55.1, 35.2, 31.0. HRMS (ESI/Q-TOF) m/z: $[\mathrm{M}+\mathrm{K}]^{+}$calcd for $\mathrm{C}_{18} \mathrm{H}_{21} \mathrm{ClKO}_{2} \mathrm{~S} 375.0582$; found: 375.0581 .<smiles>COc1ccc(S(=O)(=O)CC(Cl)c2ccccc2)cc1</smiles>

(R)-1-((2-chloro-2-phenylethyl)sulfonyl)-4-methoxybenzene (4g): Prepared by the general procedure at the $60{ }^{\circ} \mathrm{C}$; isolated as white solid using petroleum/ethyl acetate (5:1) as eluent (57.8 $\mathrm{mg}, 93 \%)$. Known compound ${ }^{[2]} .{ }^{1} \mathrm{H}$ NMR (400 MHz, Chloroform- $d$ ) $\delta 7.59$ (d, $\left.J=8.8 \mathrm{~Hz}, 2 \mathrm{H}\right), 7.19$ (s, 5H), 6.81 $(\mathrm{d}, J=8.8 \mathrm{~Hz}, 2 \mathrm{H}), 5.24(\mathrm{t}, J=6.8 \mathrm{~Hz}, 1 \mathrm{H}), 3.86(\mathrm{dd}, J=14.4,6.8 \mathrm{~Hz}, 1 \mathrm{H}), 3.79-3.72(\mathrm{~m}, 5 \mathrm{H}) .{ }^{13} \mathrm{C}$ NMR (101 MHz, Chloroform- $d$ ) $\delta 163.8,138.7,130.6,130.4$, 129.1, 128.9, 127.2, 114.4, 64.3, 55.7, 55.3. HRMS (ESI/Q-TOF) m/z: [M+Na] $]^{+}$calcd for $\mathrm{C}_{15} \mathrm{H}_{15} \mathrm{ClNaO}_{3} \mathrm{~S} 333.0323$; found: 333.0323 .<smiles>O=S(=O)(CC(Cl)c1ccc2ccccc2c1)c1ccc(OC(F)(F)F)cc1</smiles>

(S)-2-(1-chloro-2-((4(trifluoromethoxy)phenyl)sulfonyl)ethyl)naphthalene (4h) : Prepared by the general procedure at the $60{ }^{\circ} \mathrm{C}$; isolated as white solid using petroleum/ethyl acetate $(10: 1)$ as eluent (49.8 mg, 60\%). ${ }^{1} \mathrm{H}$ NMR (400 MHz, Chloroform- $d$ ) $\delta 7.79-7.72(\mathrm{~m}, 3 \mathrm{H}), 7.67-7.57$ (m, 3H), $7.54-$ $7.48(\mathrm{~m}, 2 \mathrm{H}), 7.25-7.22(\mathrm{~m}, 1 \mathrm{H}), 6.97(\mathrm{~d}, J=7.9 \mathrm{~Hz}, 2 \mathrm{H}), 5.52(\mathrm{dd}, J=7.9,6.4 \mathrm{~Hz}, 1 \mathrm{H}), 4.08(\mathrm{dd}, J=$ 14.9, 6.4 Hz, 1H), 4.02 (dd, $J=14.9,8.0 \mathrm{~Hz}, 1 \mathrm{H}) .{ }^{19} \mathrm{~F}$ NMR $\left(376 \mathrm{MHz}\right.$, Chloroform-d) $\delta-57.71 .{ }^{13} \mathrm{C}$ NMR (101 MHz, Chloroform- $d$ ) $\delta 152.8$ (q, $J=2.0 \mathrm{~Hz}), 137.1,134.7,133.4,132.7,130.3,129.2,128.0$, 127.7, 127.24, 127.20, 127.0, 123.6, 120.4, 120.0 (q, $J=260.0 \mathrm{~Hz}$ ), 63.9, 55.4. HRMS (ESI/Q-TOF) m/z: $[\mathrm{M}+\mathrm{Na}]^{+}$calcd for $\mathrm{C}_{19} \mathrm{H}_{14} \mathrm{ClF}_{3} \mathrm{NaO}_{3} \mathrm{~S}$ 437.0196; found: 437.0197 . 
<smiles>O=S(=O)(CC(Cl)c1ccccc1)c1ccc(Oc2ccccc2)cc1</smiles>

(R)-1-((2-chloro-2-phenylethyl)sulfonyl)-4-phenoxybenzene (4i) : Prepared by the general procedure at the $60^{\circ} \mathrm{C}$; isolated as white solid using petroleum/ethyl acetate $(10: 1)$ as eluent (50.5 mg, 92\%). ${ }^{1} \mathrm{H}$ NMR (400 MHz, Chloroform- $d$ ) $\delta 7.57$ (d, $J=8.6 \mathrm{~Hz}, 2 \mathrm{H}), 7.34(\mathrm{t}, J=7.8 \mathrm{~Hz}, 2 \mathrm{H}), 7.22-7.16(\mathrm{~m}, 5 \mathrm{H}), 6.96(\mathrm{~d}, J=8.0 \mathrm{~Hz}, 2 \mathrm{H}), 6.84(\mathrm{~d}, J=8.7$ $\mathrm{Hz}, 2 \mathrm{H}), 5.26(\mathrm{t}, J=7.0 \mathrm{~Hz}, 1 \mathrm{H}), 3.88(\mathrm{dd}, J=14.8,6.7 \mathrm{~Hz}, 1 \mathrm{H}), 3.79(\mathrm{dd}, J=14.8,7.2 \mathrm{~Hz}, 1 \mathrm{H}) .{ }^{13} \mathrm{C}$ NMR (101 MHz, Chloroform- $d$ ) $\delta 162.6,154.8,138.5,132.5,130.5,130.3,129.2,129.0,127.3,125.2$, 120.4, 117.5, 64.2, 55.2. HRMS (ESI/Q-TOF) m/z: [M+Na] calcd for $\mathrm{C}_{20} \mathrm{H}_{17} \mathrm{ClNaO}_{3} \mathrm{~S} 395.0479$; found: 395.0477.<smiles>O=S(=O)(CC(Cl)c1ccccc1)c1ccc(/N=N/c2ccccc2)cc1</smiles>

(R,E)-1-(4-((2-chloro-2-phenylethyl)sulfonyl)phenyl)-2phenyldiazene (4j) : Prepared by the general procedure at the $60^{\circ} \mathrm{C}$; isolated as orange solid using petroleum/ethyl acetate (2:1) as eluent $(60.0 \mathrm{mg}, 78 \%) .{ }^{1} \mathrm{H}$ NMR $(400 \mathrm{MHz}$,

Chloroform- $d$ ) $\delta 7.91-7.87(\mathrm{~m}, 2 \mathrm{H}), 7.84(\mathrm{~d}, J=8.7 \mathrm{~Hz}, 2 \mathrm{H}), 7.79(\mathrm{~d}, J=8.7 \mathrm{~Hz}, 2 \mathrm{H}), 7.53-7.43(\mathrm{~m}$, 3H), $7.25-7.15$ (m, 5H), 5.31 (t, $J=6.9 \mathrm{~Hz}, 1 \mathrm{H}), 3.96(\mathrm{dd}, J=14.9,6.9 \mathrm{~Hz}, 1 \mathrm{H}), 3.85$ (dd, $J=14.9,7.0$ $\mathrm{Hz}, 1 \mathrm{H}){ }^{13} \mathrm{C}$ NMR (101 MHz, Chloroform- $d$ ) $\delta$ 155.3, 152.4, 140.4, 138.3, 132.3, 129.35, 129.33, 129.31, 129.0, 127.2, 123.4, 123.2, 64.2, 55.1. HRMS (ESI/Q-TOF) m/z: $[\mathrm{M}+\mathrm{Na}]^{+}$calcd for $\mathrm{C}_{20} \mathrm{H}_{18} \mathrm{ClN}_{2} \mathrm{NaO}_{2} \mathrm{~S}$ 385.0772; found: 385.0787 .<smiles>O=S(=O)(CC(Cl)c1ccccc1)c1ccc(F)cc1</smiles>

\section{(R)-1-((2-chloro-2-phenylethyl)sulfonyl)-4-fluorobenzene (4k) :}

Prepared by the general procedure at the $60{ }^{\circ} \mathrm{C}$; isolated as yellow solid using petroleum/ethyl acetate (10:1) as eluent $(43.0 \mathrm{mg}, 72 \%)$. Known compound ${ }^{[2]} .{ }^{1} \mathrm{H}$ NMR $(400 \mathrm{MHz}$, Chloroform- $d$ ) $\delta 7.68-7.63(\mathrm{~m}, 2 \mathrm{H})$, $7.22-7.18(\mathrm{~m}, 5 \mathrm{H}), 7.02(\mathrm{t}, J=8.5 \mathrm{~Hz}, 2 \mathrm{H}), 5.27(\mathrm{t}, J=7.0 \mathrm{~Hz}, 1 \mathrm{H}), 3.90(\mathrm{dd}, J=14.9,6.8 \mathrm{~Hz}, 1 \mathrm{H})$, $3.80(\mathrm{dd}, J=14.8,7.1 \mathrm{~Hz}, 1 \mathrm{H}) .{ }^{19} \mathrm{~F}$ NMR $\left(376 \mathrm{MHz}\right.$, Chloroform- $d$ ) $\delta-102.99 .{ }^{13} \mathrm{C}$ NMR $(101 \mathrm{MHz}$, Chloroform- $d$ ) $\delta 165.8(\mathrm{~d}, J=257.0 \mathrm{~Hz}$ ), 138.3, $135.2(\mathrm{~d}, J=3.2 \mathrm{~Hz}), 131.1$ (d, $J=9.7 \mathrm{~Hz}), 129.3,129.0$, 127.2, $116.4(\mathrm{~d}, J=22.7 \mathrm{~Hz}), 64.2,55.1$. HRMS (ESI/Q-TOF) $\mathrm{m} / \mathrm{z}$ : $[\mathrm{M}+\mathrm{Na}]^{+}$calcd for $\mathrm{C}_{14} \mathrm{H}_{12} \mathrm{ClFNaO}_{2} \mathrm{~S}$ 321.0123; found: 321.0130 .<smiles>O=S(=O)(CC(Cl)c1ccccc1)c1ccc(Cl)cc1</smiles>

\section{(S)-1-chloro-4-((2-chloro-2-phenylethyl)sulfonyl)benzene (4I) :}

Prepared by the general procedure at the $60{ }^{\circ} \mathrm{C}$; isolated as white solid using petroleum/ethyl acetate $(10: 1)$ as eluent $(36.7 \mathrm{mg}, 58 \%)$.

Known compound ${ }^{[4]}{ }^{1} \mathrm{H}$ NMR (400 MHz, Chloroform-d) $\delta 7.64$ (d, J = $8.4 \mathrm{~Hz}, 2 \mathrm{H}), 7.39$ (d, J = 8.4 Hz, 2H), $7.31-7.22(\mathrm{~m}, 5 \mathrm{H}), 5.33$ (t, J = 7.0 Hz, 1H), 3.97 (dd, J = 14.9, $6.8 \mathrm{~Hz}, 1 \mathrm{H}), 3.87$ (dd, J = 14.9, 7.1 Hz, 1H). ${ }^{13} \mathrm{C}$ NMR (101 MHz, Chloroform- $d$ ) $\delta 140.6,138.2,137.6$, 129.7, 129.4, 129.3, 129.0, 127.2, 64.2, 55.0. HRMS (ESI/Q-TOF) $\mathrm{m} / \mathrm{z}:[\mathrm{M}+\mathrm{Na}]^{+}$calcd for $\mathrm{C}_{14} \mathrm{H}_{12} \mathrm{Cl}_{2} \mathrm{NaO}_{2} \mathrm{~S} 336.9833$; found: 336.9832 .<smiles>O=S(=O)(CC(Cl)c1ccccc1)c1cccc(I)c1</smiles>

(S)-1-((2-chloro-2-phenylethyl)sulfonyl)-3-iodobenzene (4m) : Prepared by the general procedure at the $60{ }^{\circ} \mathrm{C}$; isolated as white solid using petroleum/ethyl acetate (10:1) as eluent $(49.2 \mathrm{mg}, 60 \%){ }^{1} \mathrm{H} \mathrm{NMR}(400 \mathrm{MHz}$, Chloroform- $d$ ) $\delta 7.85(\mathrm{~s}, 1 \mathrm{H}), 7.79(\mathrm{~d}, J=8.0 \mathrm{~Hz}, 1 \mathrm{H}), 7.62(\mathrm{~d}, J=8.0 \mathrm{~Hz}$, 
1H), $7.30-7.13(\mathrm{~m}, 5 \mathrm{H}), 7.09$ (t, $J=8.0 \mathrm{~Hz}, 1 \mathrm{H}), 5.27$ (t, $J=8.0 \mathrm{~Hz}, 1 \mathrm{H}), 3.91$ (dd, $J=14.8,6.4 \mathrm{~Hz}$, 1H), 3.83 (dd, $J=14.8,7.6 \mathrm{~Hz}, 1 \mathrm{H}) .{ }^{13} \mathrm{C}$ NMR (101 MHz, Chloroform- $d$ ) $\delta 142.7,140.8,137.8,136.8$, 130.6, 129.6, 128.9, 127.2, 127.1, 94.2, 64.0, 55.0. HRMS (ESI/Q-TOF) m/z: $[\mathrm{M}+\mathrm{Na}]^{+}$calcd for $\mathrm{C}_{14} \mathrm{H}_{12} \mathrm{ClINaO}_{2} \mathrm{~S} 428.9189$; found: 428.9186 .<smiles>CS(=O)(=O)c1ccc(S(=O)(=O)CC(Cl)c2ccccc2)cc1</smiles>

(S)-1-((2-chloro-2-phenylethyl)sulfonyl)-4(methylsulfonyl)benzene (4n) : Prepared by the general procedure at the $60{ }^{\circ} \mathrm{C}$; isolated as white solid using petroleum/ethyl acetate (10:1) as eluent $(40.2 \mathrm{mg}, 56 \%) .{ }^{1} \mathrm{H}$ NMR (400 MHz, Chloroform- $d$ ) $\delta 7.97$ (d, $J=8.4 \mathrm{~Hz}, 2 \mathrm{H}), 7.88$ (d, $J=$ $8.4 \mathrm{~Hz}, 2 \mathrm{H}), 7.26-7.20(\mathrm{~m}, 5 \mathrm{H}), 5.36(\mathrm{t}, J=7.1 \mathrm{~Hz}, 1 \mathrm{H}), 4.03(\mathrm{dd}, J=15.0,6.7 \mathrm{~Hz}, 1 \mathrm{H}), 3.94(\mathrm{dd}, J=$ 15.0, 7.5 Hz, 1H), 3.07 (s, 3H). ${ }^{13} \mathrm{C}$ NMR (101 MHz, Chloroform- $d$ ) $\delta 145.2,144.2,137.7,129.5,129.3$, 129.1, 128.2, 127.3, 64.0, 54.8, 44.3. HRMS (ESI/Q-TOF) m/z: $[\mathrm{M}+\mathrm{Na}]^{+}$calcd for $\mathrm{C}_{15} \mathrm{H}_{15} \mathrm{ClNaO}_{4} \mathrm{~S}_{2}$ 380.9998; found: 381.0000 .<smiles>O=S(=O)(CC(Cl)c1ccccc1)c1ccc(C(F)(F)F)cc1</smiles>

(S)-1-((2-chloro-2-phenylethyl)sulfonyl)-4(trifluoromethyl)benzene (40) : Prepared by general procedure; isolated as a pale yellow solid using petroleum/ethyl acetate (10:1) as eluent $(29.8 \mathrm{mg}, 43 \%)$. Known compound ${ }^{[2]} .{ }^{1} \mathrm{H}$ NMR $(400 \mathrm{MHz}$, Chloroform- $d$ ) $\delta 7.83(\mathrm{~d}, J=8.2 \mathrm{~Hz}, 2 \mathrm{H}), 7.69(\mathrm{~d}, J=8.2 \mathrm{~Hz}, 2 \mathrm{H}), 7.33-7.17(\mathrm{~m}, 5 \mathrm{H}), 5.38(\mathrm{t}, J=7.0$ $\mathrm{Hz}, 1 \mathrm{H}), 4.04(\mathrm{dd}, J=15.0,6.6 \mathrm{~Hz}, 1 \mathrm{H}), 3.95(\mathrm{dd}, J=15.0,7.5 \mathrm{~Hz}, 1 \mathrm{H}) .{ }^{19} \mathrm{~F}$ NMR $(376 \mathrm{MHz}$, Chloroform- $d$ ) $\delta-63.37 .{ }^{13} \mathrm{C}$ NMR (101 MHz, Chloroform- $d$ ) $\delta 142.6,137.8,135.3$ (q, $J=33.4 \mathrm{~Hz}$ ), 129.4, 129.0, 128.8, 127.2, 126.2 (q, $J=3.7 \mathrm{~Hz}), 123.0$ (q, $J=273.1 \mathrm{~Hz}), 64.0,54.9$. HRMS (ESI/QTOF) m/z: $[\mathrm{M}+\mathrm{Na}]^{+}$calcd for $\mathrm{C}_{15} \mathrm{H}_{12} \mathrm{ClF}_{3} \mathrm{NaO}_{2} \mathrm{~S}$ 371.0096; found: 371.0098 .<smiles>N#Cc1ccc(S(=O)(=O)CC(Cl)c2ccccc2)cc1</smiles>

(S)-4-((2-chloro-2-phenylethyl)sulfonyl)benzonitrile (4p) : Prepared by the general procedure at the $60{ }^{\circ} \mathrm{C}$; isolated as white solid using petroleum/ethyl acetate (10:1) as eluent (31 mg, 50\%). Known compound ${ }^{[3]}{ }^{1} \mathrm{H}$ NMR $(400 \mathrm{MHz}$, Chloroform- $d$ ) $\delta 7.74(\mathrm{~d}, J=8.6 \mathrm{~Hz}$, 2H), $7.63(\mathrm{~d}, J=8.6 \mathrm{~Hz}, 2 \mathrm{H}), 7.23-7.13(\mathrm{~m}, 5 \mathrm{H}), 5.28(\mathrm{t}, J=7.0 \mathrm{~Hz}, 1 \mathrm{H}), 3.95(\mathrm{dd}, J=15.0,6.8 \mathrm{~Hz}$, $1 \mathrm{H}), 3.84$ (dd, $J=15.0,7.2 \mathrm{~Hz}, 1 \mathrm{H}) .{ }^{13} \mathrm{C}$ NMR (101 MHz, Chloroform-d) $\delta 143.3,137.8,132.7,129.5$, $129.1,128.9,127.2,117.4,117.0,64.0,54.9$. HRMS (ESI/Q-TOF) $\mathrm{m} / \mathrm{z}:[\mathrm{M}+\mathrm{Na}]^{+}$calcd for $\mathrm{C}_{15} \mathrm{H}_{12} \mathrm{ClNNaO}_{2} \mathrm{~S} 328.0169$; found: 328.0170 .<smiles>CC(C)[C@@H]1CC[C@@H](C)[C@H](OC(=O)c2ccc(S(=O)(=O)CC(Cl)c3ccc(F)cc3)cc2)C1</smiles>

(2S,5S)-5-isopropyl-2-methylcyclohexyl 4-(((R)-2chloro-2-(4-fluorophenyl)ethyl)sulfonyl)benzoate (4q) : Prepared by the general procedure at the $60^{\circ} \mathrm{C}$; isolated as white solid using petroleum/ethyl acetate (5:1) as eluent (71.1 mg, 74\%). ${ }^{1} \mathrm{H}$ NMR $(400 \mathrm{MHz}$, Chloroform- $d$ ) $\delta 8.02(\mathrm{~d}, J=8.1 \mathrm{~Hz}, 2 \mathrm{H}), 7.72(\mathrm{dd}, J$ $=8.4,6.4 \mathrm{~Hz}, 2 \mathrm{H}), 7.20-7.14(\mathrm{~m}, 2 \mathrm{H}), 6.85(\mathrm{td}, J=$ $8.5,1.7 \mathrm{~Hz}, 2 \mathrm{H}), 5.27$ (t, $J=7.0 \mathrm{~Hz}, 1 \mathrm{H}), 4.90$ (tdd, $J=10.9,4.4,1.5 \mathrm{~Hz}, 1 \mathrm{H}), 3.90$ (dd, $J=14.8,6.6$ $\mathrm{Hz}, 1 \mathrm{H}), 3.80$ (ddd, $J=14.8,7.5,2.4 \mathrm{~Hz}, 1 \mathrm{H}), 2.08-2.00(\mathrm{~m}, 1 \mathrm{H}), 1.89-1.79(\mathrm{~m}, 1 \mathrm{H}), 1.71-1.63(\mathrm{~m}$, 2H), $1.55-1.44(\mathrm{~m}, 2 \mathrm{H}), 1.12-1.00(\mathrm{~m}, 2 \mathrm{H}), 0.92-0.79(\mathrm{~m}, 7 \mathrm{H}), 0.73(\mathrm{dd}, J=7.0,1.3 \mathrm{~Hz}, 3 \mathrm{H}) .{ }^{19} \mathrm{~F}$ 
NMR (376 MHz, Chloroform- $d$ ) $\delta-111.23 .{ }^{13} \mathrm{C}$ NMR (101 MHz, Chloroform- $d$ ) $\delta 164.3,162.9(\mathrm{~d}, J=$ $249.9 \mathrm{~Hz}), 142.7$ (d, $J=2.9 \mathrm{~Hz}), 135.7$ (d, $J=5.2 \mathrm{~Hz}), 134.1$ (t, $J=3.4 \mathrm{~Hz}), 130.2$ (d, $J=1.4 \mathrm{~Hz}), 129.2$ $(\mathrm{dd}, J=8.6,2.8 \mathrm{~Hz}), 128.2(\mathrm{~d}, J=2.2 \mathrm{~Hz}), 116.0(\mathrm{dd}, J=22.0,1.6 \mathrm{~Hz}), 76.1,64.0,54.2,47.2,40.8,34.2$, 31.5, 26.6, 23.6, 22.0, 20.7, 16.5. HRMS (ESI/Q-TOF) $\mathrm{m} / \mathrm{z}:[\mathrm{M}+\mathrm{Na}]^{+}$calcd for $\mathrm{C}_{25} \mathrm{H}_{30} \mathrm{ClFNaO}_{4} \mathrm{~S}$ 503.1430; found: 503.1432 .<smiles>COc1ccc(S(=O)(=O)CC(Cl)c2ccc(-c3ccccc3)cc2)cc1</smiles>

(S)-4-(1-chloro-2-((4-methoxyphenyl)sulfonyl)ethyl)1,1'-biphenyl (4r) : Prepared by the general procedure at the $60{ }^{\circ} \mathrm{C}$; isolated as white solid using petroleum/ethyl acetate (10:1) as eluent (74 mg, 95\%). ${ }^{1} \mathrm{H}$ NMR (400 MHz, Chloroform- $d$ ) $\delta 7.52(\mathrm{~d}, J=8.9 \mathrm{~Hz}, 2 \mathrm{H}), 7.44(\mathrm{~d}, J=7.1$ $\mathrm{Hz}, 2 \mathrm{H}), 7.39-7.32$ (m, 4H), 7.28 (d, J=7.3 Hz, 1H), $7.21(\mathrm{~d}, J=8.4 \mathrm{~Hz}, 2 \mathrm{H}), 6.73(\mathrm{~d}, J=8.9 \mathrm{~Hz}, 2 \mathrm{H})$, $5.29(\mathrm{t}, J=7.0 \mathrm{~Hz}, 1 \mathrm{H}), 3.96-3.79(\mathrm{~m}, 2 \mathrm{H}), 3.64(\mathrm{~s}, 3 \mathrm{H}) .{ }^{13} \mathrm{C}$ NMR $(101 \mathrm{MHz}$, Chloroform- $d) \delta 163.8$, $142.1,140.1,137.2,130.5,130.4,128.9,127.8,127.8,127.5,127.1,114.3,64.1,55.6,55.1$. HRMS (ESI/Q-TOF) m/z: [M+Na] ${ }^{+}$calcd for $\mathrm{C}_{16} \mathrm{H}_{21} \mathrm{NNaO}_{3} \mathrm{~S} 409.0636$; found: 409.0637.<smiles>COc1ccc(S(=O)(=O)CC(Cl)c2ccc3ccccc3c2)cc1</smiles>

(S)-2-(1-chloro-2-((4methoxyphenyl)sulfonyl)ethyl)naphthalene (4s) : Prepared by the general procedure at the $60{ }^{\circ} \mathrm{C}$; isolated as white solid using petroleum/ethyl acetate (10:1) as eluent (71 mg, 98\%).

${ }^{1} \mathrm{H}$ NMR (400 MHz, Chloroform- $d$ ) $\delta 7.70-7.63(\mathrm{~m}, 2 \mathrm{H}), 7.62-7.55(\mathrm{~m}, 2 \mathrm{H}), 7.46-7.36(\mathrm{~m}, 4 \mathrm{H})$, $7.23-7.16(\mathrm{~m}, 1 \mathrm{H}), 6.53(\mathrm{~d}, J=9.0 \mathrm{~Hz}, 2 \mathrm{H}), 5.40(\mathrm{t}, J=7.0 \mathrm{~Hz}, 1 \mathrm{H}), 3.99-3.84(\mathrm{~m}, 2 \mathrm{H}), 3.56(\mathrm{~s}, 3 \mathrm{H})$. ${ }^{13} \mathrm{C}$ NMR (101 MHz, Chloroform- $d$ ) $\delta$ 163.6, 135.3, 133.4, 132.8, 130.3, 130.2, 129.1, 128.1, 127.6, 127.0, 126.9, 126.7, 124.0, 114.0, 64.0, 55.7, 55.5. HRMS (ESI/Q-TOF) m/z: $[\mathrm{M}+\mathrm{Na}]^{+}$calcd for $\mathrm{C}_{19} \mathrm{H}_{17} \mathrm{ClNaO}_{3} \mathrm{~S} 383.0479$; found: 383.0480 .

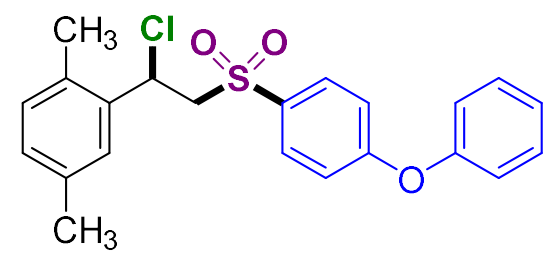

(R)-2-(1-chloro-2-((4-phenoxyphenyl)sulfonyl)ethyl)-1,4dimethylbenzene (4t) : Prepared by the general procedure at the $60{ }^{\circ} \mathrm{C}$; isolated as white solid using petroleum/ethyl acetate (10:1) as eluent (75.4 mg, 94\%). ${ }^{1} \mathrm{H}$ NMR (400 MHz, Chloroform- $d$ ) $\delta 7.51(\mathrm{~d}, J=8.9 \mathrm{~Hz}, 2 \mathrm{H}), 7.34(\mathrm{t}, J=7.9 \mathrm{~Hz}$, $2 \mathrm{H}), 7.18-7.13(\mathrm{~m}, 1 \mathrm{H}), 6.98-6.93(\mathrm{~m}, 2 \mathrm{H}), 6.92-6.85(\mathrm{~m}, 2 \mathrm{H}), 6.84-6.78(\mathrm{~m}, 3 \mathrm{H}), 5.52(\mathrm{t}, J=7.0$ $\mathrm{Hz}, 1 \mathrm{H}), 3.93-3.80$ (m, 2H), 2.29 (s, 3H), 2.10 (s, 3H). ${ }^{13} \mathrm{C}$ NMR (101 MHz, Chloroform-d) $\delta ~ 162.5$, 154.8, 136.2, 136.0, 132.6, 132.4, 130.9, 130.33, 130.29, 129.8, 127.3, 125.3, 120.5, 117.3, 63.3, 51.4, 20.9, 18.7. HRMS (ESI/Q-TOF) m/z: $[\mathrm{M}+\mathrm{Na}]^{+}$calcd for $\mathrm{C}_{22} \mathrm{H}_{21} \mathrm{ClNaO}_{3} \mathrm{~S}$ 423.0792; found: 423.0793.<smiles>COc1ccc(S(=O)(=O)CC(Cl)c2ccc(C(C)(C)C)cc2)cc1</smiles>

(R)-1-(tert-butyl)-4-(1-chloro-2-((4methoxyphenyl)sulfonyl)ethyl)benzene (4u) : Prepared by the general procedure at the $60^{\circ} \mathrm{C}$; isolated as white solid using petroleum/ethyl acetate $(10: 1)$ as eluent $(67.5 \mathrm{mg}, 92 \%) .{ }^{1} \mathrm{H}$ 
NMR (400 MHz, Chloroform- $d$ ) $\delta 7.55(\mathrm{~d}, J=8.9 \mathrm{~Hz}, 2 \mathrm{H}), 7.18(\mathrm{~d}, J=8.3 \mathrm{~Hz}, 2 \mathrm{H}), 7.09$ (d, $J=8.4 \mathrm{~Hz}$, 2H), 6.78 (d, $J=8.9 \mathrm{~Hz}, 2 \mathrm{H}), 5.24$ (t, $J=6.9 \mathrm{~Hz}, 1 \mathrm{H}), 3.87(\mathrm{dd}, J=14.8,6.7 \mathrm{~Hz}, 1 \mathrm{H}), 3.83-3.73(\mathrm{~m}$, 4H), 1.20 (s, 9H). ${ }^{13} \mathrm{C}$ NMR (151 MHz, Chloroform- $d$ ) $\delta 163.7,152.3,135.4,130.6,130.4,126.8,125.8$, 114.2, 64.2, 55.6, 55.2, 34.6, 31.2. HRMS (ESI/Q-TOF) m/z: $[\mathrm{M}+\mathrm{Na}]^{+}$calcd for $\mathrm{C}_{19} \mathrm{H}_{23} \mathrm{ClNaO}_{3} \mathrm{~S}$ 389.0949; found: 389.0947 .

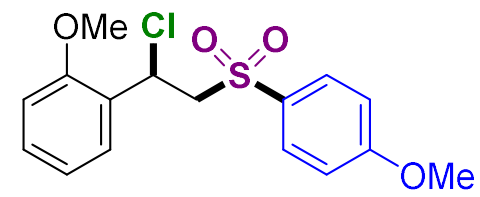

(S)-1-(1-chloro-2-((4-methoxyphenyl)sulfonyl)ethyl)-2methoxybenzene (4v) : Prepared by the general procedure at the $60{ }^{\circ} \mathrm{C}$; isolated as white solid using petroleum/ethyl acetate $(10: 1)$ as eluent (64 mg, 94\%). 1H NMR (400 MHz, Chloroform-d) $\delta 7.59$ (d, $\mathrm{J}=9.0 \mathrm{~Hz}, 2 \mathrm{H}), 7.20-7.12(\mathrm{~m}, 2 \mathrm{H}), 6.82-6.77(\mathrm{~m}, 3 \mathrm{H}), 6.66(\mathrm{dd}, \mathrm{J}=8.3,1.1 \mathrm{~Hz}, 1 \mathrm{H}), 5.54(\mathrm{t}, \mathrm{J}=6.9$ $\mathrm{Hz}, 1 \mathrm{H}), 3.97(\mathrm{dd}, \mathrm{J}=14.8,6.7 \mathrm{~Hz}, 1 \mathrm{H}), 3.81(\mathrm{dd}, \mathrm{J}=7.1 \mathrm{~Hz}, 1 \mathrm{H}), 3.76(\mathrm{~s}, 3 \mathrm{H}), 3.68(\mathrm{~s}, 3 \mathrm{H}) .{ }^{13} \mathrm{C} \mathrm{NMR}$ (101 MHz, Chloroform- $d$ ) $\delta 163.7,156.3,130.6,130.4,128.8,126.2,120.8,114.1,111.1,62.7,55.7$, 55.5, 51.2. HRMS (ESI/Q-TOF) m/z: [M+Na] calcd for $\mathrm{C}_{16} \mathrm{H}_{17} \mathrm{ClNaO}_{4} \mathrm{~S}$ 363.0428; found: 363.0421 .<smiles>COc1ccc(S(=O)(=O)CC(Cl)c2ccc(OC(F)(F)F)cc2)cc1</smiles>

(S)-1-(1-chloro-2-((4-methoxyphenyl)sulfonyl)ethyl)-4(trifluoromethyl)benzene (4w) : Prepared by the general procedure at the $60{ }^{\circ} \mathrm{C}$; isolated as white solid using petroleum/ethyl acetate $(10: 1)$ as eluent $(56.6 \mathrm{mg}, 78 \%) .{ }^{1} \mathrm{H}$ NMR (400 MHz, Chloroform- $d$ ) $\delta 7.61(\mathrm{~d}, J=8.9 \mathrm{~Hz}, 2 \mathrm{H}), 7.31(\mathrm{~d}, J=8.7 \mathrm{~Hz}, 2 \mathrm{H}), 7.10(\mathrm{~d}, J=8.1 \mathrm{~Hz}$, 2H), $6.88(\mathrm{~d}, J=8.9 \mathrm{~Hz}, 2 \mathrm{H}), 5.34$ (dd, $J=7.9,6.2 \mathrm{~Hz}, 1 \mathrm{H}), 3.93(\mathrm{dd}, J=14.7,6.3 \mathrm{~Hz}, 1 \mathrm{H}), 3.88-3.80$ $(\mathrm{m}, 4 \mathrm{H}) .{ }^{19} \mathrm{~F}$ NMR $\left(376 \mathrm{MHz}\right.$, Chloroform- $d$ ) $\delta-57.85 .{ }^{13} \mathrm{C}$ NMR $(101 \mathrm{MHz}$, Chloroform- $d$ ) $\delta$ 164.0, 149.4 (q, $J=1.7 \mathrm{~Hz}$ ), 137.1, 130.5, 130.3, 128.9, 121.1, 120.3 (q, $J=258.0 \mathrm{~Hz}$ ), 114.4, 64.1, 55.6, 54.3. HRMS (ESI/Q-TOF) m/z: [M+Na] $]^{+}$calcd for $\mathrm{C}_{16} \mathrm{H}_{14} \mathrm{ClF}_{3} \mathrm{NaO}_{4} \mathrm{~S}$ 417.0151. found: 417.0147 .<smiles>COc1ccc(S(=O)(=O)CC(Cl)c2ccc(F)cc2)cc1</smiles>

(S)-1-(1-chloro-2-((4-methoxyphenyl)sulfonyl)ethyl)-4fluorobenzene (4x): Prepared by the general procedure at the $60{ }^{\circ} \mathrm{C}$; isolated as white solid using petroleum/ethyl acetate $(10: 1)$ as eluent (62 mg, 94\%). ${ }^{1} \mathrm{H}$ NMR (400 MHz, Chloroform- $d$ ) $\delta$ $7.65(\mathrm{~d}, J=8.9 \mathrm{~Hz}, 2 \mathrm{H}), 7.30-7.23(\mathrm{~m}, 2 \mathrm{H}), 7.00-6.90(\mathrm{~m}, 4 \mathrm{H}), 5.33(\mathrm{t}, J=7.0 \mathrm{~Hz}, 1 \mathrm{H}), 3.98-3.80$ $(\mathrm{m}, 5 \mathrm{H}) .{ }^{13} \mathrm{C}$ NMR $(101 \mathrm{MHz}$, Chloroform- $d) \delta 163.9,162.8(\mathrm{~d}, J=249.1 \mathrm{~Hz}), 134.5(\mathrm{~d}, J=3.4 \mathrm{~Hz})$, $130.5,130.3,129.2$ (d, $J=8.5 \mathrm{~Hz}), 115.9$ (d, $J=21.8 \mathrm{~Hz}), 114.4,64.2,55.8,54.5$. HRMS (ESI/Q-TOF) $\mathrm{m} / \mathrm{z}$ : $[\mathrm{M}+\mathrm{Na}]^{+}$calcd for $\mathrm{C}_{15} \mathrm{H}_{14} \mathrm{ClO}_{3} \mathrm{SNa} 351.0228$; found: 351.0228 . HRMS (ESI/Q-TOF) m/z: [M+Na] calcd for $\mathrm{C}_{15} \mathrm{H}_{14} \mathrm{ClFO}_{3} \mathrm{SNa} 351.0228$; found: 351.0228 .<smiles>COc1ccc(S(=O)(=O)CC(Cl)c2ccc(Cl)cc2)cc1</smiles>

(R)-1-chloro-4-(1-chloro-2-((4methoxyphenyl)sulfonyl)ethyl)benzene (4y) : Prepared by the general procedure at the $60{ }^{\circ} \mathrm{C}$; isolated as white solid using petroleum/ethyl acetate (10:1) as eluent (62 mg, 98\%). ${ }^{1} \mathrm{H}$ NMR

(400 MHz, Chloroform- $d$ ) $\delta 7.61(\mathrm{~d}, J=8.9 \mathrm{~Hz}, 2 \mathrm{H}), 7.26-7.18(\mathrm{~m}, 4 \mathrm{H}), 6.89$ (d, $J=8.9 \mathrm{~Hz}, 2 \mathrm{H}), 5.31$ $(\mathrm{dd}, J=7.9,6.2 \mathrm{~Hz}, 1 \mathrm{H}), 3.93(\mathrm{dd}, J=14.7,6.3 \mathrm{~Hz}, 1 \mathrm{H}), 3.89-3.81(\mathrm{~m}, 4 \mathrm{H}) .{ }^{13} \mathrm{C}$ NMR $(101 \mathrm{MHz}$, 
Chloroform- $d$ ) $\delta 163.9,136.9,135.0,130.4,130.3,129.0,128.7,114.4,64.0,55.7,54.5$. HRMS (ESI/QTOF) m/z: [M+Na] calcd for $\mathrm{C}_{15} \mathrm{H}_{14} \mathrm{Cl}_{2} \mathrm{NaO}_{3} \mathrm{~S} 366.9933$; found: 366.9935 .

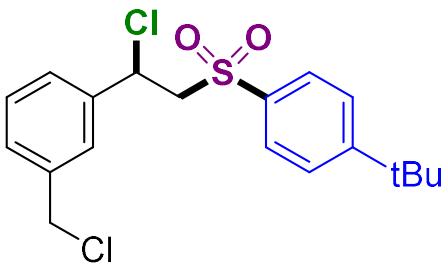

(R)-1-(2-((4-(tert-butyl)phenyl)sulfonyl)-1-chloroethyl)-3(chloromethyl)benzene (4z) : Prepared by the general procedure at the $60{ }^{\circ} \mathrm{C}$; isolated as white solid using petroleum/ethyl acetate $(10: 1)$ as eluent (62 mg, 64\%). ${ }^{1} \mathrm{H}$ NMR (400 MHz, Chloroform- $d$ ) $\delta 7.54(\mathrm{~d}$, $J=8.6 \mathrm{~Hz}, 2 \mathrm{H}), 7.37$ (d, $J=8.6 \mathrm{~Hz}, 2 \mathrm{H}), 7.20$ (s, 4H), 5.29 (dd, $J=$ 7.7, $6.4 \mathrm{~Hz}, 1 \mathrm{H}), 4.45(\mathrm{dd}, J=1.3 \mathrm{~Hz}, 2 \mathrm{H}), 3.87$ (dd, $J=14.7,6.4 \mathrm{~Hz}$, 1H), $3.80(\mathrm{dd}, J=14.7,7.7 \mathrm{~Hz}, 1 \mathrm{H}), 1.25$ (s, 9H). ${ }^{13} \mathrm{C}$ NMR (101 MHz, Chloroform- $d$ ) $\delta 157.9,138.6$, 138.4, 136.1 129.0, 127.9, 127.7, 126.2, 63.9, 54.6, 45.4, 35.3, 31.0. HRMS (ESI/Q-TOF) m/z: [M+Na] ${ }^{+}$ calcd for $\mathrm{C}_{19} \mathrm{H}_{22} \mathrm{Cl}_{2} \mathrm{NaO}_{2} \mathrm{~S} 407.0610$; found: 407.0611 .<smiles>COc1ccc(S(=O)(=O)CC(Cl)c2ccccc2Br)cc1</smiles>

(S)-1-bromo-2-(1-chloro-2-((4methoxyphenyl)sulfonyl)ethyl)benzene (4aa): Prepared by the general procedure at the $60{ }^{\circ} \mathrm{C}$; isolated as white solid using petroleum/ethyl acetate (10:1) as eluent (57 mg, 65\%). ${ }^{1} \mathrm{H}$ NMR (400 MHz, Chloroform- $d$ ) $\delta 7.69$ (d, $J=8.9 \mathrm{~Hz}, 2 \mathrm{H}), 7.43$ (d, $J=8.0 \mathrm{~Hz}, 1 \mathrm{H}), 7.33(\mathrm{~d}, J=7.6 \mathrm{~Hz}, 1 \mathrm{H}), 7.18$ (td, $J=7.7,6.9,1.8 \mathrm{~Hz}, 1 \mathrm{H}), 7.06(\mathrm{td}, J=7.7,1.7 \mathrm{~Hz}, 1 \mathrm{H}), 6.86(\mathrm{~d}, J=8.9 \mathrm{~Hz}, 2 \mathrm{H}), 5.67$ (t, $J=7.0 \mathrm{~Hz}$, $1 \mathrm{H}), 3.86-3.67$ (m, 5H). ${ }^{13} \mathrm{C}$ NMR (101 MHz, Chloroform- $d$ ) $\delta 164.0,137.5,133.3,130.6,130.4,130.3$, 128.9, 128.2, 122.9, 114.5, 63.0, 55.8, 53.9. HRMS (ESI/Q-TOF) $\mathrm{m} / \mathrm{z}:[\mathrm{M}+\mathrm{Na}]^{+}$calcd for $\mathrm{C}_{15} \mathrm{H}_{14} \mathrm{BrClNaO}_{3} \mathrm{~S}$ 410.9428; found: 410.9414.<smiles>COc1ccc(S(=O)(=O)CC(Cl)c2ccc(C#N)cc2)cc1</smiles>

(S)-4-(1-chloro-2-((4methoxyphenyl)sulfonyl)ethyl)benzonitrile (4ab) : Prepared by the general procedure at the $60{ }^{\circ} \mathrm{C}$; isolated as white solid using petroleum/ethyl acetate (10:1) as eluent (45 mg, 67\%). ${ }^{1} \mathrm{H}$ NMR (400 MHz, Chloroform- $d$ ) $\delta 7.64$ (d, $\left.J=8.9 \mathrm{~Hz}, 2 \mathrm{H}\right), 7.58$ (d, $\left.J=8.4 \mathrm{~Hz}, 2 \mathrm{H}\right), 7.42$ $(\mathrm{d}, J=8.4 \mathrm{~Hz}, 2 \mathrm{H}), 6.91(\mathrm{~d}, J=8.9 \mathrm{~Hz}, 2 \mathrm{H}), 5.34(\mathrm{t}, J=7.6 \mathrm{~Hz}, 1 \mathrm{H}), 3.95-3.76(\mathrm{~m}, 5 \mathrm{H}) .{ }^{13} \mathrm{C} \mathrm{NMR}$ (101 MHz, Chloroform-d) $\delta 164.1,143.4,132.7,130.31,130.25,128.2,118.0,114.5,113.0,63.7,55.8$, 54.0. HRMS (ESI/Q-TOF) m/z: [M+Na] ${ }^{+}$calcd for $\mathrm{C}_{16} \mathrm{H}_{14} \mathrm{ClNNaO}_{3} \mathrm{~S}$ 358.0275; found: 358.0275 .<smiles>CC(=O)c1ccc(C(Cl)CS(=O)(=O)c2ccc(Oc3ccccc3)cc2)cc1</smiles>

methyl

(S)-4-(1-chloro-2-((4 phenoxyphenyl)sulfonyl)ethyl)benzoate (4ac) : Prepared by the general procedure at the $60{ }^{\circ} \mathrm{C}$; isolated as white solid using petroleum/ethyl acetate (10:1) as eluent (71mg, 82\%). ${ }^{1} \mathrm{H}$ NMR $(400 \mathrm{MHz}$, Chloroform- $d$ ) $\delta 7.87$ (d, $J=8.4 \mathrm{~Hz}, 2 \mathrm{H}), 7.56(\mathrm{~d}, J$ $=8.9 \mathrm{~Hz}, 2 \mathrm{H}), 7.37-7.31(\mathrm{~m}, 2 \mathrm{H}), 7.28(\mathrm{~d}, J=8.4 \mathrm{~Hz}, 2 \mathrm{H}), 7.19-7.13(\mathrm{~m}, 1 \mathrm{H}), 7.01-6.93(\mathrm{~m}, 2 \mathrm{H})$, $6.83(\mathrm{~d}, J=8.8 \mathrm{~Hz}, 2 \mathrm{H}), 5.29(\mathrm{~d}, J=6.8 \mathrm{~Hz}, 1 \mathrm{H}), 3.92-3.75(\mathrm{~m}, 5 \mathrm{H}) .{ }^{13} \mathrm{C}$ NMR $(101 \mathrm{MHz}$, Chloroformd) $\delta 166.2,162.8,154.6,143.1,132.1,130.8,130.5,130.3,130.2,127.4,125.4,120.5,117.3,63.8,54.4$, 52.3. HRMS (ESI/Q-TOF) m/z: [M+Na] $]^{+}$calcd for $\mathrm{C}_{22} \mathrm{H}_{19} \mathrm{ClNaO}_{5} \mathrm{~S} 453.0534$; found: 453.0536 . 
<smiles>COc1ccc(S(=O)(=O)CC(Cl)c2ccc(C(F)(F)F)cc2)cc1</smiles>

(S)-1-(1-chloro-2-((4-methoxyphenyl)sulfonyl)ethyl)-4(trifluoromethyl)benzene (4ad) : Prepared by the general procedure at the $60{ }^{\circ} \mathrm{C}$; isolated as white solid using petroleum/ethyl acetate (10:1) as eluent $(56.6 \mathrm{mg}, 78 \%) .{ }^{1} \mathrm{H}$ $2 \mathrm{H}), 6.85(\mathrm{~d}, J=8.9 \mathrm{~Hz}, 2 \mathrm{H}), 5.36(\mathrm{dd}, J=8.0,6.1 \mathrm{~Hz}, 1 \mathrm{H}), 3.94(\mathrm{dd}, J=14.8,6.1 \mathrm{~Hz}, 1 \mathrm{H}), 3.90-3.85$ $(\mathrm{m}, 1 \mathrm{H}), 3.84(\mathrm{~s}, 3 \mathrm{H}) .{ }^{19} \mathrm{~F}$ NMR $\left(376 \mathrm{MHz}, \mathrm{CDCl}_{3}\right) \delta-62.84 .{ }^{13} \mathrm{C}$ NMR $(101 \mathrm{MHz}$, Chloroform- $d) \delta$ 164.0, 149.4 (q, $J=7.6 \mathrm{~Hz}), 137.1,130.5,130.3,128.9,121.1,120.3$ (q, $J=258.0 \mathrm{~Hz}), 114.4,64.1,55.6$, 54.3. HRMS (ESI/Q-TOF) m/z: [M+Na] ${ }^{+}$calcd for $\mathrm{C}_{16} \mathrm{H}_{14} \mathrm{ClF}_{3} \mathrm{NaO}_{3} \mathrm{~S}$ 401.0202; found: 401.0200 .<smiles>O=c1ccc2ccc(C(Cl)CS(=O)(=O)c3ccc(Oc4ccccc4)cc3)cc2o1</smiles>

(S)-7-(1-chloro-2-((4-phenoxyphenyl)sulfonyl)ethyl)2H-chromen-2-one (4ae) : Prepared by the general procedure at the $60{ }^{\circ} \mathrm{C}$; isolated as white solid using petroleum/ethyl acetate (10:1) as eluent $(63 \mathrm{mg}, 72 \%) .{ }^{1} \mathrm{H}$ NMR (400 MHz, Chloroform- $d$ ) $\delta 7.64-7.56(\mathrm{~m}, 3 \mathrm{H}), 7.40-7.31(\mathrm{~m}, 3 \mathrm{H}), 7.24-7.14(\mathrm{~m}, 3 \mathrm{H}), 6.97$ (dd, $J=8.6,1.2 \mathrm{~Hz}, 2 \mathrm{H}), 6.87(\mathrm{~d}, J=8.9 \mathrm{~Hz}, 2 \mathrm{H}), 6.38(\mathrm{~d}, J=9.5 \mathrm{~Hz}, 1 \mathrm{H}), 5.30(\mathrm{~d}, J=7.0 \mathrm{~Hz}, 1 \mathrm{H})$, 3.93 - $3.74(\mathrm{~m}, 2 \mathrm{H}) .{ }^{13} \mathrm{C}$ NMR (101 MHz, Chloroform- $d$ ) $\delta 163.0,160.0,154.6,154.0,142.5,142.4$, 132.1, 130.5, 130.3, 128.4, 125.4, 123.5, 120.5, 119.3, 117.7, 117.4, 115.7, 63.8, 54.1. HRMS (ESI/QTOF) m/z: $[\mathrm{M}+\mathrm{Na}]^{+}$calcd for $\mathrm{C}_{22} \mathrm{H}_{17} \mathrm{ClNaO}_{5} \mathrm{~S}$ 463.0377; found: 463.0375 .

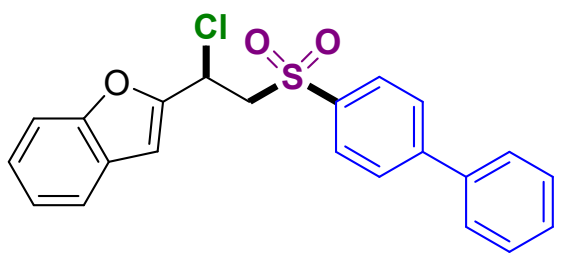

(S)-2-(2-([1,1'-biphenyl]-4-ylsulfonyl)-1chloroethyl)benzofuran (4af) : Prepared by the general procedure at the $60{ }^{\circ} \mathrm{C}$; isolated as white solid using petroleum/ethyl acetate (10:1) as eluent (60 mg, 76\%). ${ }^{1} \mathrm{H}$ NMR (400 MHz, Chloroform- $d$ ) $\delta 7.63(\mathrm{~d}, J=8.5 \mathrm{~Hz}, 2 \mathrm{H})$, $7.42-7.34(\mathrm{~m}, 1 \mathrm{H}), 7.37-7.27(\mathrm{~m}, 5 \mathrm{H}), 7.25(\mathrm{dd}, J=7.8,1.8 \mathrm{~Hz}, 2 \mathrm{H}), 7.12-7.01(\mathrm{~m}, 3 \mathrm{H}), 6.63(\mathrm{~s}$, $1 \mathrm{H}), 5.49(\mathrm{dd}, J=9.6,5.0 \mathrm{~Hz}, 1 \mathrm{H}), 4.26(\mathrm{dd}, J=14.7,9.6 \mathrm{~Hz}, 1 \mathrm{H}), 3.92(\mathrm{dd}, J=14.7,5.0 \mathrm{~Hz}, 1 \mathrm{H}) .{ }^{13} \mathrm{C}$ NMR (101 MHz, Chloroform-d) $\delta$ 154.8, 150.9, 146.7, 138.8, 136.7, 128.9, 128.7, 128.4, 127.4, 127.3, 127.1, 125.7, 123.3, 121.6, 111.3, 107.0, 60.9, 47.7. LCMS m/z: $[\mathrm{M}+\mathrm{Na}]^{+}$calcd for $\mathrm{C}_{22} \mathrm{H}_{17} \mathrm{ClNaO}_{3} \mathrm{~S}$ 419.05; found: 419.26 .<smiles>COC(=O)C(N)Cc1ccc(C(Cl)CS(=O)(=O)c2ccc(OC)cc2)cc1</smiles>

methoxyphenyl)sulfonyl)ethyl)phenyl)propanoate (4ag): Prepared by the general procedure at the $60{ }^{\circ} \mathrm{C}$; isolated as white solid using petroleum/ethyl acetate (5:1) as eluent $(53.2 \mathrm{mg}, 52 \%) .{ }^{1} \mathrm{H}$ NMR (400 MHz, Chloroform- $d$ ) $\delta 7.67$ (dd, $J=9.2,2.8 \mathrm{~Hz}, 2 \mathrm{H}), 7.20$ (d, $J=8.0 \mathrm{~Hz}, 2 \mathrm{H}), 7.04$ (d, $J=7.6 \mathrm{~Hz}$, 2H), $6.91(\mathrm{~d}, J=8.8 \mathrm{~Hz}, 2 \mathrm{H}), 5.28(\mathrm{t}, J=6.8 \mathrm{~Hz}, 1 \mathrm{H}), 4.99(\mathrm{~d}, J=8.0 \mathrm{~Hz}, 1 \mathrm{H}), 4.55(\mathrm{~d}, J=6.8 \mathrm{~Hz}, 1 \mathrm{H})$, 3.90 (ddd, $J=14.8,7.2,1.6 \mathrm{~Hz}, 1 \mathrm{H}), 3.85$ (s, 3H), 3.78 (dd, $J=14.8,6.4 \mathrm{~Hz}, 1 \mathrm{H}), 3.69$ (s, 3H), 3.13 $2.92(\mathrm{~m}, 2 \mathrm{H}), 1.39$ (s, 9H). ${ }^{13} \mathrm{C}$ NMR (101 MHz, Chloroform- $d$ ) $\delta$ 172.1, 163.9, 155.0, 137.3, 130.7, 
130.4, 129.88, 129.86, 127.3, 114.4, 80.1, 64.4, 55.7, 54.9, 54.3, 52.3, 38.0, 28.3. HRMS (ESI/Q-TOF) $\mathrm{m} / \mathrm{z}:[\mathrm{M}+\mathrm{Na}]^{+}$calcd for $\mathrm{C}_{24} \mathrm{H}_{30} \mathrm{ClNNaO}_{7} \mathrm{~S}$ 534.1324; found: 534.1322.

\section{Control experiments using other halide salts.}

In the glovebox, Styrenes ( $0.2 \mathrm{mmol}, 1.0$ equiv), aryldiazonium tetrafluoroborates $(0.3 \mathrm{mmol}, 1.5$ equiv), Cu1 (2.1 mg, $5.0 \mathrm{~mol} \%), \mathbf{L 1}(2.6 \mathrm{mg}, 5.0 \mathrm{~mol} \%$ ), organic salts containing halide (1.0 equiv) were added to chamber B, followed by addition of Me-THF (1.0 mL). Tetrabromothiophene S,Sdioxides $(0.6 \mathrm{mmol}, 259.0 \mathrm{mg})$ in tetradecane $(1.0 \mathrm{~mL})$ was added to chamber A, followed by addition of 4-methylphenylene $(0.5 \mathrm{mmol}, 62.9 \mu \mathrm{l})$. The two chamber system was sealed and removed out of the glovebox, then chamber $\mathrm{A}$ was heated to $100^{\circ} \mathrm{C}$ in heat block. About ten minutes later, the sulfur dioxide was completely released since the system in chamber A became clear. Then chamber B was heated to $60{ }^{\circ} \mathrm{C}$ in heat block. After 16 hours, two chamber was cooled to room temperature. The mixture in chamber B was passed through a short silica gel pad with ethyl acetate. Yield determined by ${ }^{1} \mathrm{H}$ NMR $\left(\mathrm{CDCl}_{3}\right)$ analysis using 1,2,3-trimethoxybenzene as the internal standard.

\section{Gram-scale reaction and transformations of $\beta$-chloroalkyl sulfones.}

\section{Gram-scale reaction}

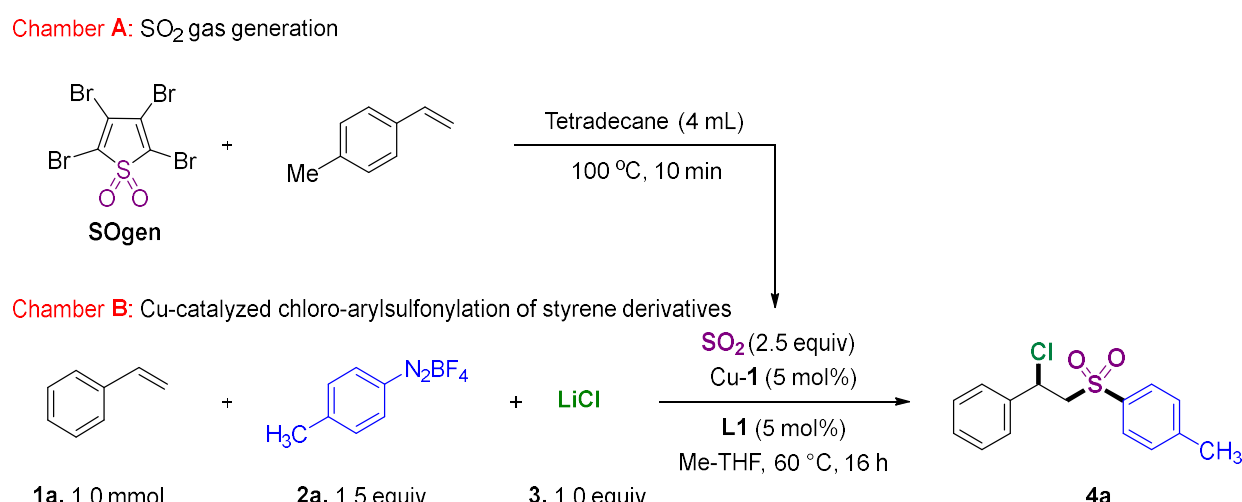

In the glovebox, Styrene (1.0 mmol, 1.0 equiv, $115.0 \mu \mathrm{l})$, Diazonium $p$-methyltetrafluoroborate (1.5 mmol, $308.9 \mathrm{mg})$, Cu1 (10.7 mg, $5.0 \mathrm{~mol} \%$ ), L1 (12.8 mg, $5.0 \mathrm{~mol} \%$ ), LiCl (42.4 mg, 1.0 equiv) were added to chamber B, followed by addition of Me-THF (4.0 mL). Tetrabromothiophene S,Sdioxides $(3.0 \mathrm{mmol}, 1295.2 \mathrm{mg})$ in tetradecane $(4.0 \mathrm{~mL})$ was added to chamber A, followed by addition of 4-methylphenylene $(2.5 \mathrm{mmol}, 287.5 \mu \mathrm{l})$. The two chamber system was sealed and removed out of the glovebox, then chamber A was heated to $100^{\circ} \mathrm{C}$ in heat block. About ten minutes later, the sulfur dioxide was completely released since the system in chamber A became clear. Then chamber B was heated to $60{ }^{\circ} \mathrm{C}$ in heat block. After 16 hours, two chamber was cooled to room temperature. The mixture in chamber B was passed through a short silica gel pad with ethyl acetate. The filtrate was washed by ethyl acetate and $\mathrm{H}_{2} \mathrm{O}(15 \mathrm{~mL} \times 3)$, dried by $\mathrm{Na}_{2} \mathrm{SO}_{4}$, then concentrated 
and the residue was purified by flash column chromatography to give $82 \%$ of $\mathbf{4 a}$.

\section{Transformations of $\beta$-chloroalkyl sulfones.}<smiles>Cc1ccc(S(=O)(=O)CC(Cl)c2ccccc2)cc1</smiles>

$4 a$

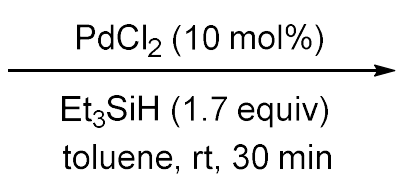

toluene, $\mathrm{rt}, 30 \mathrm{~min}$<smiles>Cc1ccc(S(=O)(=O)CCc2ccccc2)cc1</smiles>

7, $72 \%$

To a mixture of $4 \mathbf{a}(60.0 \mathrm{mg}, 0.2 \mathrm{mmol})$ in toluene $(1 \mathrm{~mL})$ was added, at $23{ }^{\circ} \mathrm{C}, \mathrm{Et}_{3} \mathrm{SiH}(39.2 \mathrm{mg}, 0.34$ mmol) and a catalytic amount of $\mathrm{PdCl}_{2}(3.5 \mathrm{mg})$. After stirring for $30 \mathrm{~min}$, toluene was distilled under vacuum, the crude product was recrystallized three times from hexanes, and the catalyst was removed by passing through an $\mathrm{Al}_{2} \mathrm{O}_{3}$ column to afford product $7(37.5 \mathrm{mg}, 72 \%)^{[5]} .{ }^{1} \mathrm{H} \mathrm{NMR}(400 \mathrm{MHz}$, Chloroformd) $\delta 7.74(\mathrm{~d}, J=8.4 \mathrm{~Hz}, 2 \mathrm{H}), 7.29$ (d, $J=8.4 \mathrm{~Hz}, 2 \mathrm{H}), 7.21-7.10(\mathrm{~m}, 3 \mathrm{H}), 7.04(\mathrm{~d}, J=7.0 \mathrm{~Hz}, 3 \mathrm{H}), 3.30$ $-3.23(\mathrm{~m}, 2 \mathrm{H}), 2.98-2.91(\mathrm{~m}, 2 \mathrm{H}), 2.38(\mathrm{~s}, 3 \mathrm{H}) .{ }^{13} \mathrm{C}$ NMR (101 MHz, Chloroform- $d$ ) $\delta$ 144.8, 137.6, 136.1, 130.0, 128.8, 128.3, 128.1, 126.9, 57.6, 28.8, 21.6. HRMS (ESI/Q-TOF) $\mathrm{m} / \mathrm{z}:[\mathrm{M}+\mathrm{Na}]^{+}$calcd for $\mathrm{C}_{15} \mathrm{H}_{16} \mathrm{NaO}_{2} \mathrm{~S} 283.0763$; found: 283.0762 .<smiles>Cc1ccc(S(=O)(=O)CC(Cl)c2ccccc2)cc1</smiles>

$4 a$

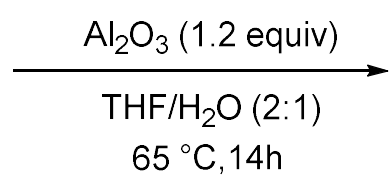

$65^{\circ} \mathrm{C}, 14 \mathrm{~h}$<smiles>Cc1ccc(S(=O)(=O)CC(O)c2ccccc2)cc1</smiles>

$8,41 \%$

To a solution of $4 \mathbf{a}(60.0 \mathrm{mg}, 0.2 \mathrm{mmol})$ in $\mathrm{THF} / \mathrm{H}_{2} \mathrm{O}(3.0 / 1.5 \mathrm{ml})$ was added $\mathrm{Al}_{2} \mathrm{O}_{3}(30.5 \mathrm{mg}, 0.3$ mmol), the mixture was reflux at $65{ }^{\circ} \mathrm{C}$ for $14 \mathrm{~h}$, after the reaction was accomplished, cooled to temperature and filtration to remove $\mathrm{Al}_{2} \mathrm{O}_{3}$, washed with $\mathrm{EA}$, the combined organic layer was concentrated under reduced pressure to give the crude product. The crude product washed with $\mathrm{H}_{2} \mathrm{O}(1 \times 5$ $\mathrm{mL})$, extracted with EA $(1 \times 5 \mathrm{~mL})$, organic layers were dried over $\mathrm{Na}_{2} \mathrm{SO}_{4}$, filtered and concentrated under reduced pressure and purified by flash chromatography $(\mathrm{PE} / \mathrm{EtOAc}=5: 1)$ to afford product $\mathbf{8}(22.7$ $\mathrm{mg}, 41 \%)^{[6]} .{ }^{1} \mathrm{H}$ NMR $(400 \mathrm{MHz}$, Chloroform- $d) \delta 7.77(\mathrm{~d}, J=8.4 \mathrm{~Hz}, 2 \mathrm{H}), 7.31(\mathrm{~d}, J=8.0 \mathrm{~Hz}, 2 \mathrm{H}), 7.28$ -7.20 (m, 5H), 5.18 (dd, $J=10.0,1.2 \mathrm{~Hz}, 1 \mathrm{H}), 3.42(\mathrm{~d}, J=10.0 \mathrm{~Hz}, 1 \mathrm{H}), 3.39$ (d, $J=10.4 \mathrm{~Hz}, 1 \mathrm{H}), 3.25$ (dd, $J=14.0,1.6 \mathrm{~Hz}, 1 \mathrm{H}), 2.40$ (s, 3H). ${ }^{13} \mathrm{C}$ NMR $(101 \mathrm{MHz}$, Chloroform- $d$ ) $\delta$ 145.3, 140.7, 136.2, 130.1, 128.8, 128.3, 128.0, 125.7, 68.5, 64.0, 21.7. HRMS (ESI/Q-TOF) m/z: $[\mathrm{M}+\mathrm{Na}]^{+}$calcd for $\mathrm{C}_{15} \mathrm{H}_{16} \mathrm{NaO}_{3} \mathrm{~S}$ 299.0715; found: 299.0715 . 


\section{Reference}

[1] Li, Y.; Jiang, X.; Zhao, C.; Fu, X.; Xu, X.; Tang, P. ACS Catal. 2017, 7, 1606-1609.

[2] Hossain, A.; Engl, S.; Lutsker, E.; Reiser, O. ACS Catal. 2019, 9, 1103-1109.

[3] Niu, T.; Lin, D.; Xue, L.; Jiang, D.; Ni, B. Synlett 2017, 28, 364-368.

[4] Alkan-Zambada, M.; Hu, X.; J. Org. Chem. 2019, 84, 4525-4533.

[5] Percec, V.; Barboiu. B.; Kim, H. J. J. Am. Chem. Soc. 1998, 120, 305-316.

[6] Li, C.; Xia, F.; Zhang, W.; Wang, K.; Xu, G.; Qin H. Tetrahedron Letters 2018, 59, 2607-2609. 
Copies of ${ }^{1} \mathrm{H}$ NMR, ${ }^{19}$ F NMR and ${ }^{13} \mathrm{C}$ NMR spectra of Products

(S)-1-((2-chloro-2-phenylethyl)sulfonyl)-4-methylbenzene
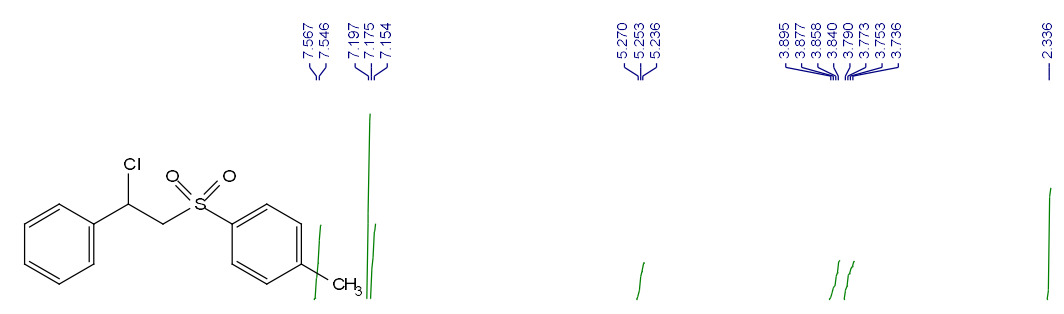

$4 a$

${ }^{1} \mathrm{H}$ NMR $\left(400 \mathrm{MHz}, \mathrm{CDCl}_{3}\right)$
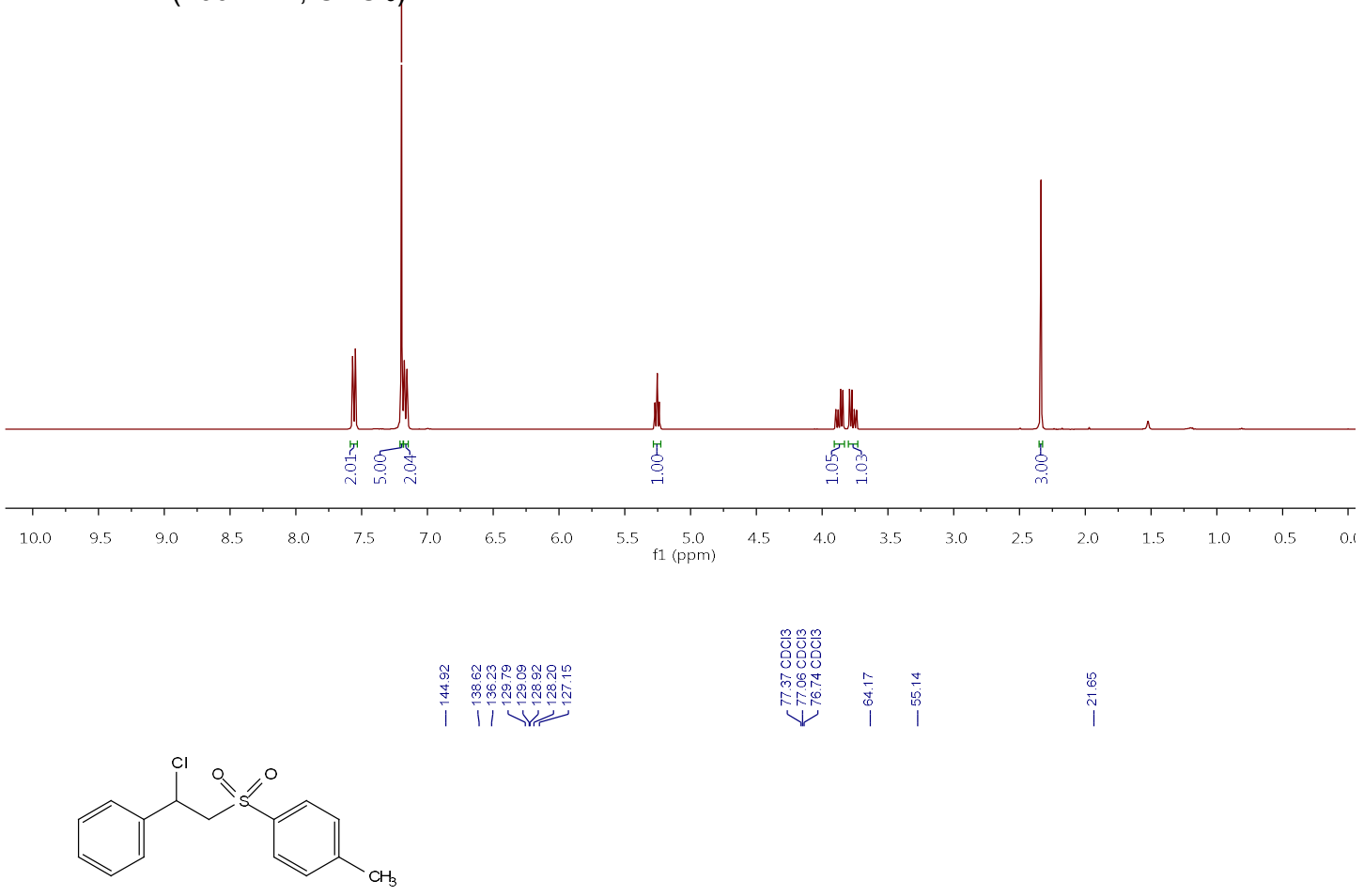

$4 a$

${ }^{13} \mathrm{C}$ NMR $\left(101 \mathrm{MHz}, \mathrm{CDCl}_{3}\right)$

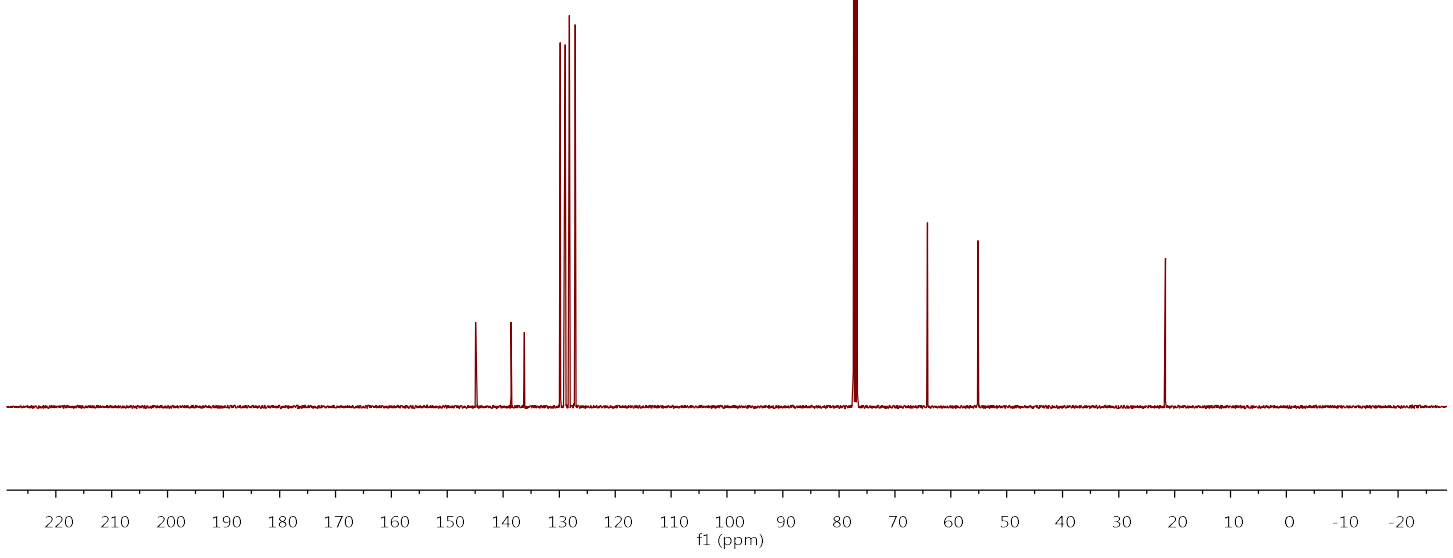

$\mathrm{S} 15$ 
(R)-4-((2-chloro-2-phenylethyl)sulfonyl)-1,1'-biphenyl

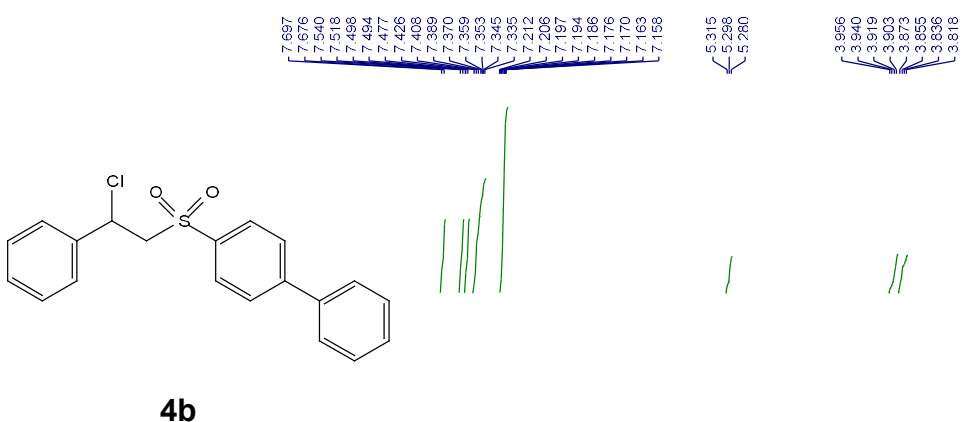

${ }^{1} \mathrm{H}$ NMR $\left(400 \mathrm{MHz}, \mathrm{CDCl}_{3}\right)$
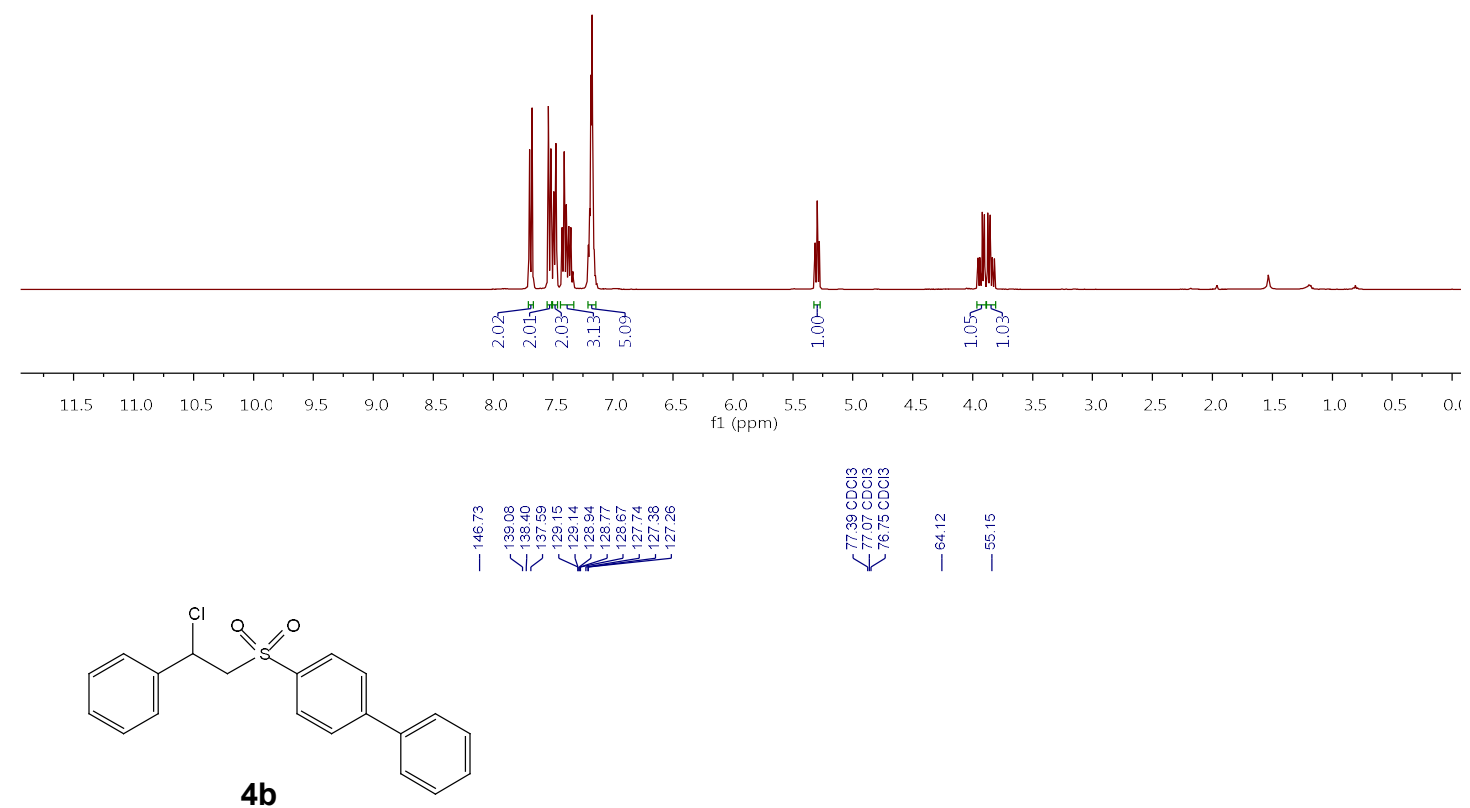

${ }^{13} \mathrm{C}$ NMR $\left(101 \mathrm{MHz}, \mathrm{CDCl}_{3}\right)$

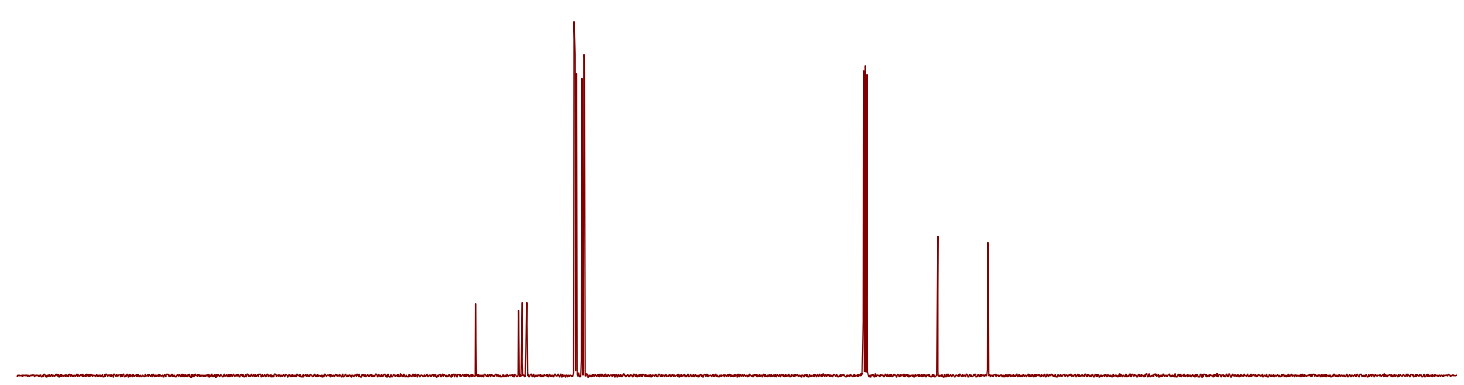

$\begin{array}{llllllllllllllllllllllllll}220 & 210 & 200 & 190 & 180 & 170 & 160 & 150 & 140 & 130 & 120 & 110 & \underset{f 1}{100}(\mathrm{ppm}) & 90 & 80 & 70 & 60 & 50 & 40 & 30 & 20 & 10 & 0 & -10 & -20\end{array}$ 
(S)-1-((2-chloro-2-phenylethyl)sulfonyl)naphthalene

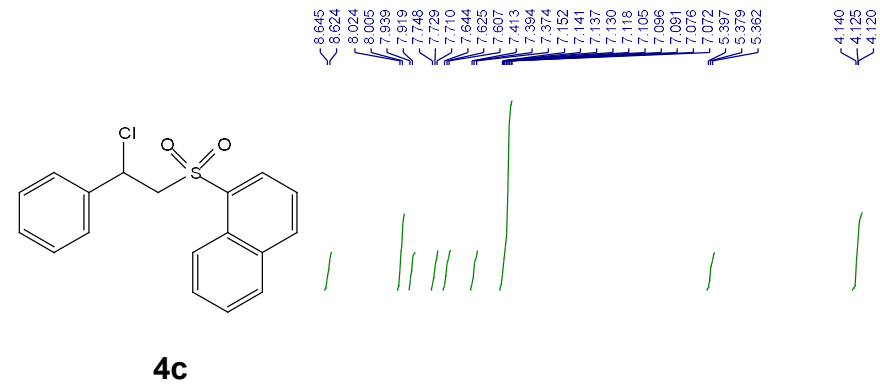

${ }^{1} \mathrm{H} \mathrm{NMR}\left(400 \mathrm{MHz}, \mathrm{CDCl}_{3}\right)$
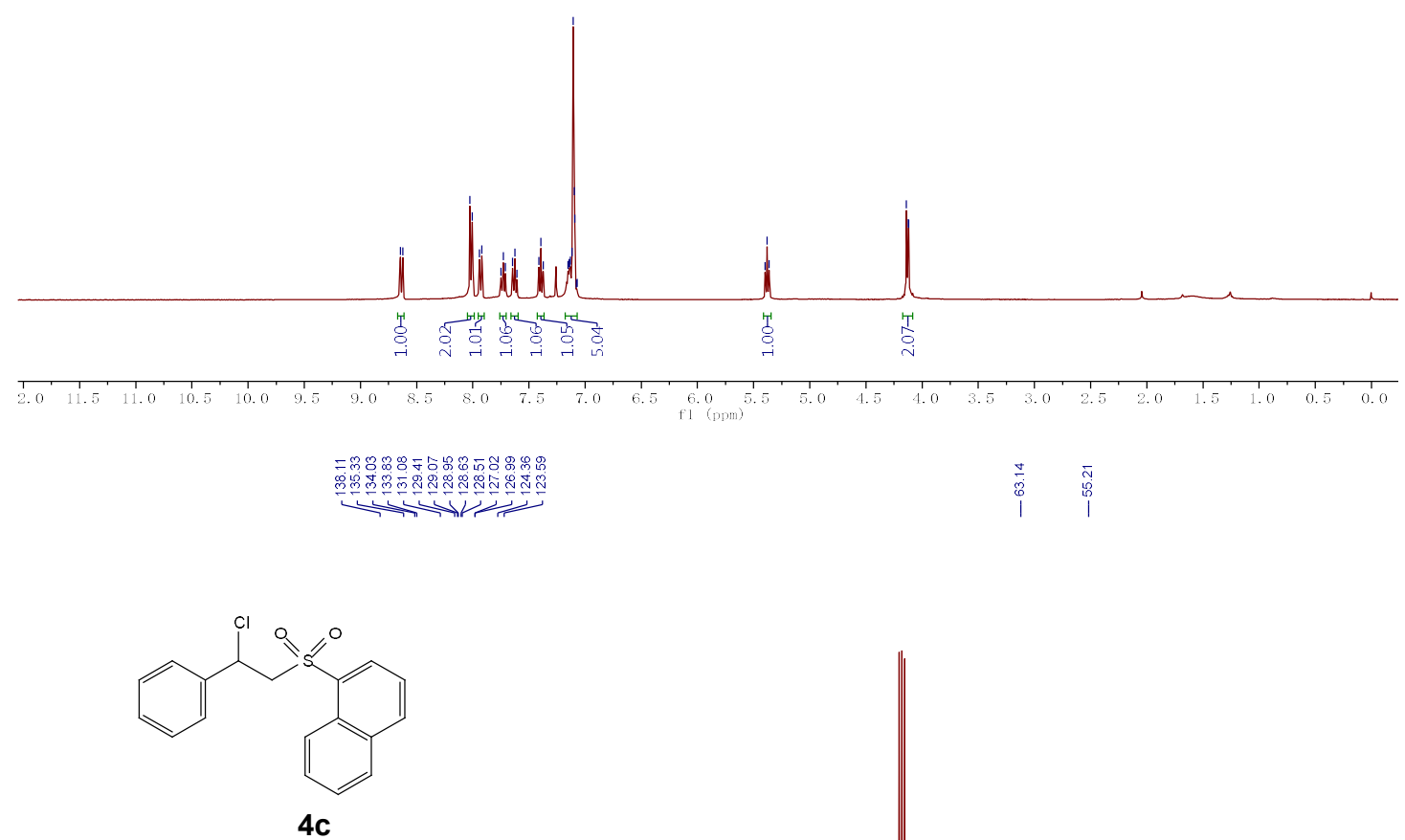

${ }^{13} \mathrm{C}$ NMR $\left(101 \mathrm{MHz}, \mathrm{CDCl}_{3}\right)$

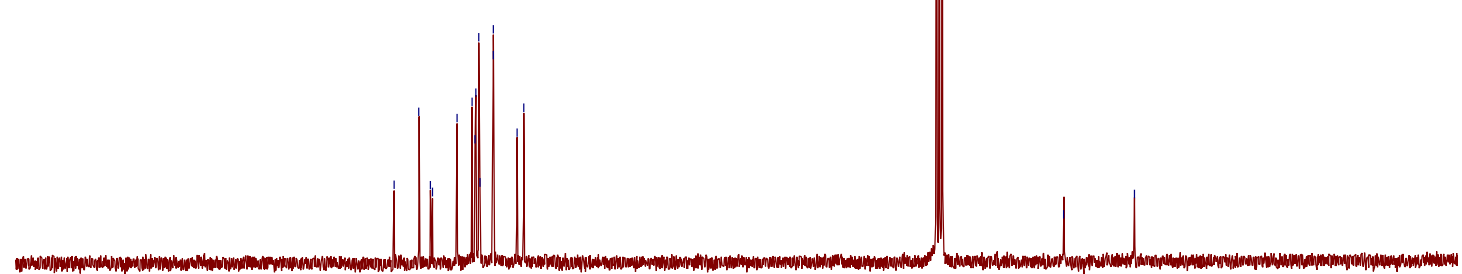


(S)-2-((2-([1,1'-biphenyl]-4-yl)-2-chloroethyl)sulfonyl)naphthalene

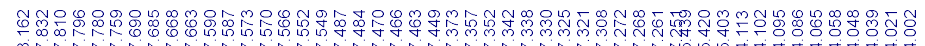

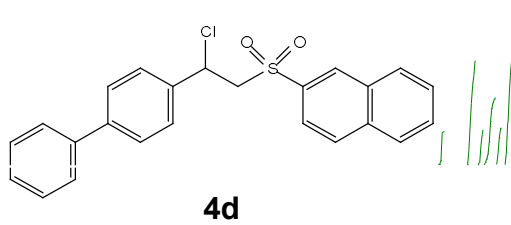

${ }^{1} \mathrm{H}$ NMR $\left(400 \mathrm{MHz}, \mathrm{CDCl}_{3}\right)$

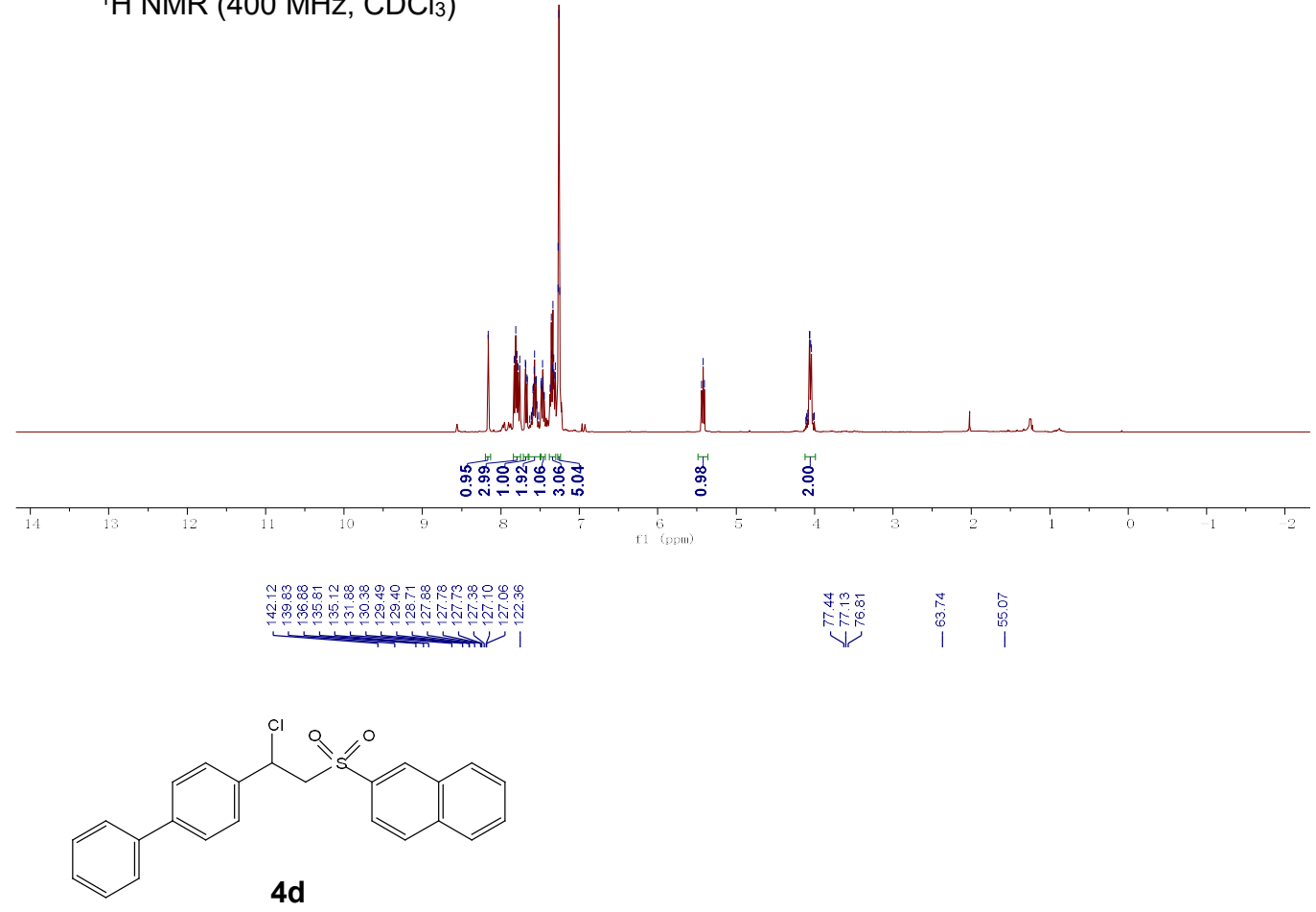

${ }^{13} \mathrm{C}$ NMR $\left(101 \mathrm{MHz}, \mathrm{CDCl}_{3}\right)$

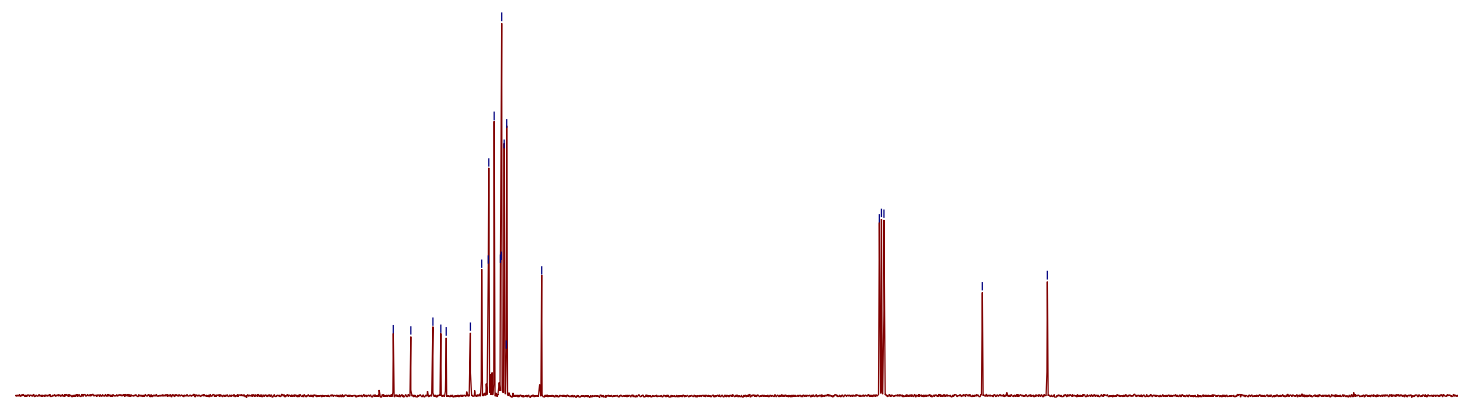

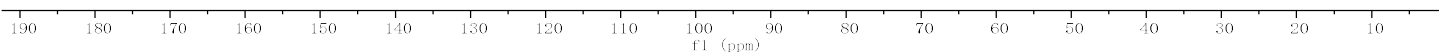


(S)-4-((2-chloro-2-phenylethyl)sulfonyl)-1,2-dimethylbenzene

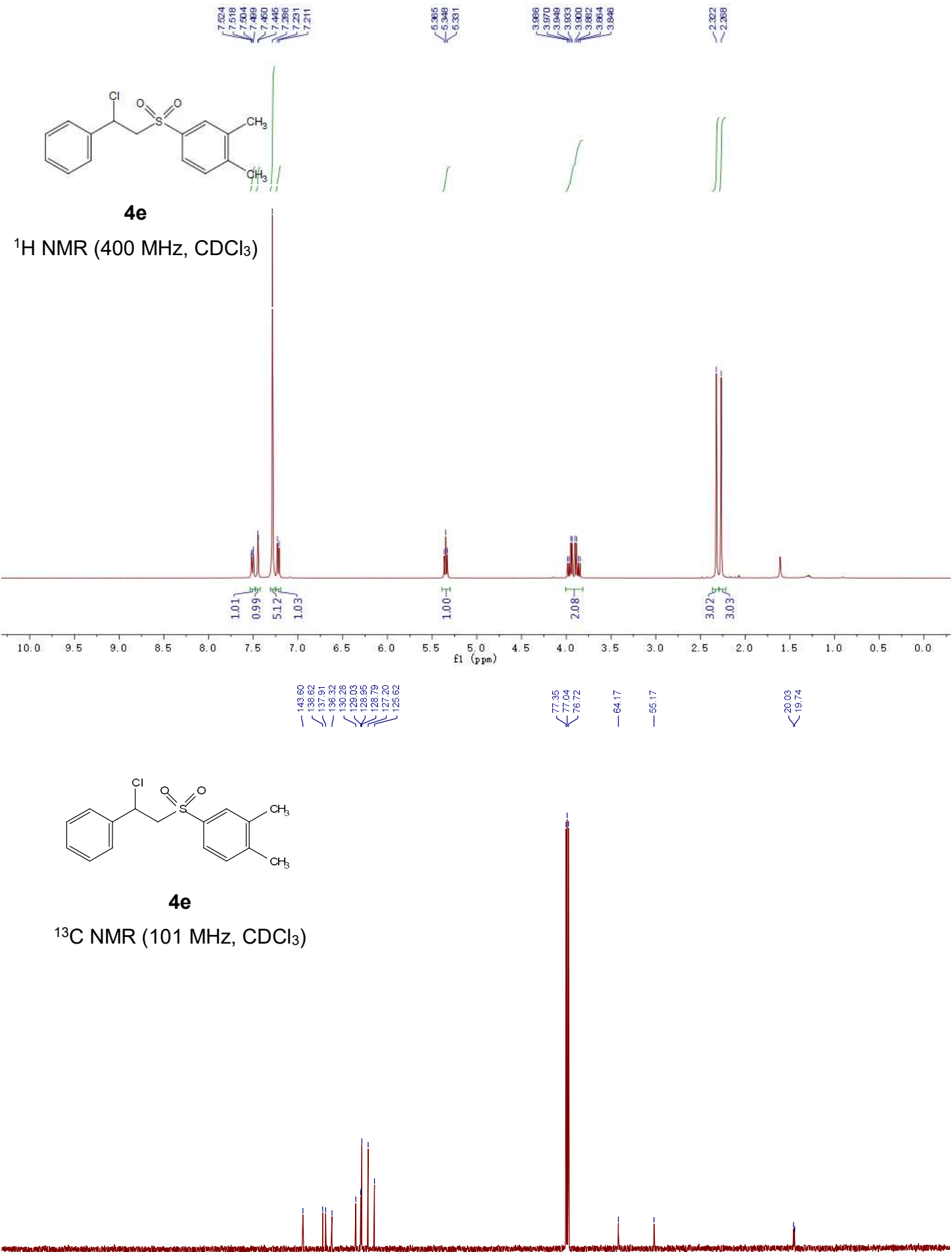


1-(tert-butyl)-4-((2-chloro-2-phenylethyl)sulfonyl)benzene

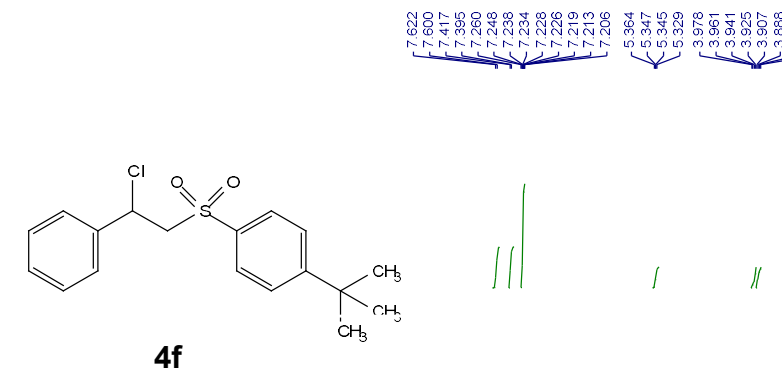

${ }^{1} \mathrm{H}$ NMR $\left(400 \mathrm{MHz}, \mathrm{CDCl}_{3}\right)$

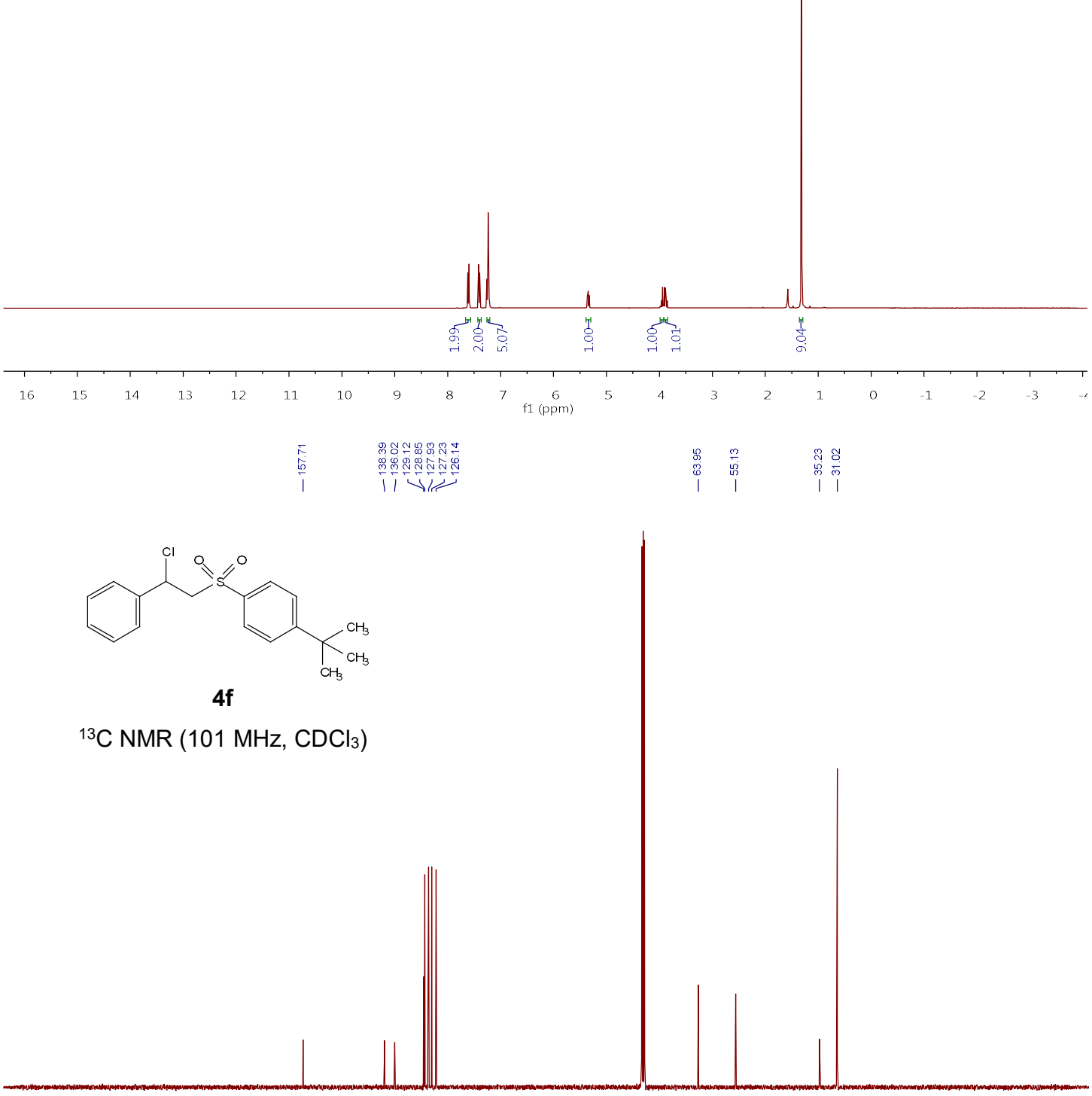

$\begin{array}{lllllllllllllllllllllllll}220 & 210 & 200 & 190 & 18 \mathrm{C} & 170 & 16 \mathrm{C} & 150 & 14 \mathrm{C} & 130 & 120 & 110 & \begin{array}{c}100 \\ \mathrm{f} 1(\mathrm{ppm})\end{array} & 90 & 80 & 70 & 60 & 50 & 40 & 30 & 20 & 10 & 0 & -1 \mathrm{C} & -20\end{array}$ 
(R)-1-((2-chloro-2-phenylethyl)sulfonyl)-4-methoxybenzene

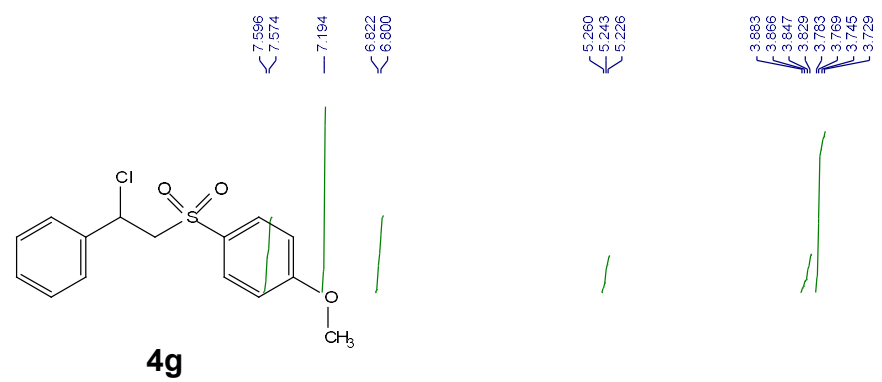

${ }^{1} \mathrm{H}$ NMR $\left(400 \mathrm{MHz}, \mathrm{CDCl}_{3}\right)$

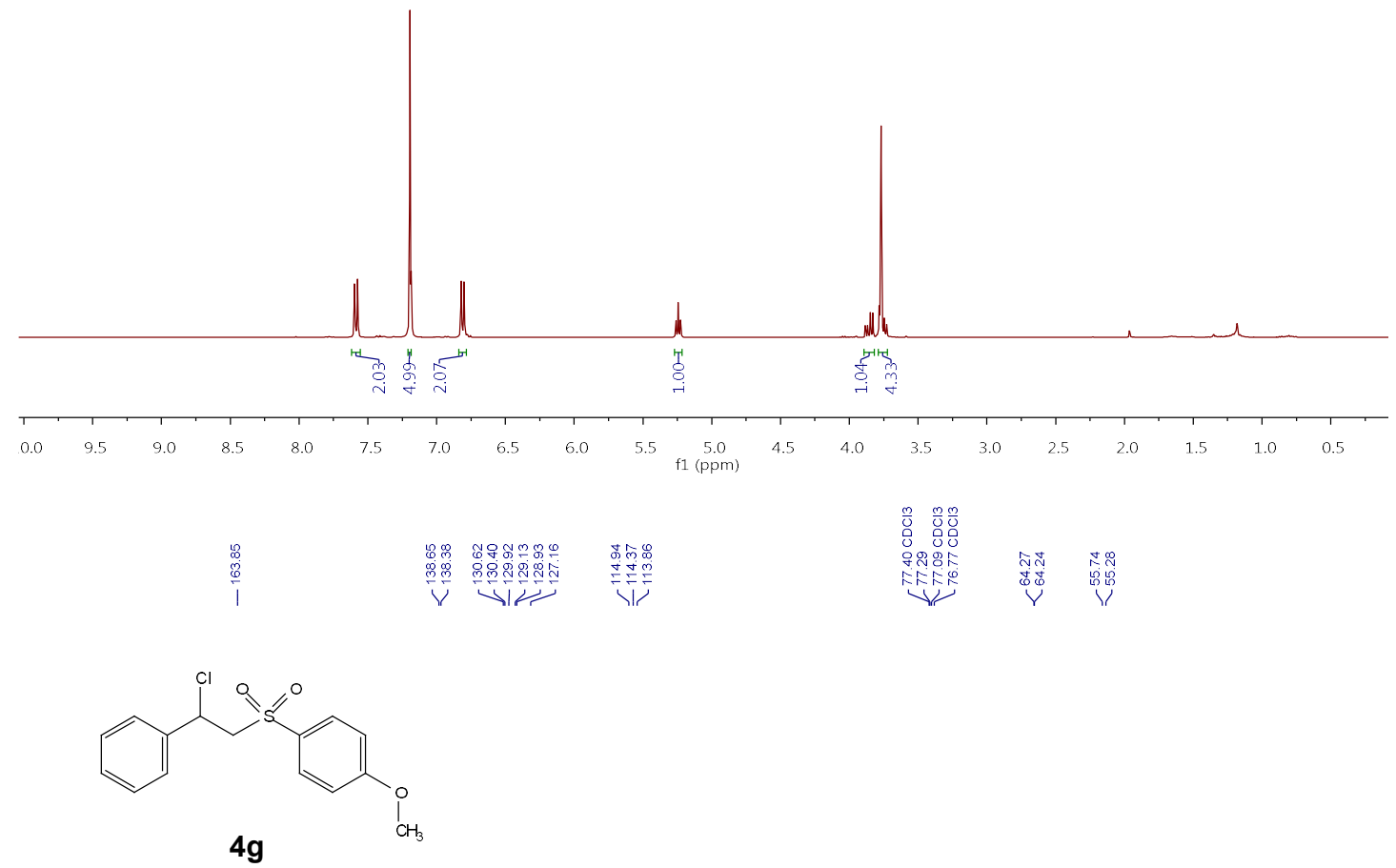

${ }^{13} \mathrm{C}$ NMR $\left(101 \mathrm{MHz}, \mathrm{CDCl}_{3}\right)$
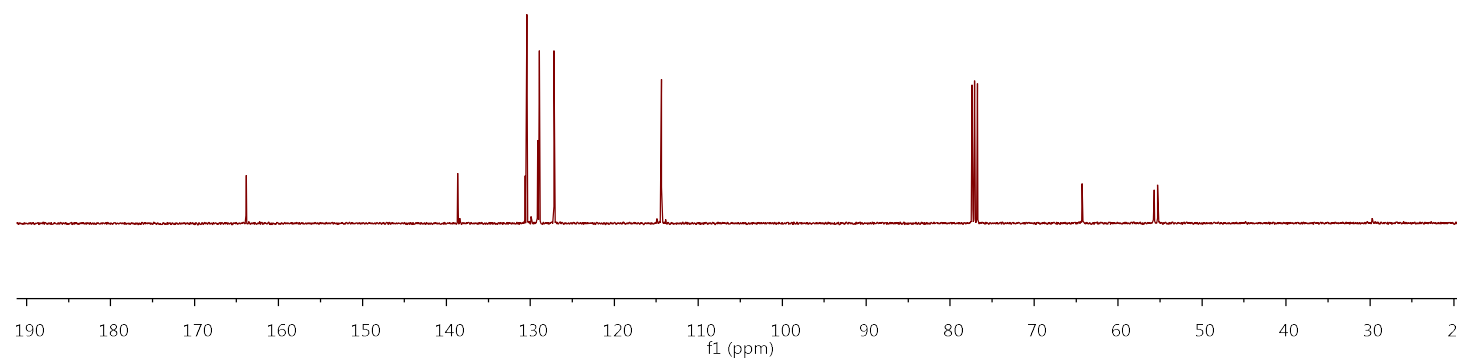
(S)-2-(1-chloro-2-((4-(trifluoromethoxy)phenyl)sulfonyl)ethyl)naphthalene
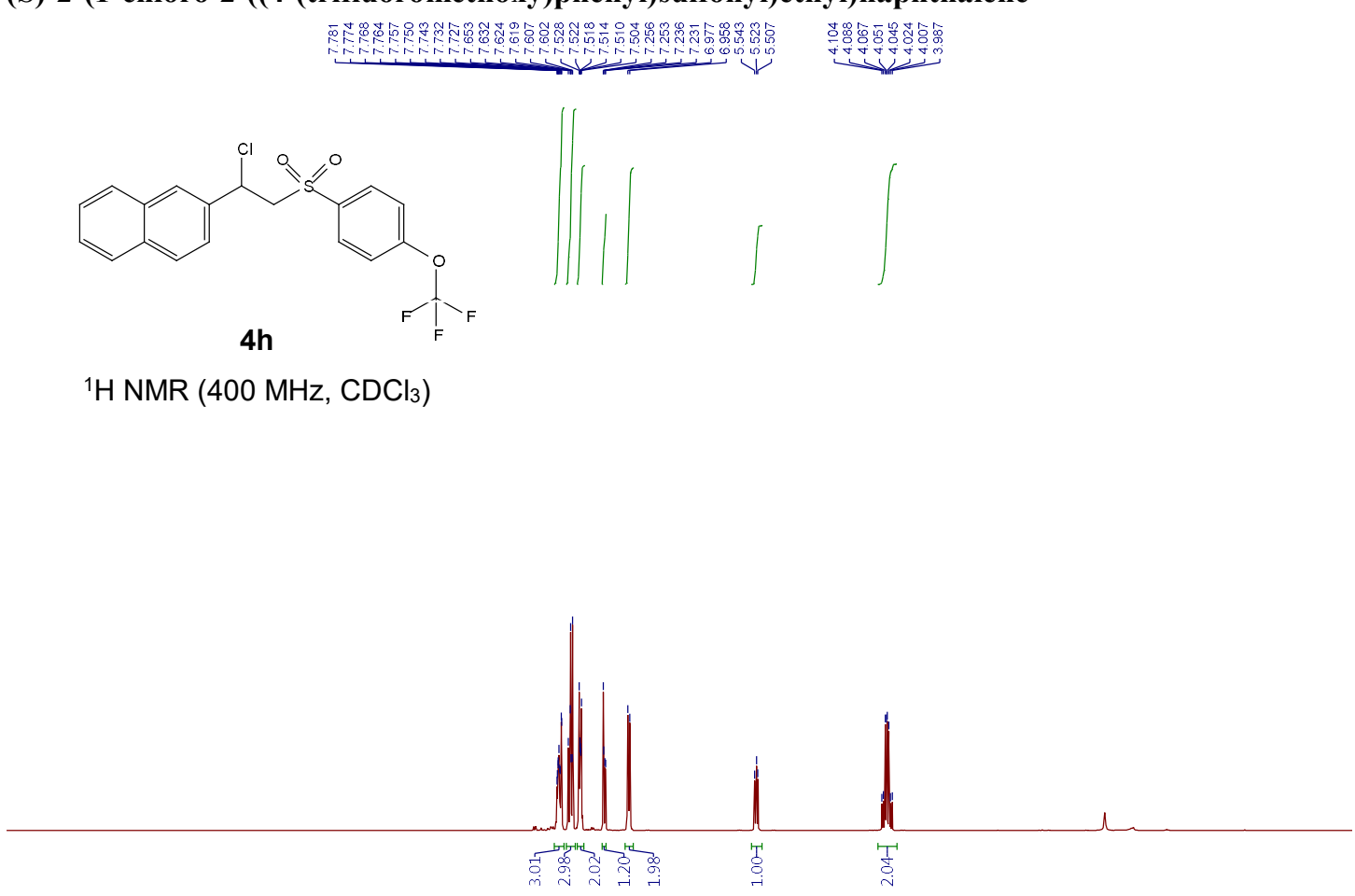

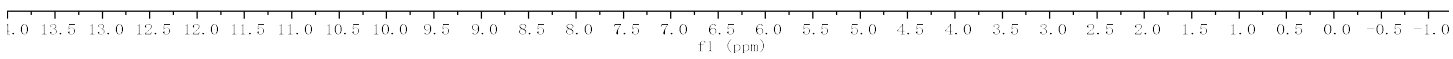

$\stackrel{\infty}{2}$
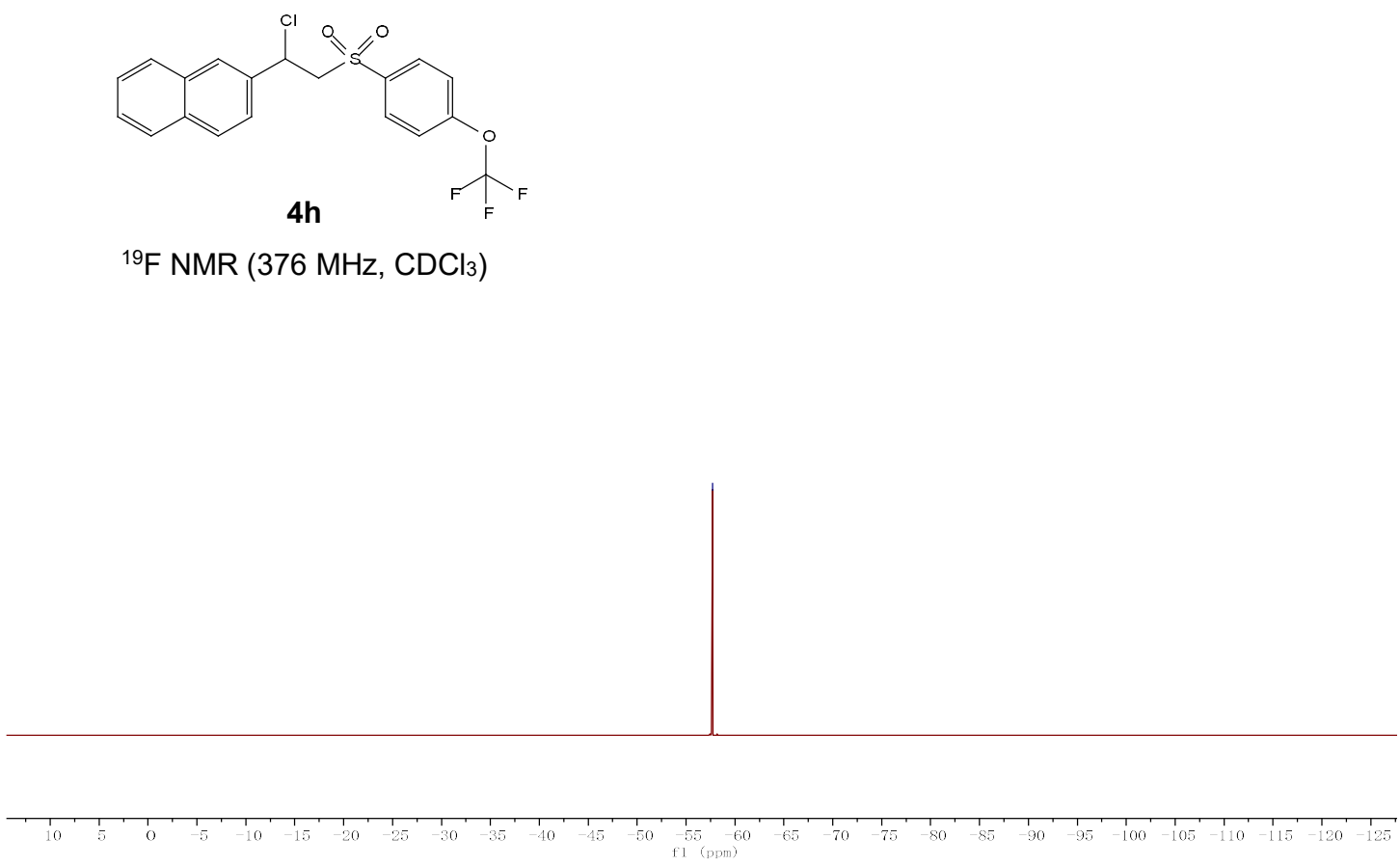

S22 


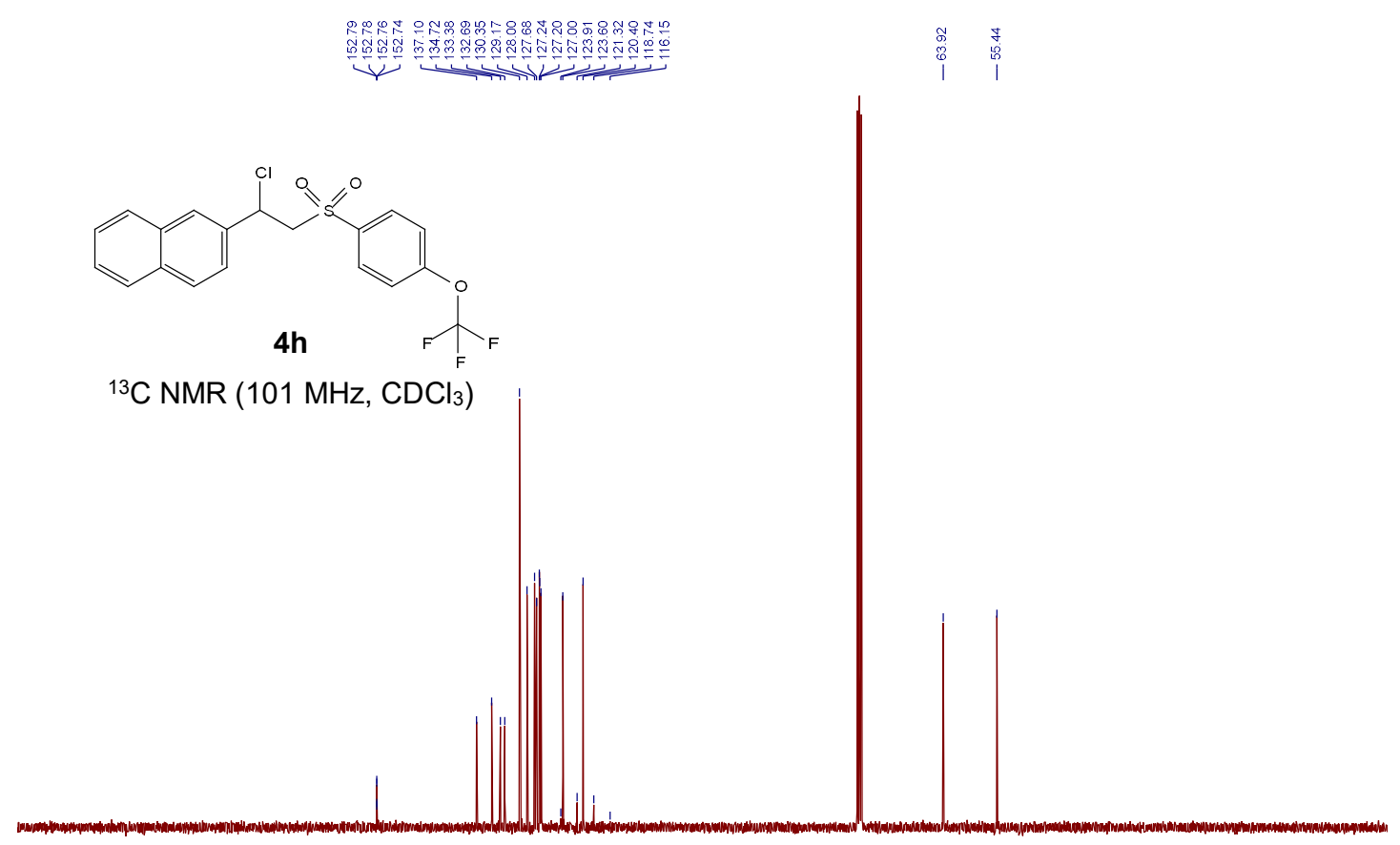


(R)-1-((2-chloro-2-phenylethyl)sulfonyl)-4-phenoxybenzene

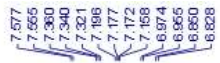

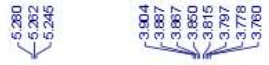

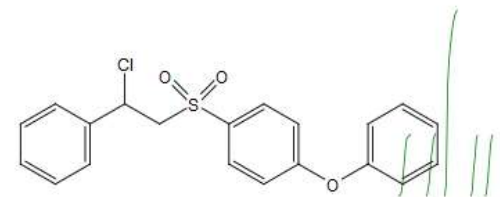

$4 \mathbf{i}$

${ }^{1} \mathrm{H}$ NMR $\left(400 \mathrm{MHz}, \mathrm{CDCl}_{3}\right)$

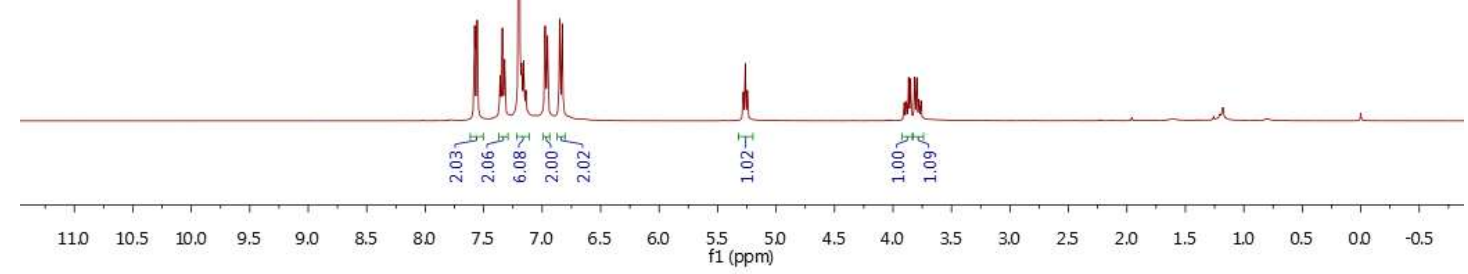

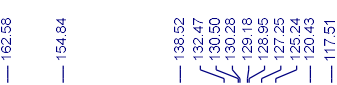
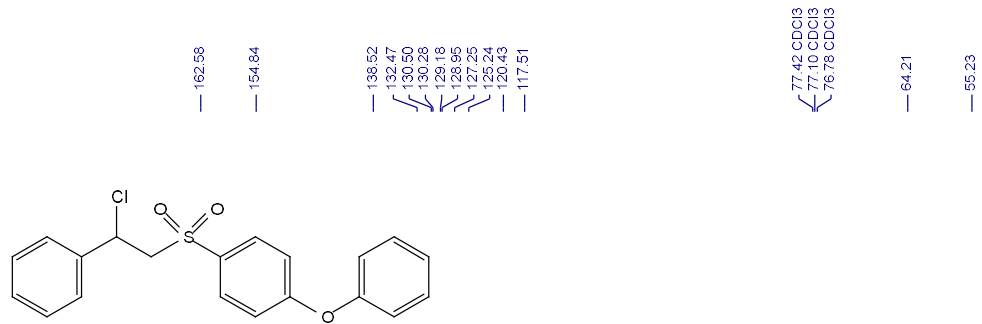

$4 i$

${ }^{13} \mathrm{C}$ NMR $\left(101 \mathrm{MHz}, \mathrm{CDCl}_{3}\right)$

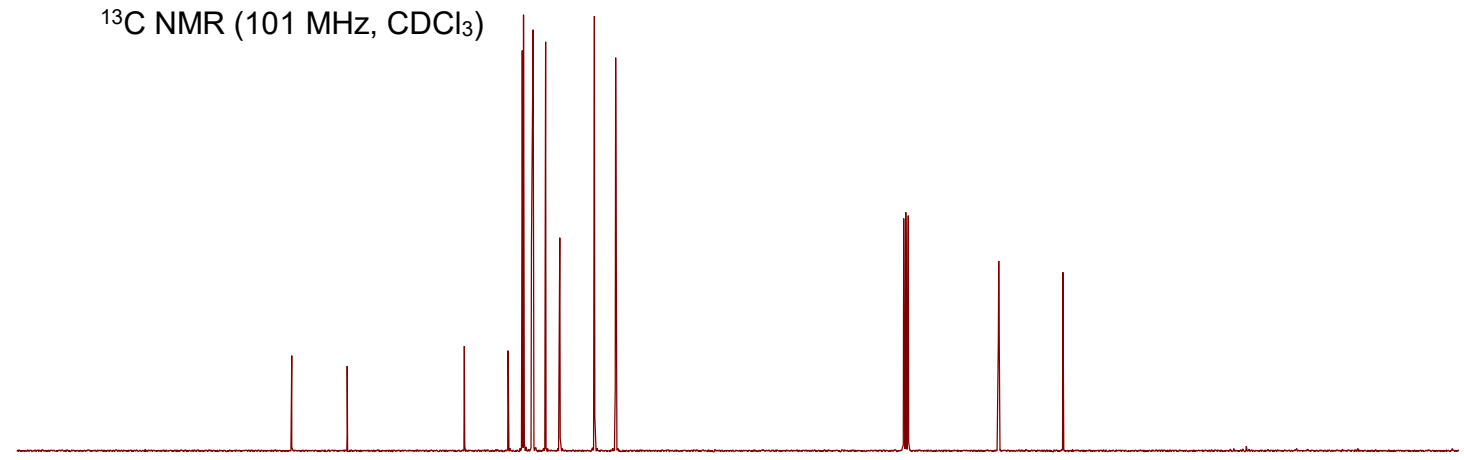

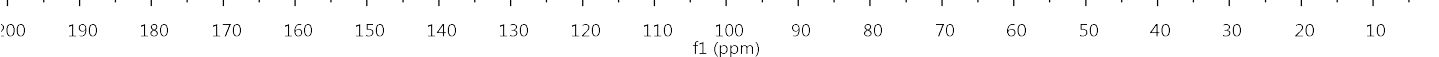



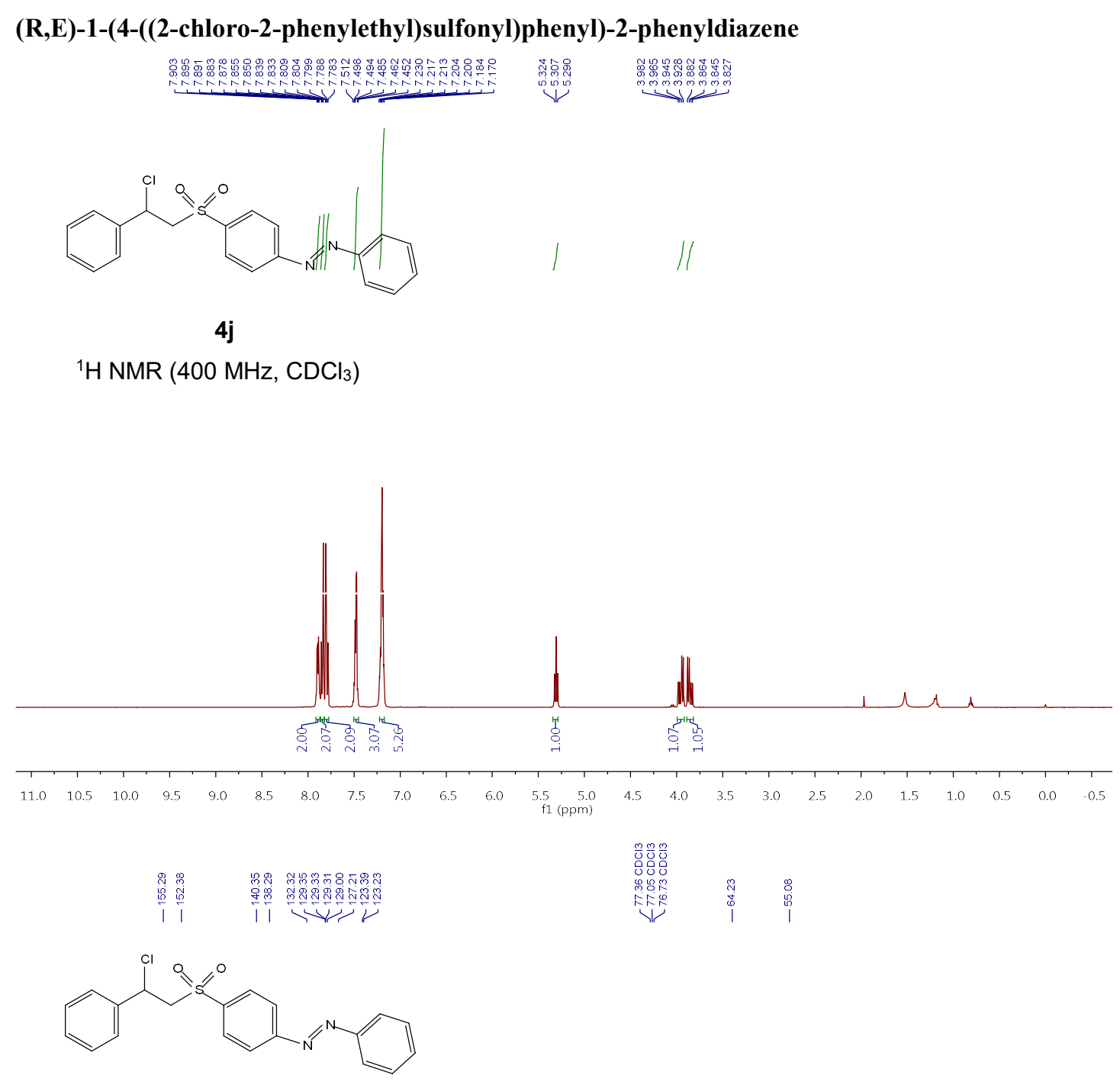

4j

${ }^{13} \mathrm{C}$ NMR (101 MHz, $\mathrm{CDCl}_{3}$ )

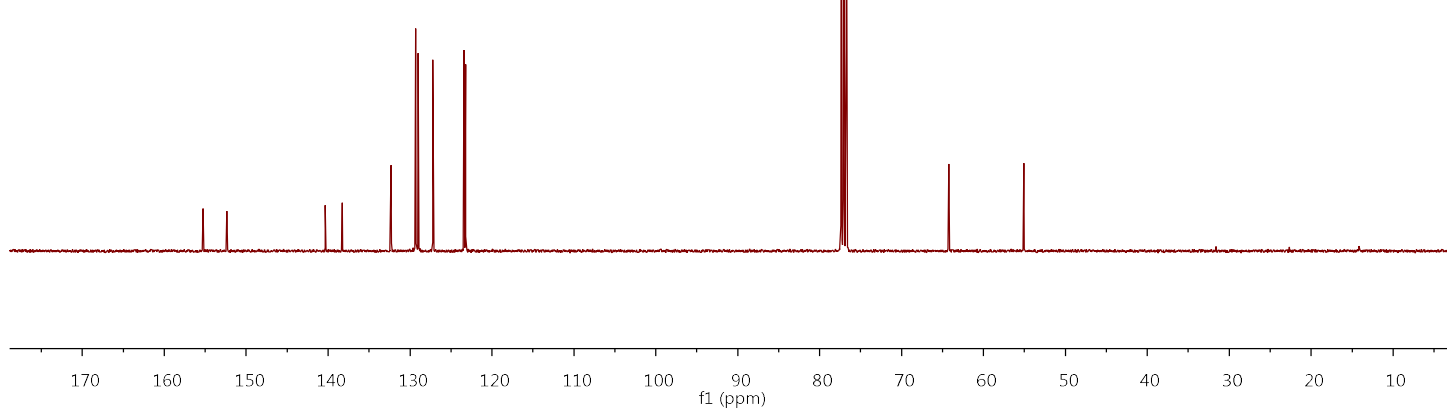

S25 
(R)-1-((2-chloro-2-phenylethyl)sulfonyl)-4-fluorobenzene

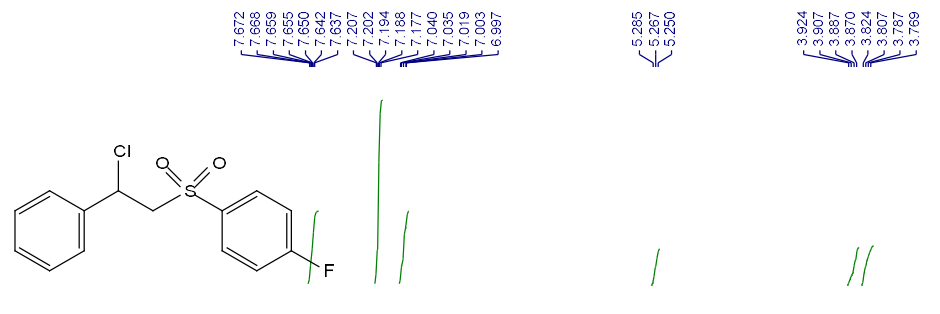

4k

${ }^{1} \mathrm{H}$ NMR $\left(400 \mathrm{MHz}, \mathrm{CDCl}_{3}\right)$
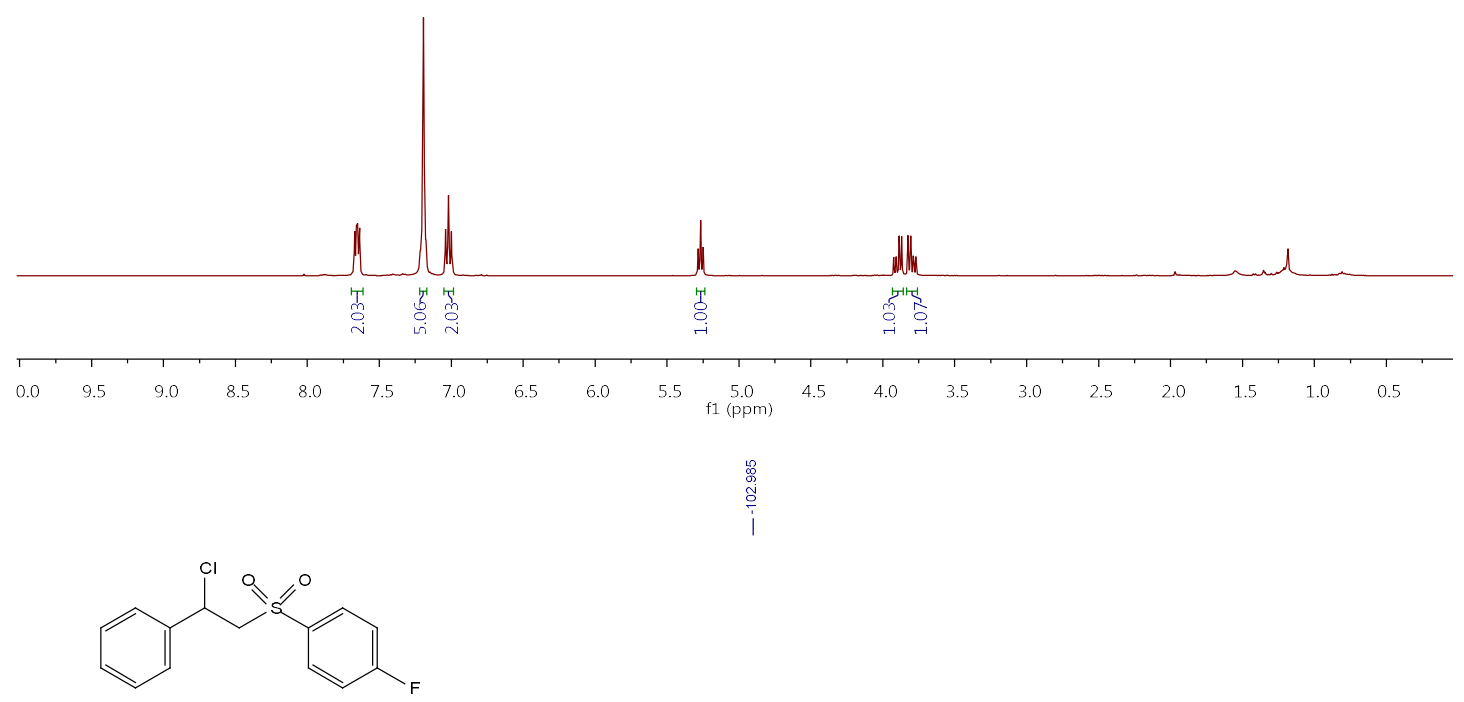

4k

${ }^{19} \mathrm{~F} \mathrm{NMR}\left(376 \mathrm{MHz}, \mathrm{CDCl}_{3}\right)$

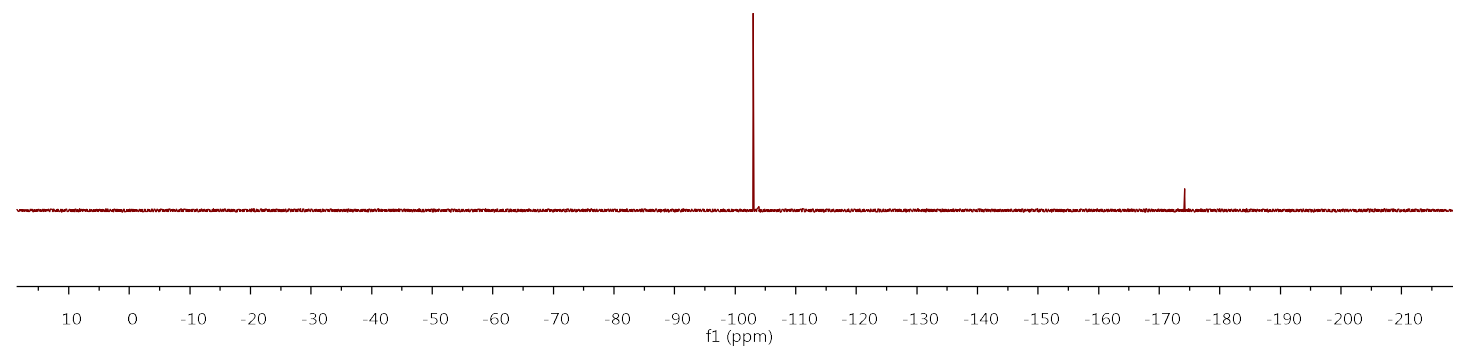



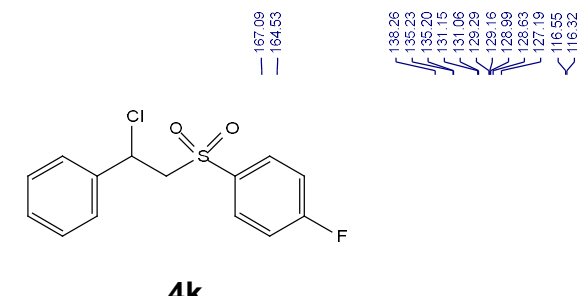

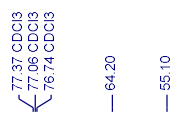

${ }^{13} \mathrm{C}$ NMR (101 MHz, $\left.\mathrm{CDCl}_{3}\right)$

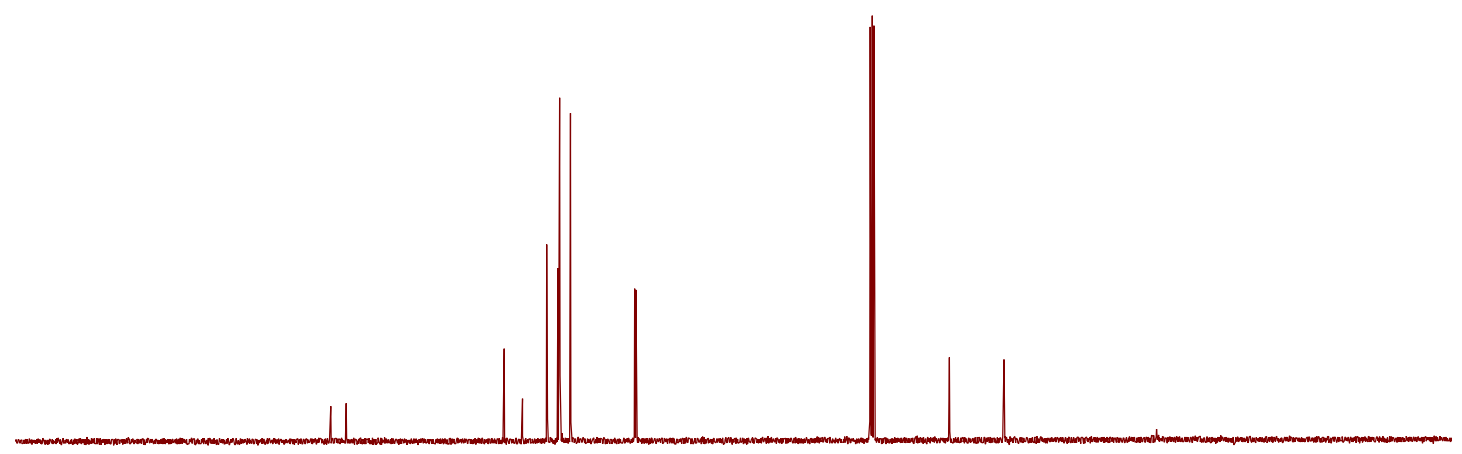

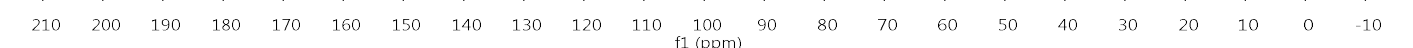


(S)-1-chloro-4-((2-chloro-2-phenylethyl)sulfonyl)benzene

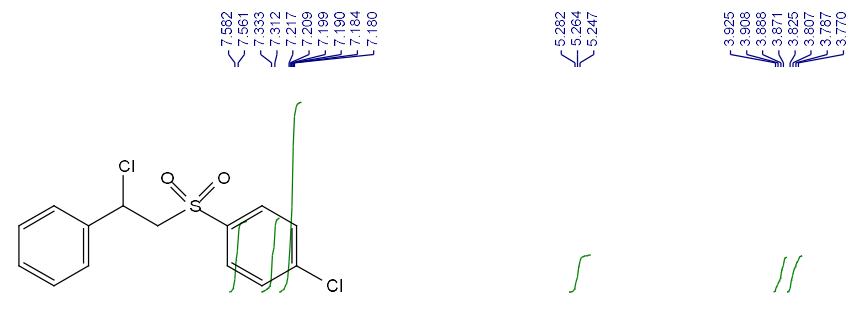

4

${ }^{1} \mathrm{H}$ NMR $\left(400 \mathrm{MHz}, \mathrm{CDCl}_{3}\right)$
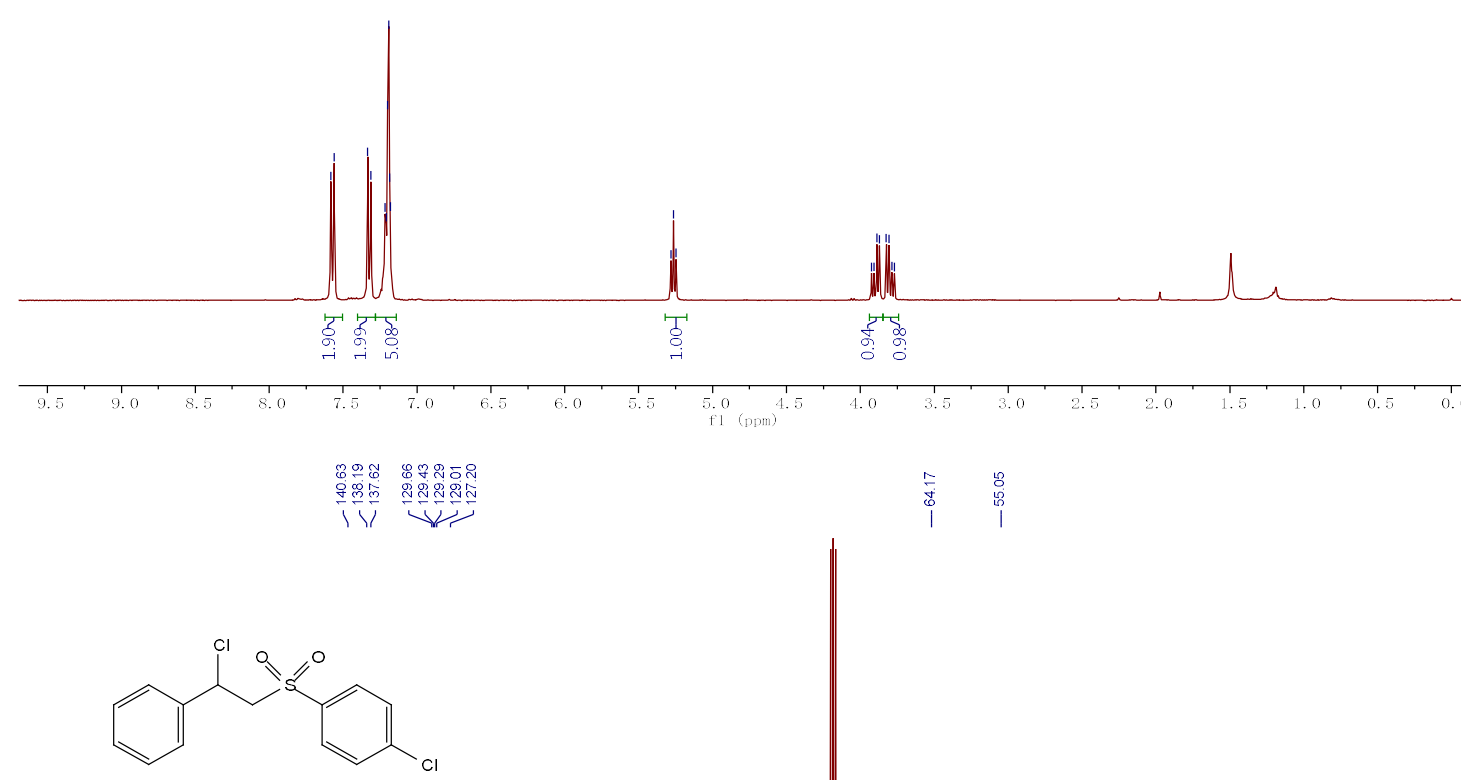

4!

$\left.{ }^{13} \mathrm{C} \mathrm{NMR} \mathrm{(101} \mathrm{MHz,} \mathrm{CDCl}_{3}\right)$

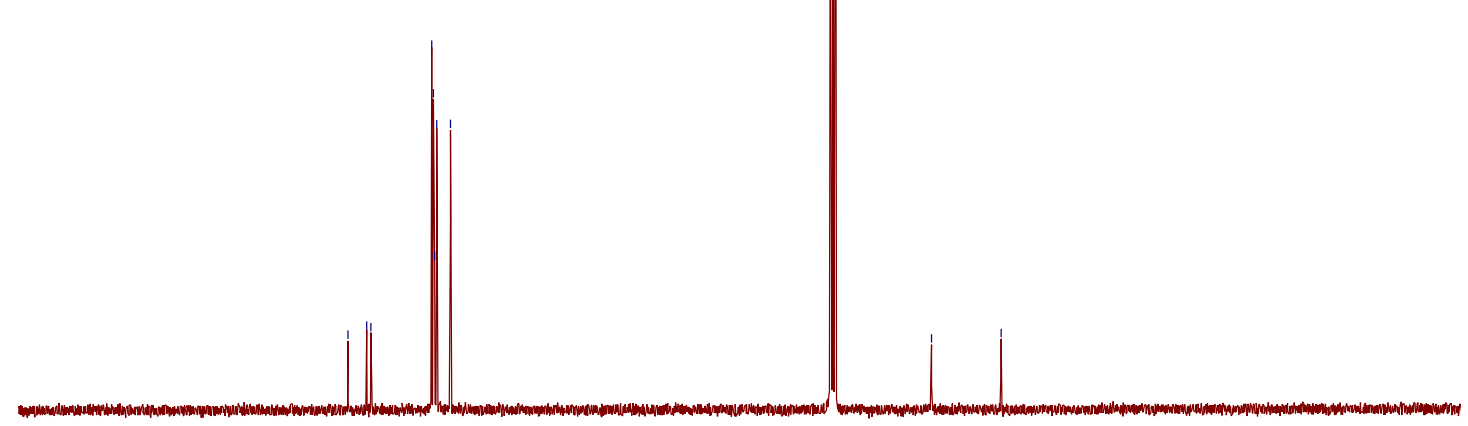


(S)-1-((2-chloro-2-phenylethyl)sulfonyl)-3-iodobenzene

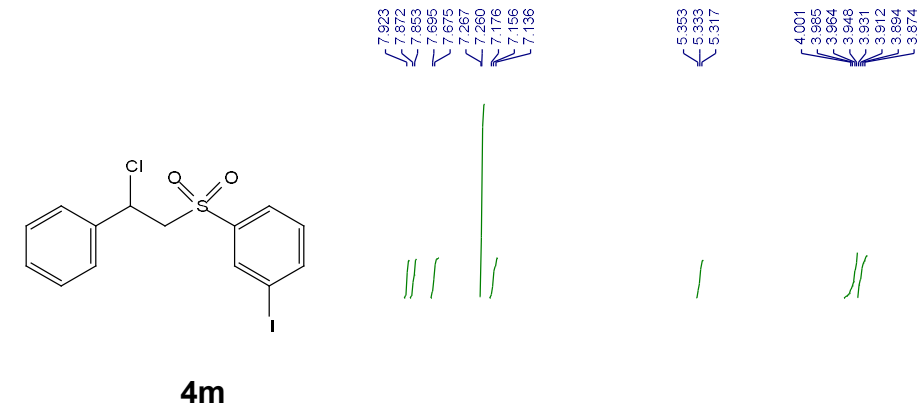

${ }^{1} \mathrm{H}$ NMR $\left(400 \mathrm{MHz}, \mathrm{CDCl}_{3}\right)$

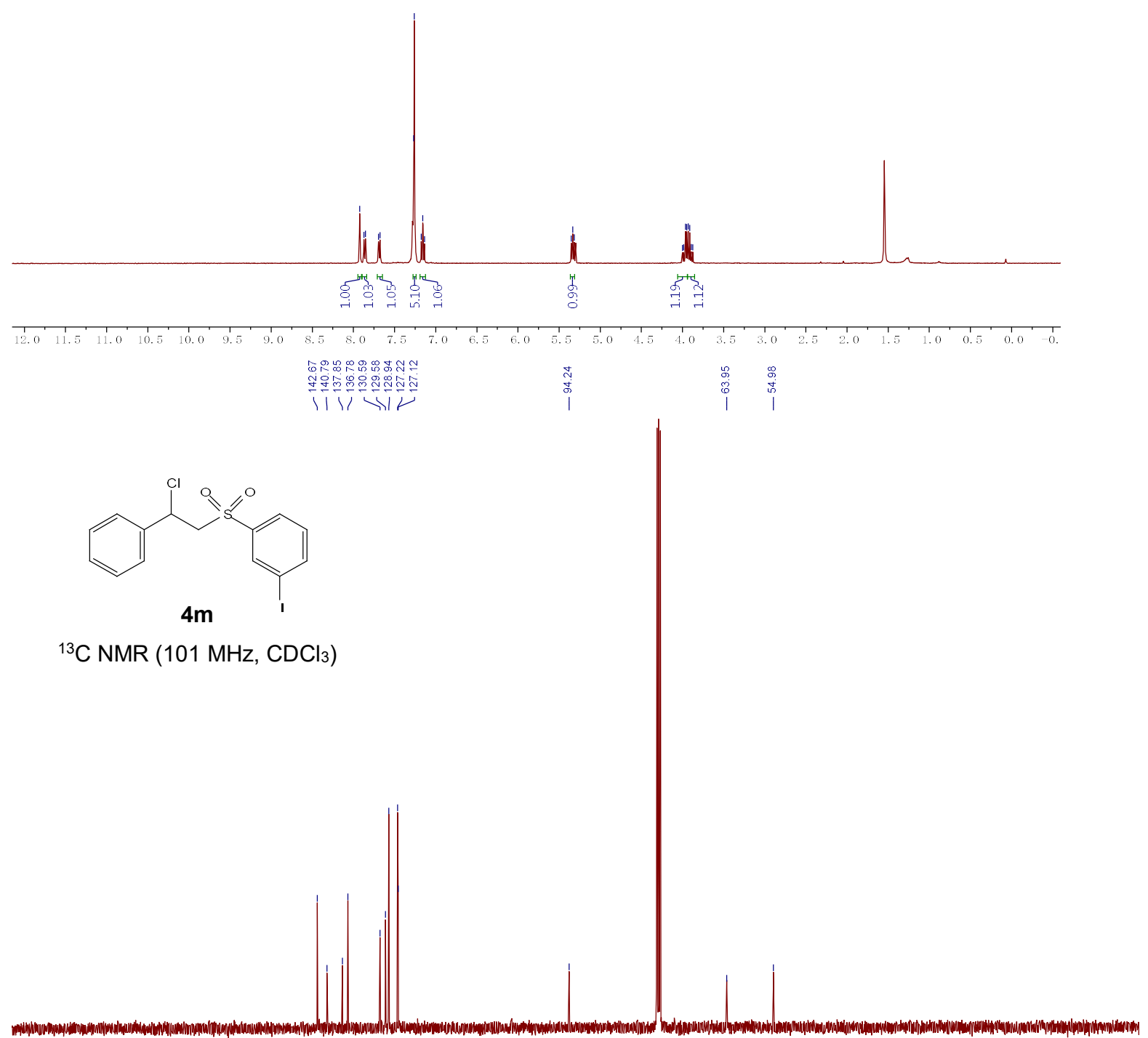

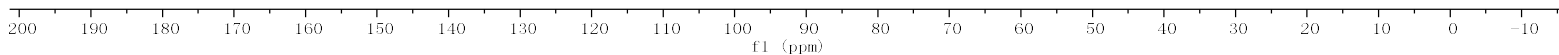


(S)-1-((2-chloro-2-phenylethyl)sulfonyl)-4-(methylsulfonyl)benzene

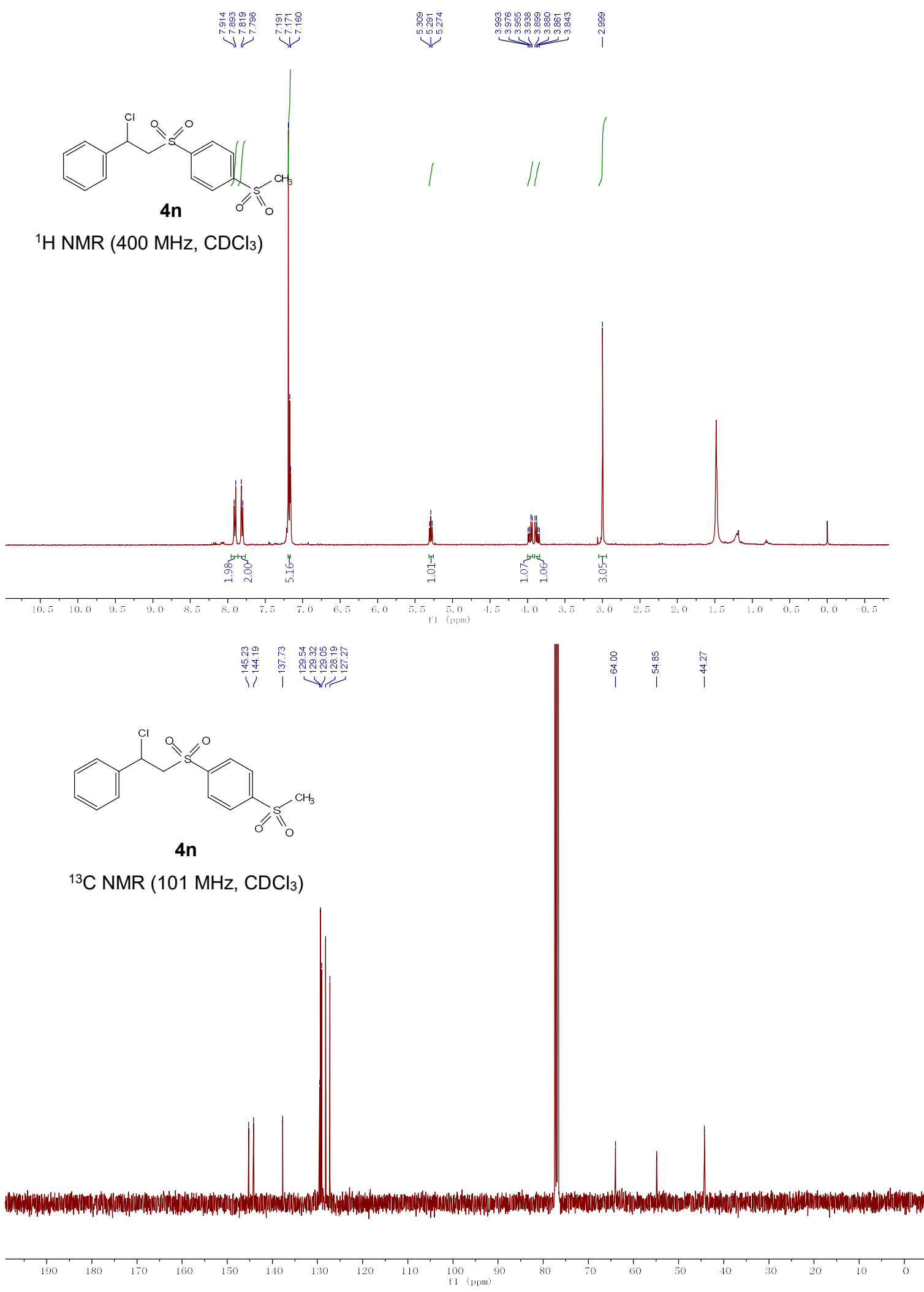

$\mathrm{S} 30$ 
(S)-1-((2-chloro-2-phenylethyl)sulfonyl)-4-(trifluoromethyl)benzene
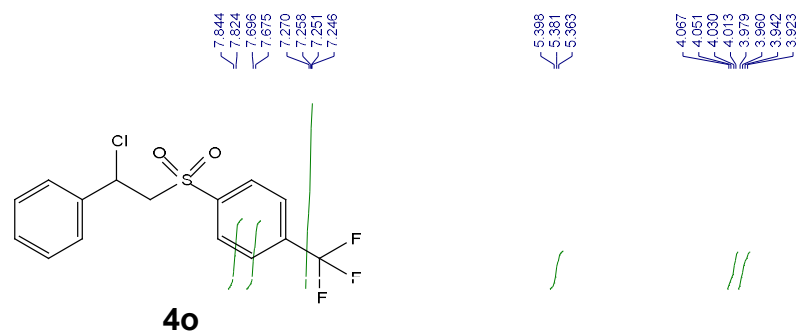

${ }^{1} \mathrm{H}$ NMR $\left(400 \mathrm{MHz}, \mathrm{CDCl}_{3}\right)$

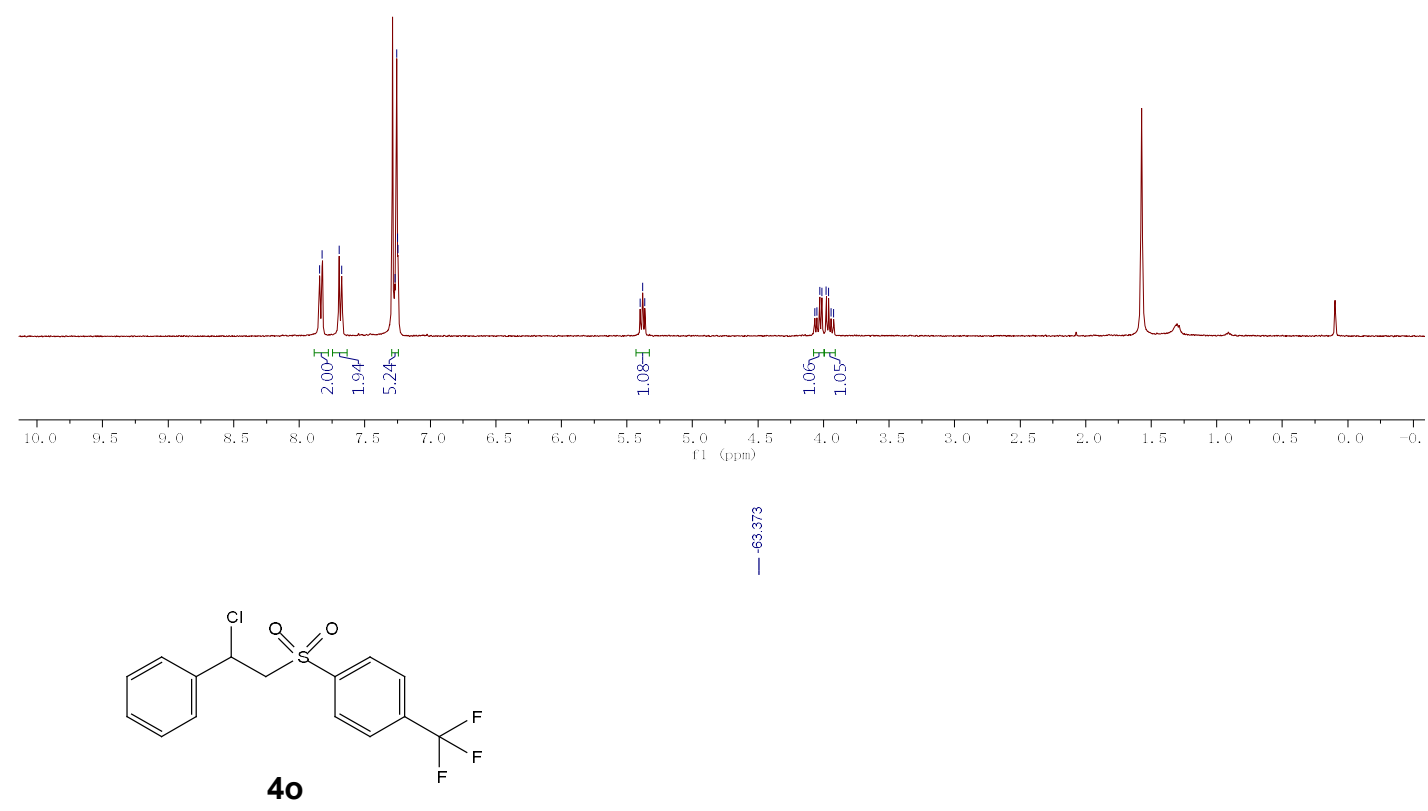

${ }^{19} \mathrm{~F}$ NMR (376 $\left.\mathrm{MHz}, \mathrm{CDCl}_{3}\right)$

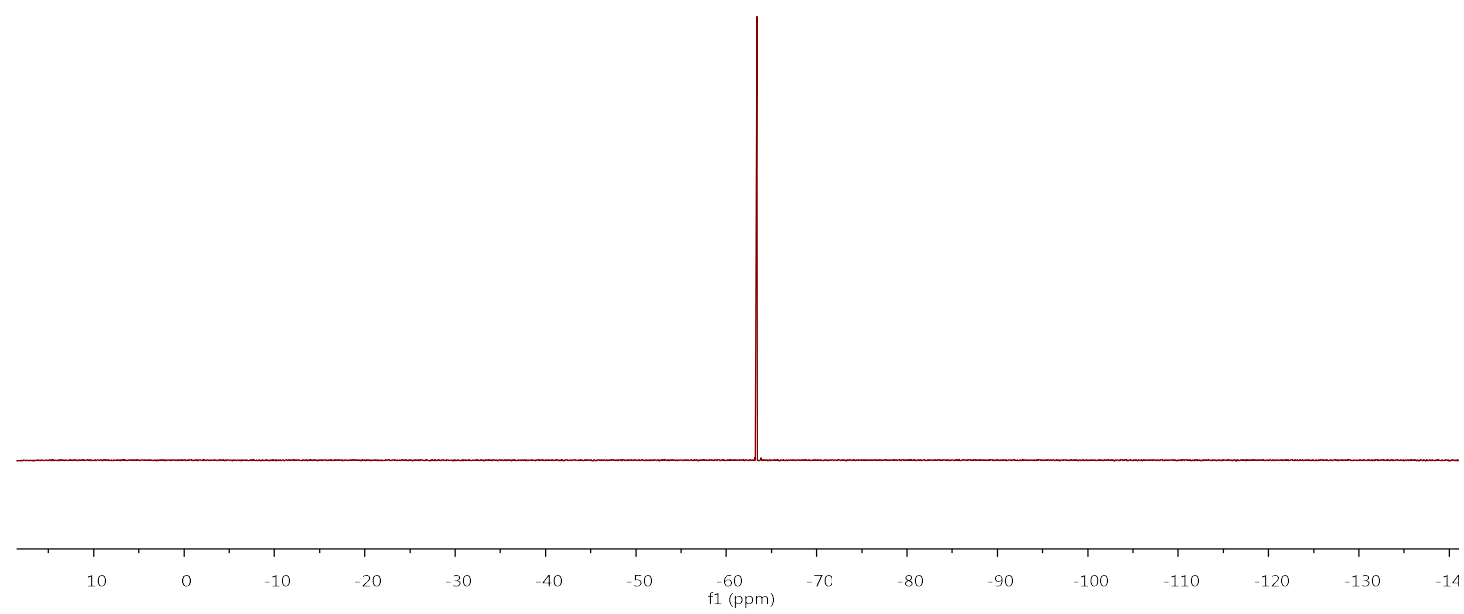




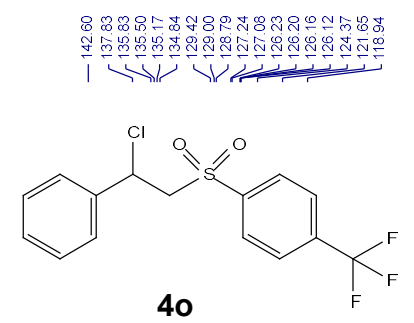

${ }^{13} \mathrm{C}$ NMR (101 MHz, $\left.\mathrm{CDCl}_{3}\right)$

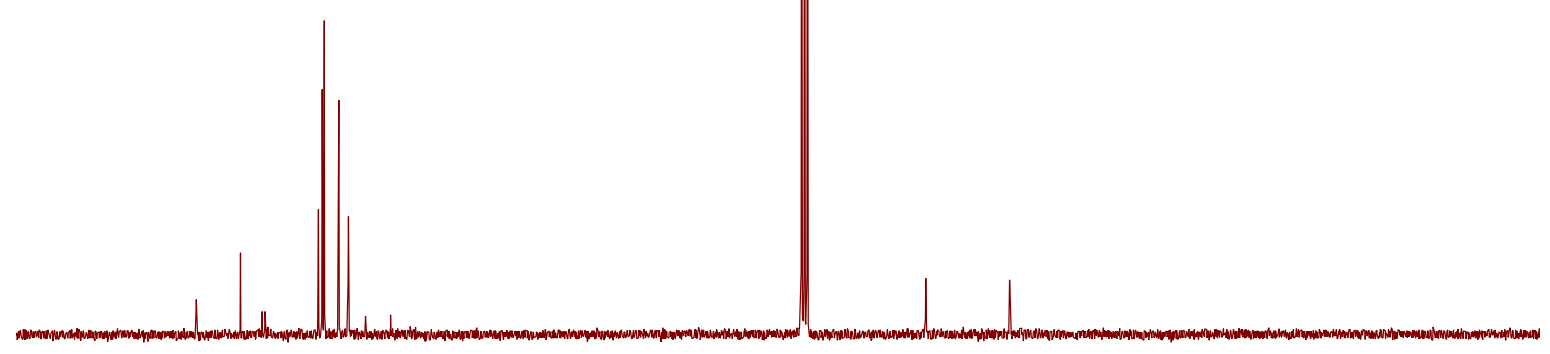

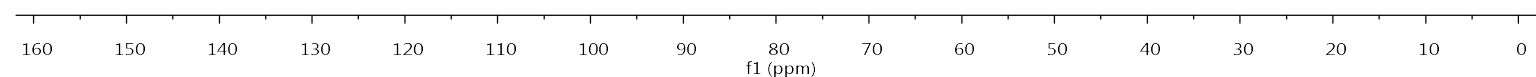


(S)-4-((2-chloro-2-phenylethyl)sulfonyl)benzonitrile

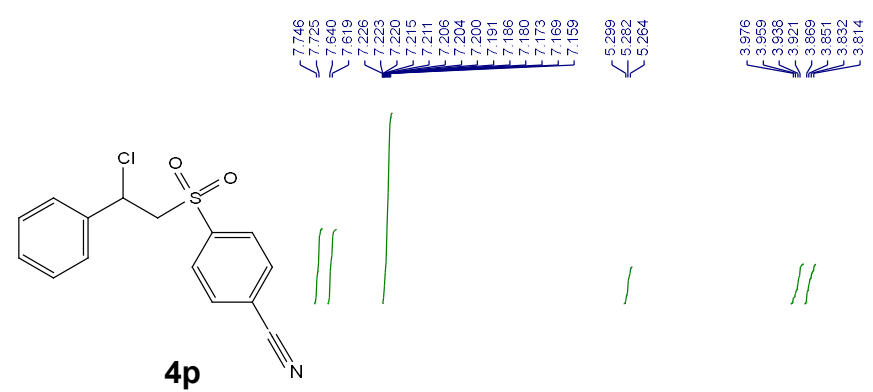

${ }^{1} \mathrm{H}$ NMR $\left(400 \mathrm{MHz}, \mathrm{CDCl}_{3}\right)$

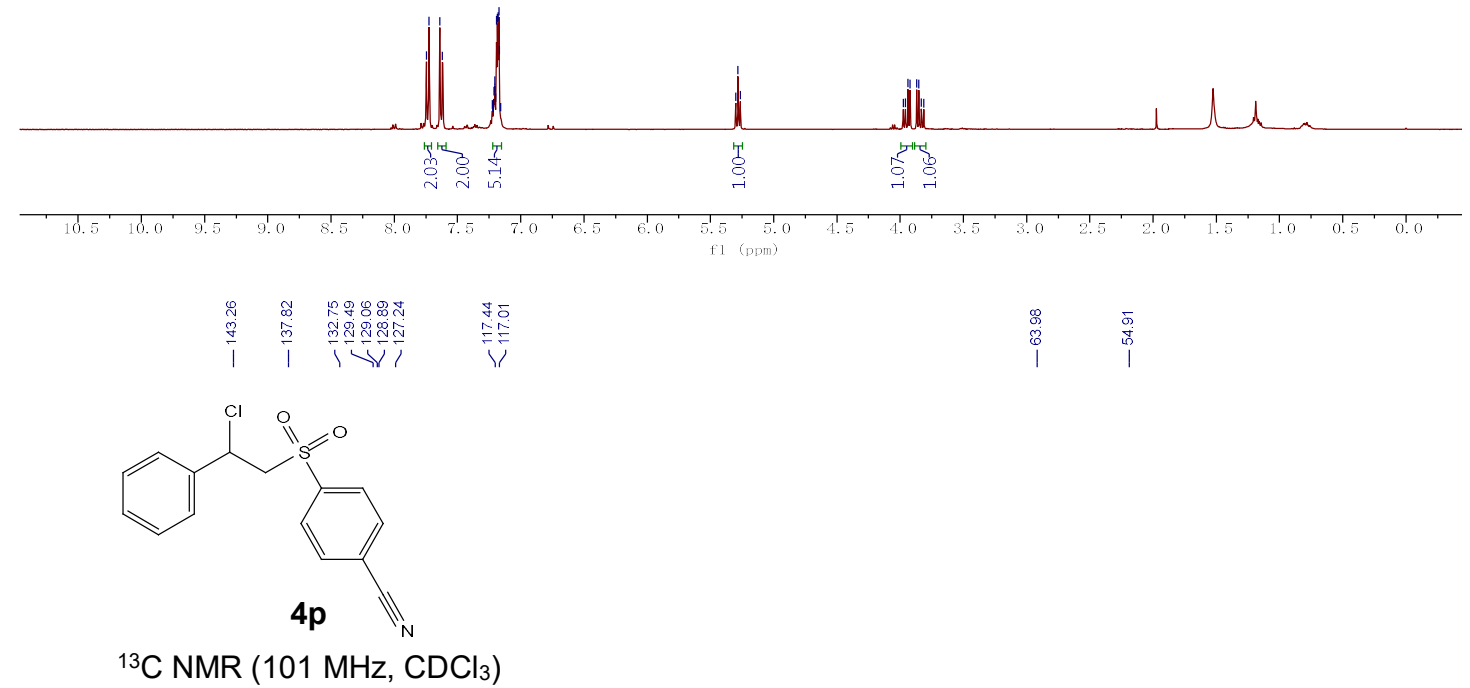

${ }^{13} \mathrm{C}$ NMR (101 MHz, $\left.\mathrm{CDCl}_{3}\right)$

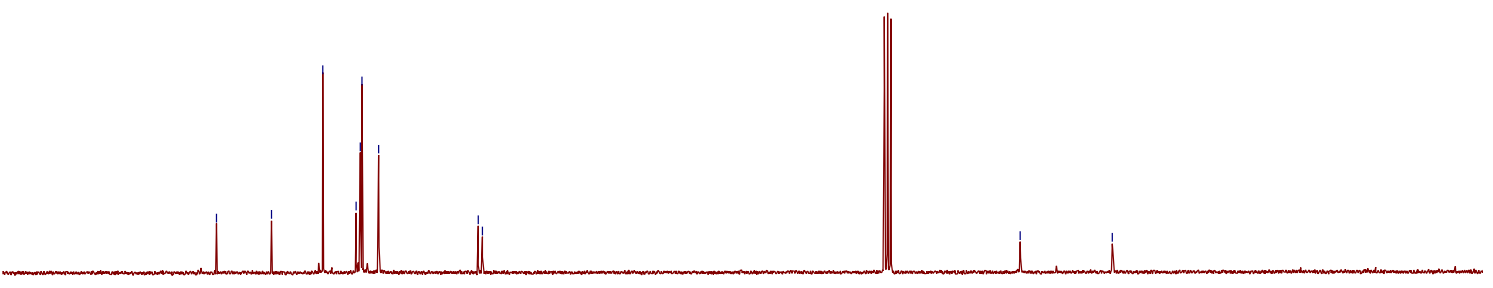


(2S,5S)-5-isopropyl-2-methylcyclohexyl 4-(((R)-2-chloro-2-(4-

fluorophenyl)ethyl)sulfonyl)benzoate

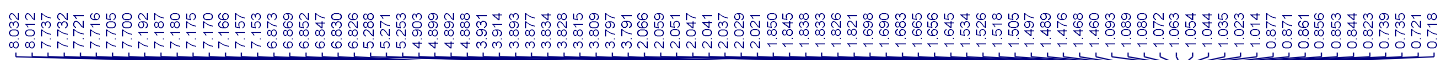
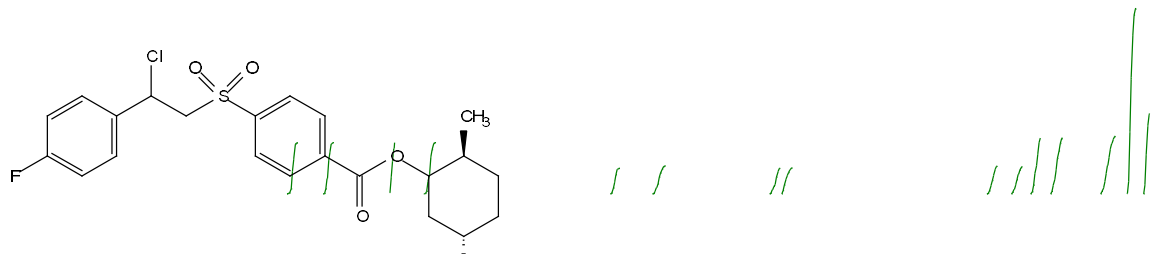

$4 q$

${ }^{1} \mathrm{H}$ NMR $\left(400 \mathrm{MHz}, \mathrm{CDCl}_{3}\right)$
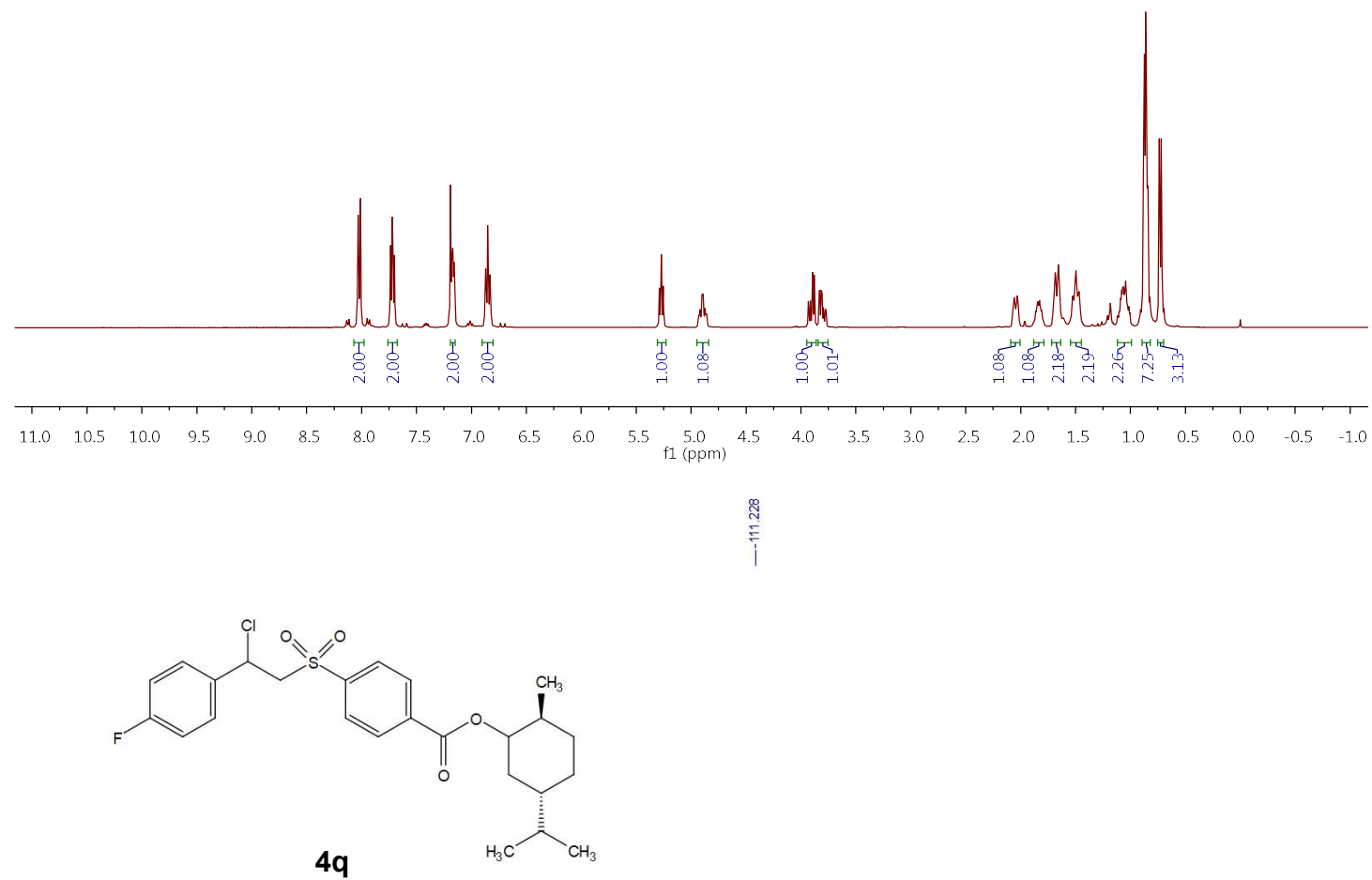

${ }^{19} \mathrm{~F}$ NMR $\left(376 \mathrm{MHz}, \mathrm{CDCl}_{3}\right)$

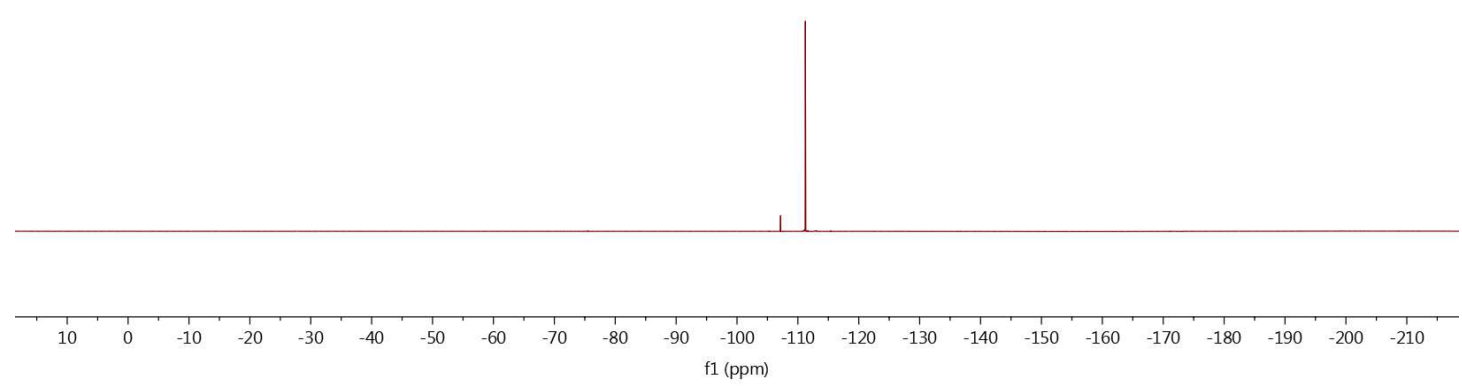



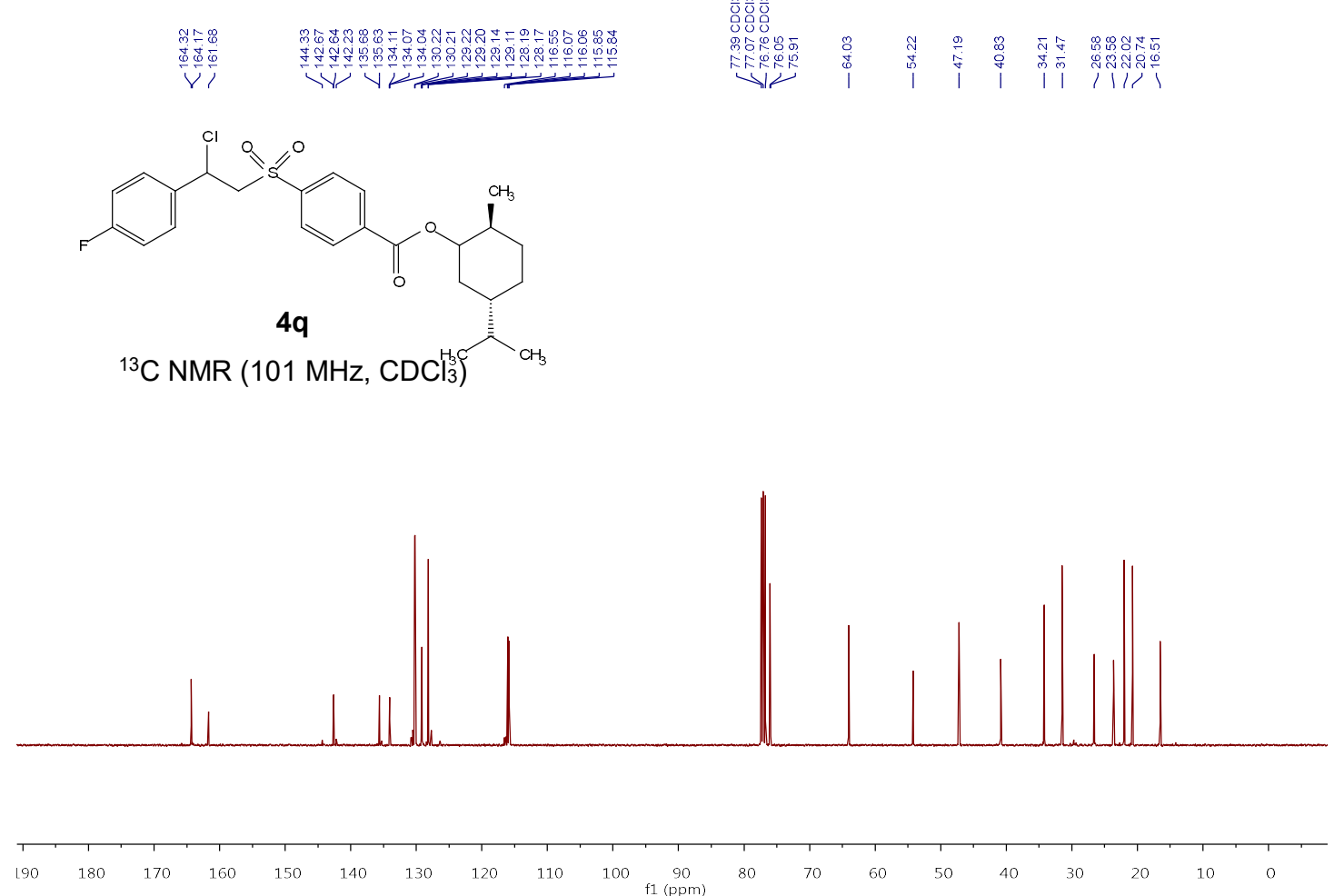
(S)-4-(1-chloro-2-((4-methoxyphenyl)sulfonyl)ethyl)-1,1'-biphenyl

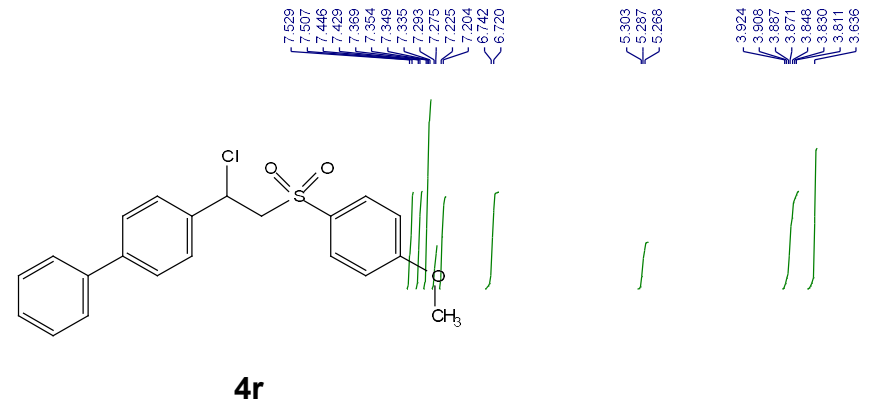

${ }^{1} \mathrm{H}$ NMR $\left(400 \mathrm{MHz}, \mathrm{CDCl}_{3}\right)$

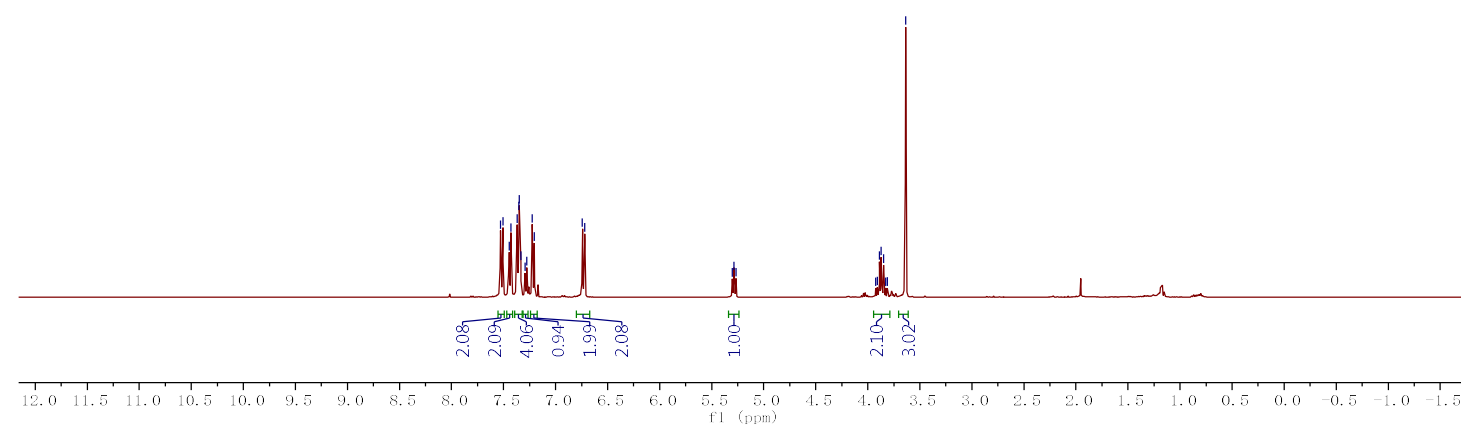

$\begin{array}{llll}1 & 11 \\ 8 & 1\end{array}$

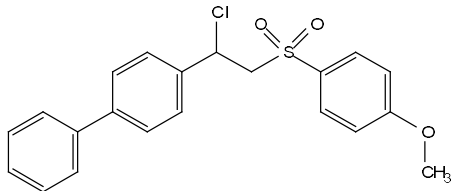

4

${ }^{13} \mathrm{C} \mathrm{NMR}\left(101 \mathrm{MHz}, \mathrm{CDCl}_{3}\right)$

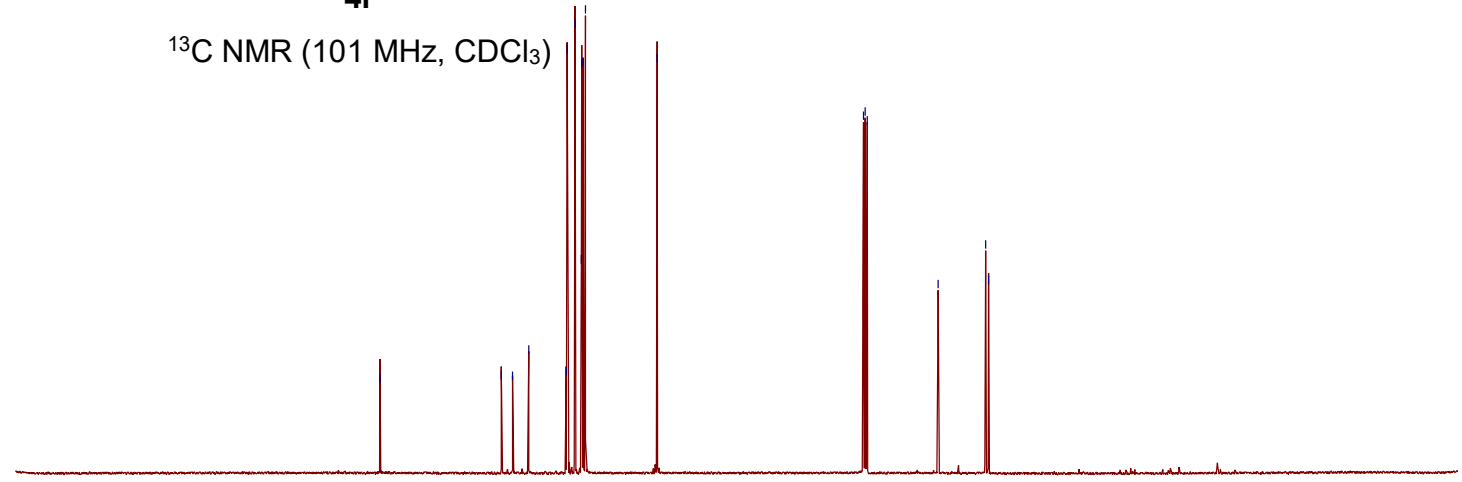


(S)-2-(1-chloro-2-((4-methoxyphenyl)sulfonyl)ethyl)naphthalene

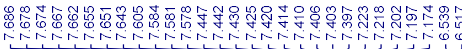

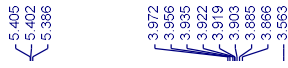

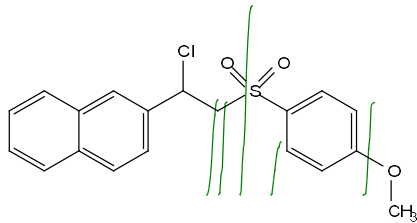

$4 \mathbf{s}$

${ }^{1} \mathrm{H}$ NMR $\left(400 \mathrm{MHz}, \mathrm{CDCl}_{3}\right)$
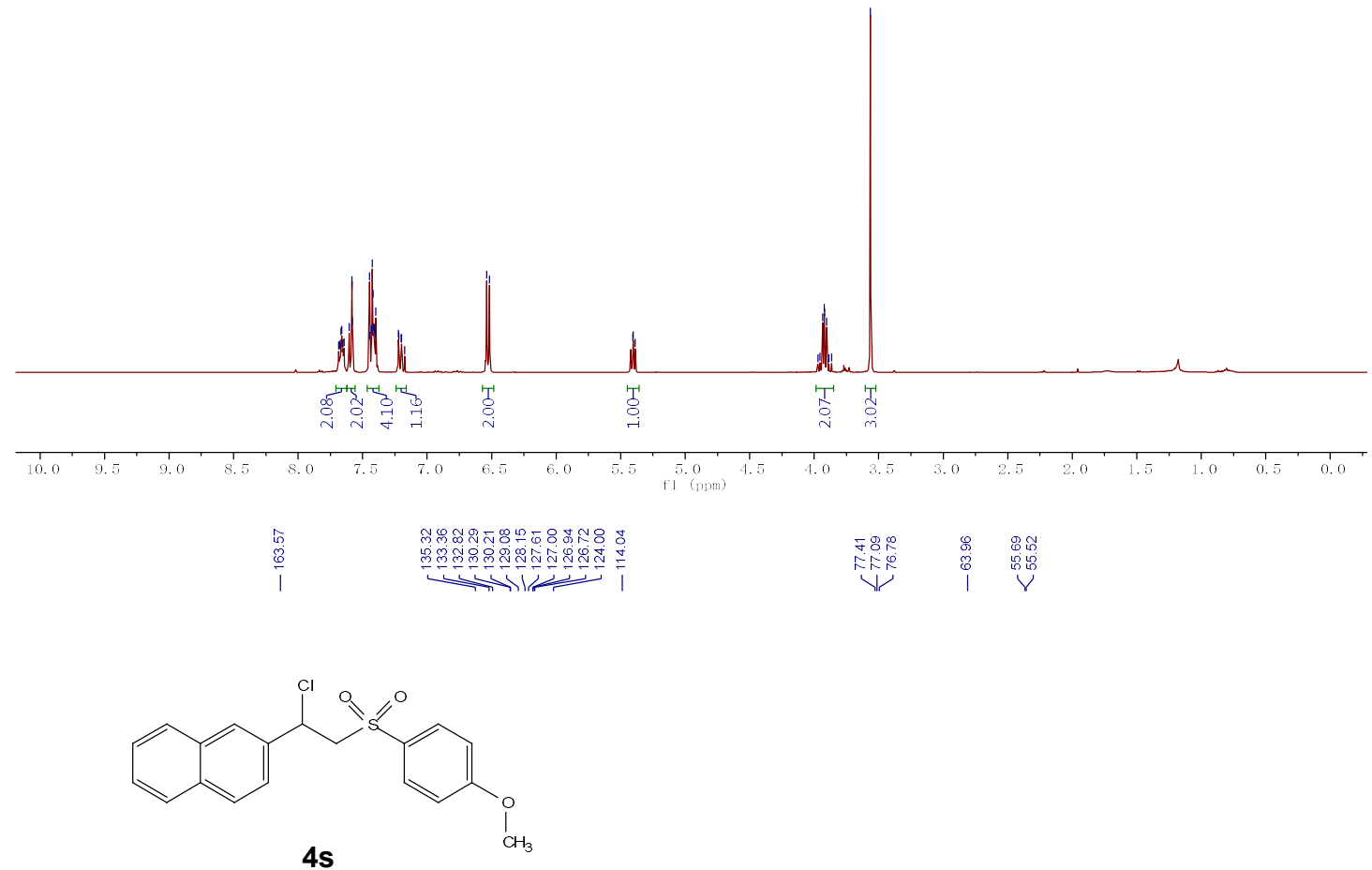

${ }^{13} \mathrm{C}$ NMR $\left(101 \mathrm{MHz}, \mathrm{CDCl}_{3}\right)$

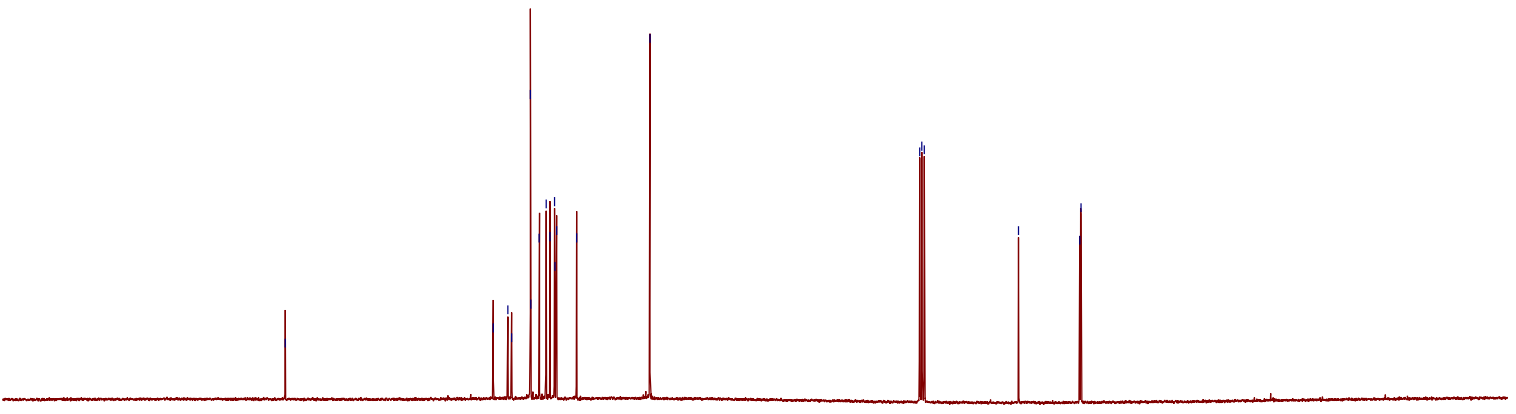


(R)-2-(1-chloro-2-((4-phenoxyphenyl)sulfonyl)ethyl)-1,4-dimethylbenzene

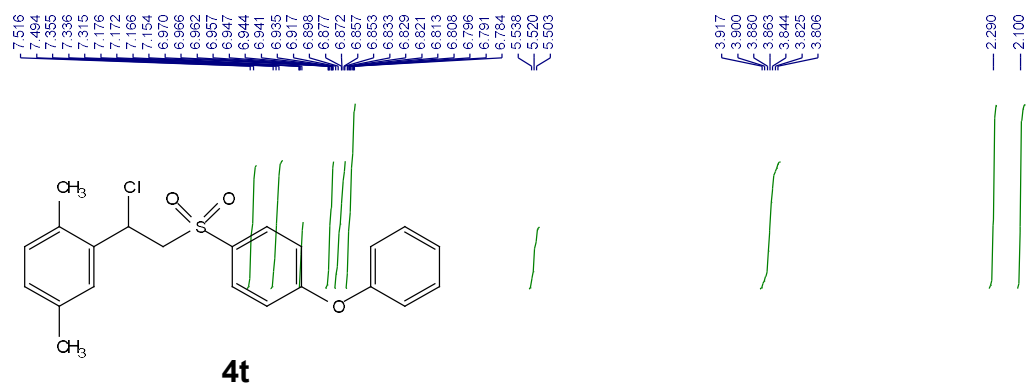

${ }^{1} \mathrm{H}$ NMR $\left(400 \mathrm{MHz}, \mathrm{CDCl}_{3}\right)$
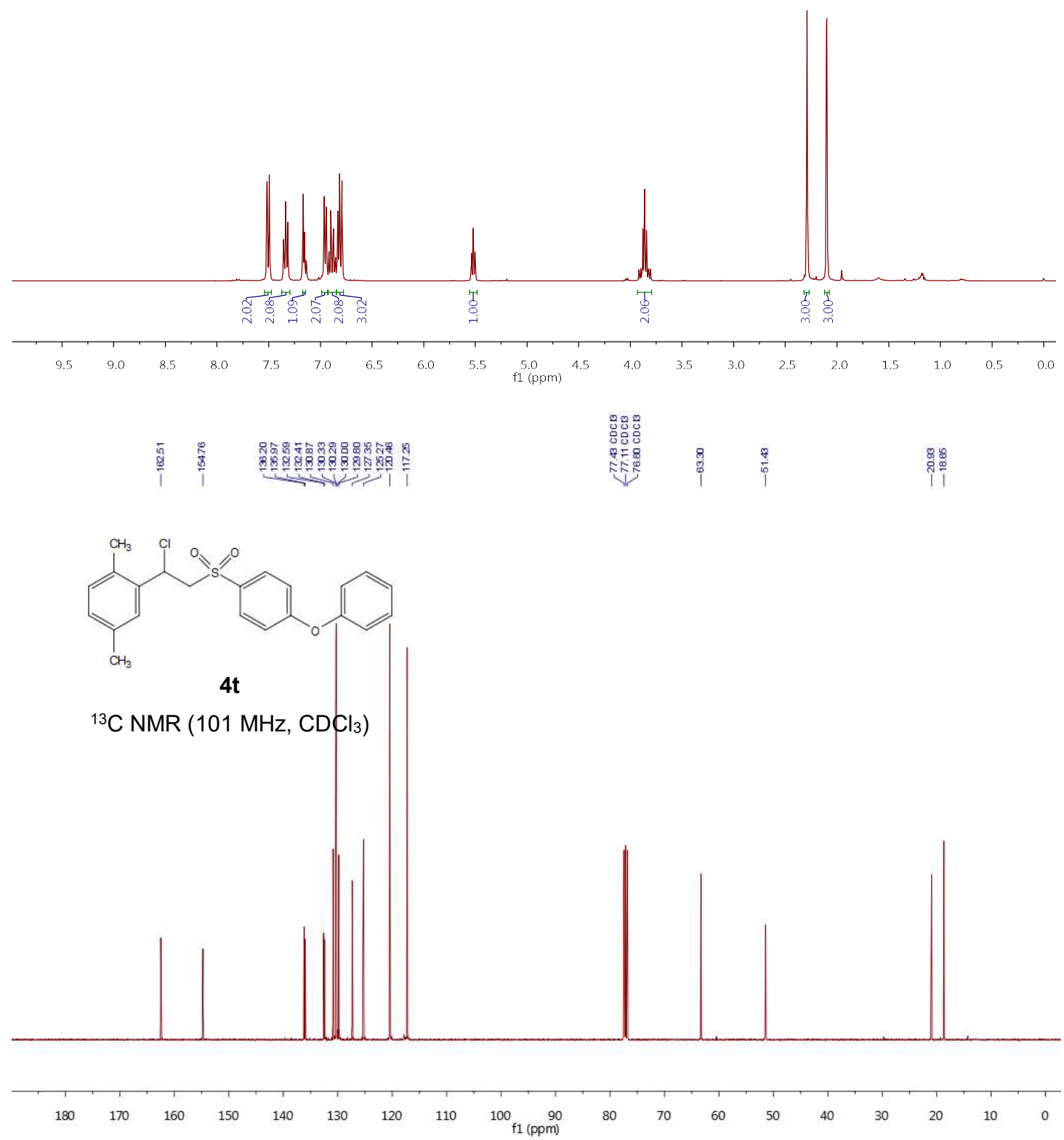
(R)-1-(tert-butyl)-4-(1-chloro-2-((4-methoxyphenyl)sulfonyl)ethyl)benzene
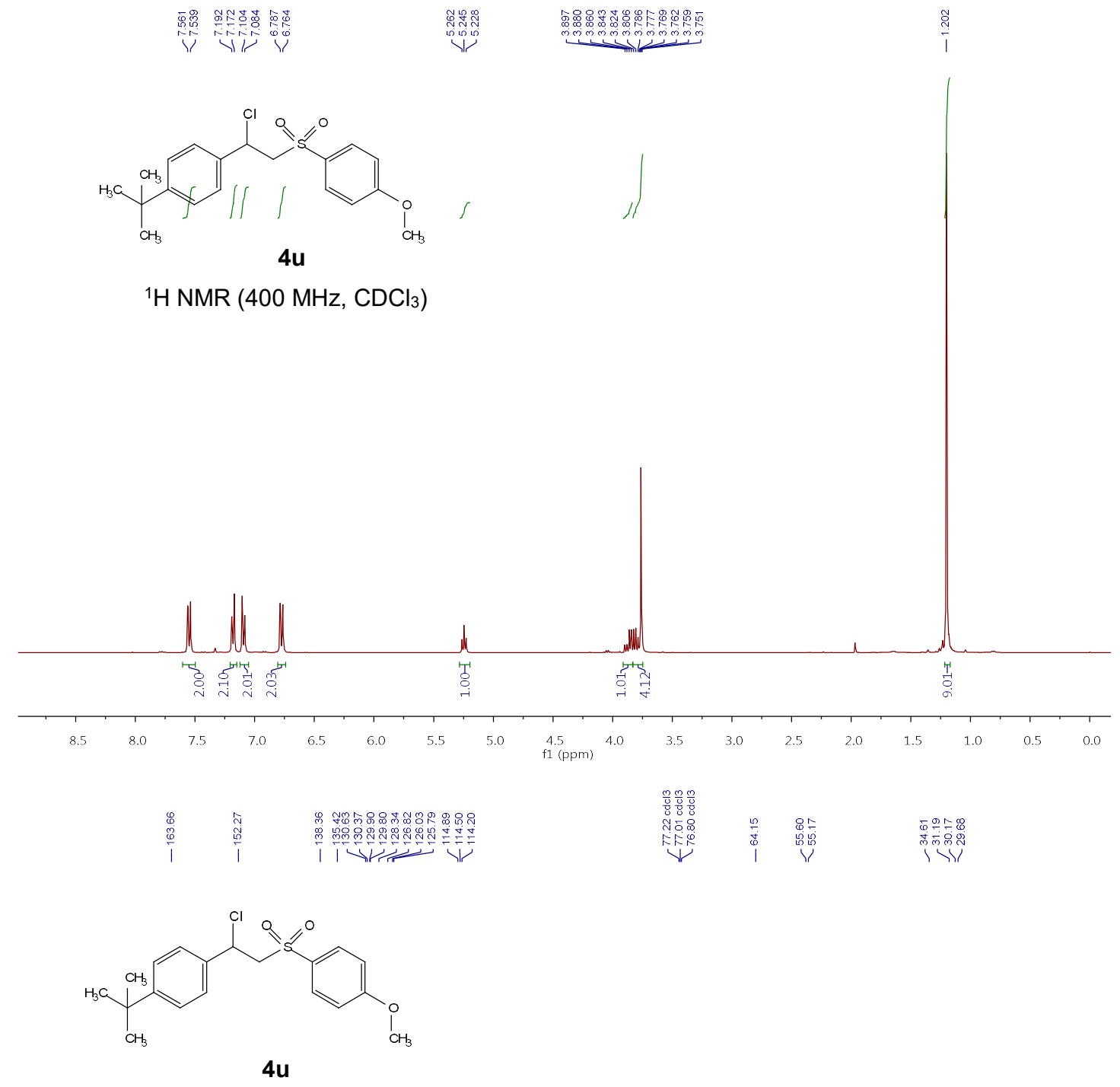

${ }^{13} \mathrm{C} \mathrm{NMR}\left(101 \mathrm{MHz}, \mathrm{CDCl}_{3}\right)$

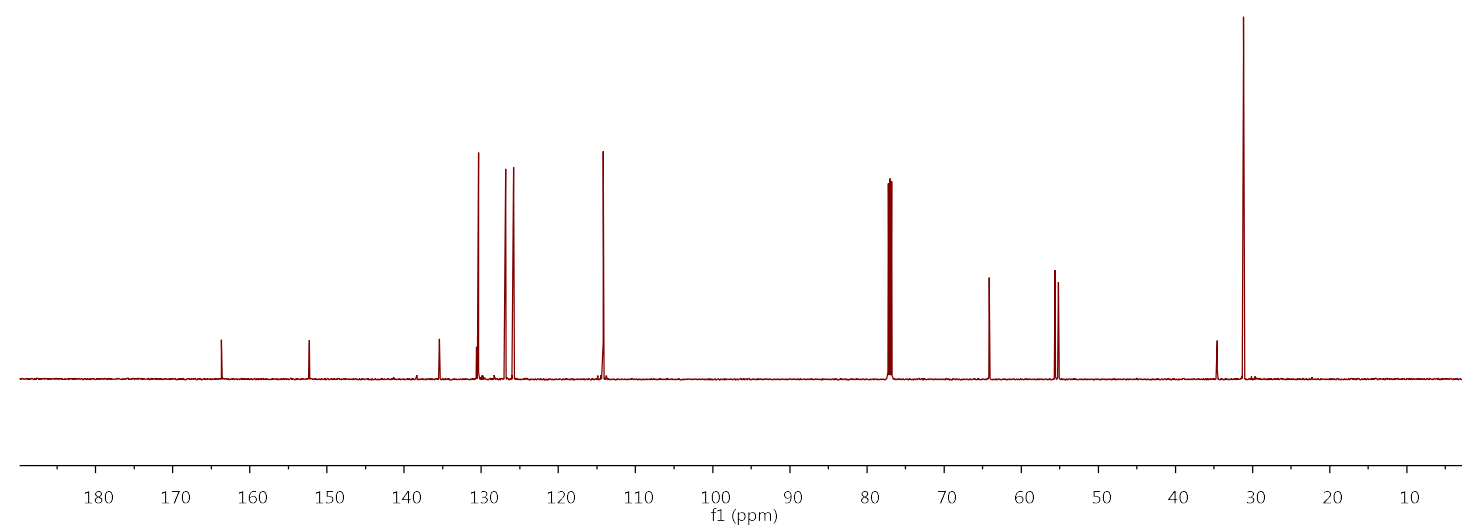


(S)-1-(1-chloro-2-((4-methoxyphenyl)sulfonyl)ethyl)-2-methoxybenzene

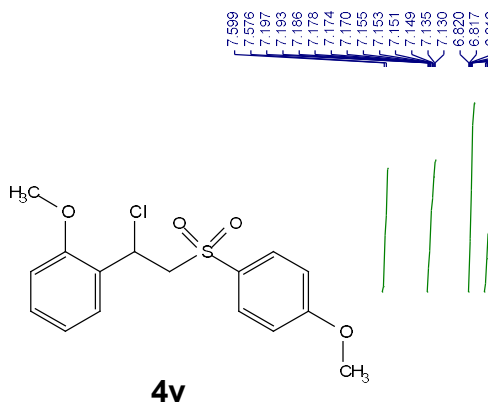

${ }^{1} \mathrm{H} \mathrm{NMR}\left(400 \mathrm{MHz}, \mathrm{CDCl}_{3}\right)$
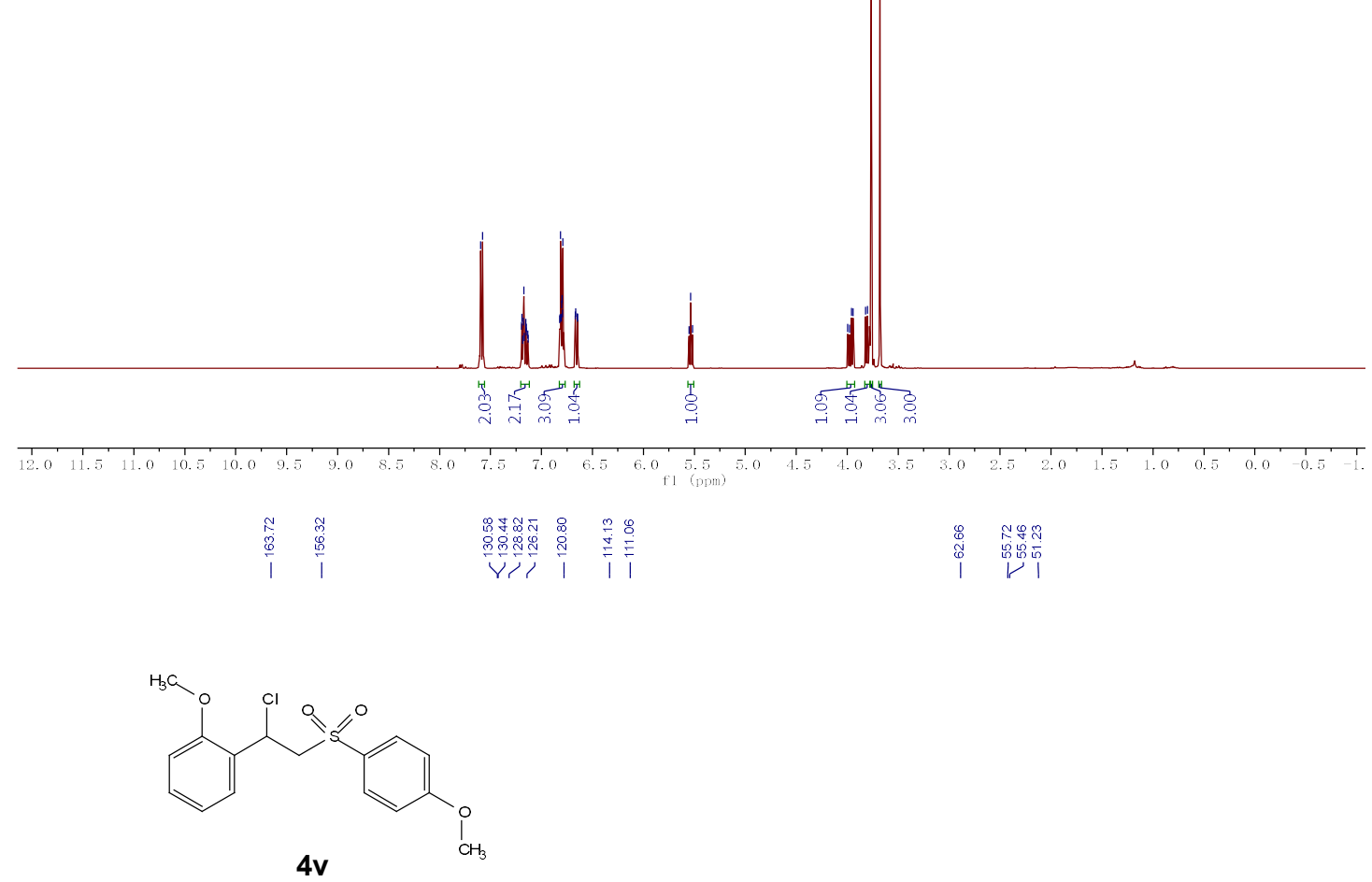

${ }^{13} \mathrm{C}$ NMR $\left(101 \mathrm{MHz}, \mathrm{CDCl}_{3}\right)$

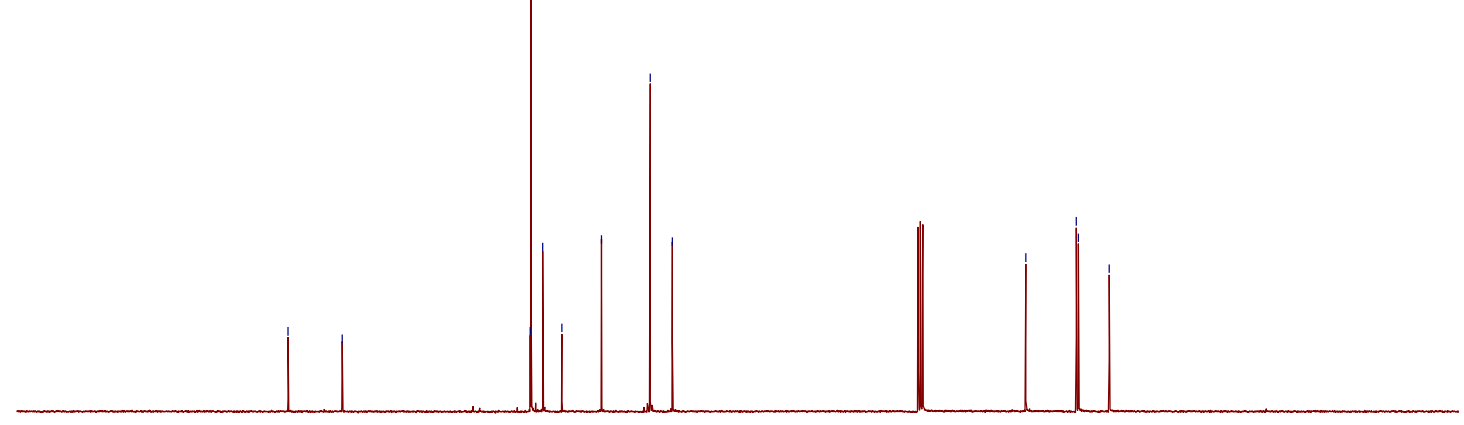

200

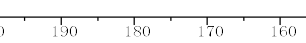


(S)-1-(1-chloro-2-((4-methoxyphenyl)sulfonyl)ethyl)-4-(trifluoromethyl)benzene

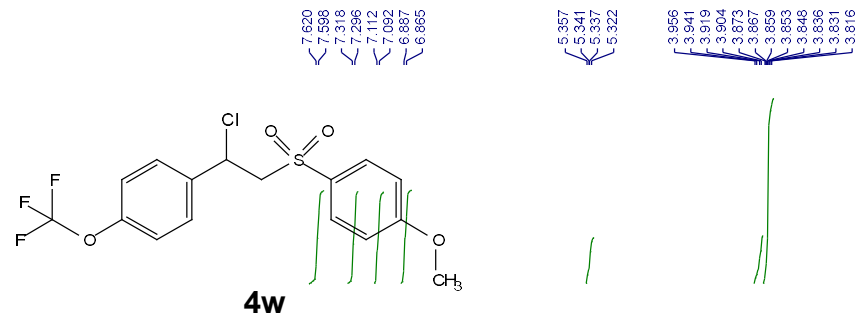

${ }^{1} \mathrm{H}$ NMR (400 MHz, $\left.\mathrm{CDCl}_{3}\right)$
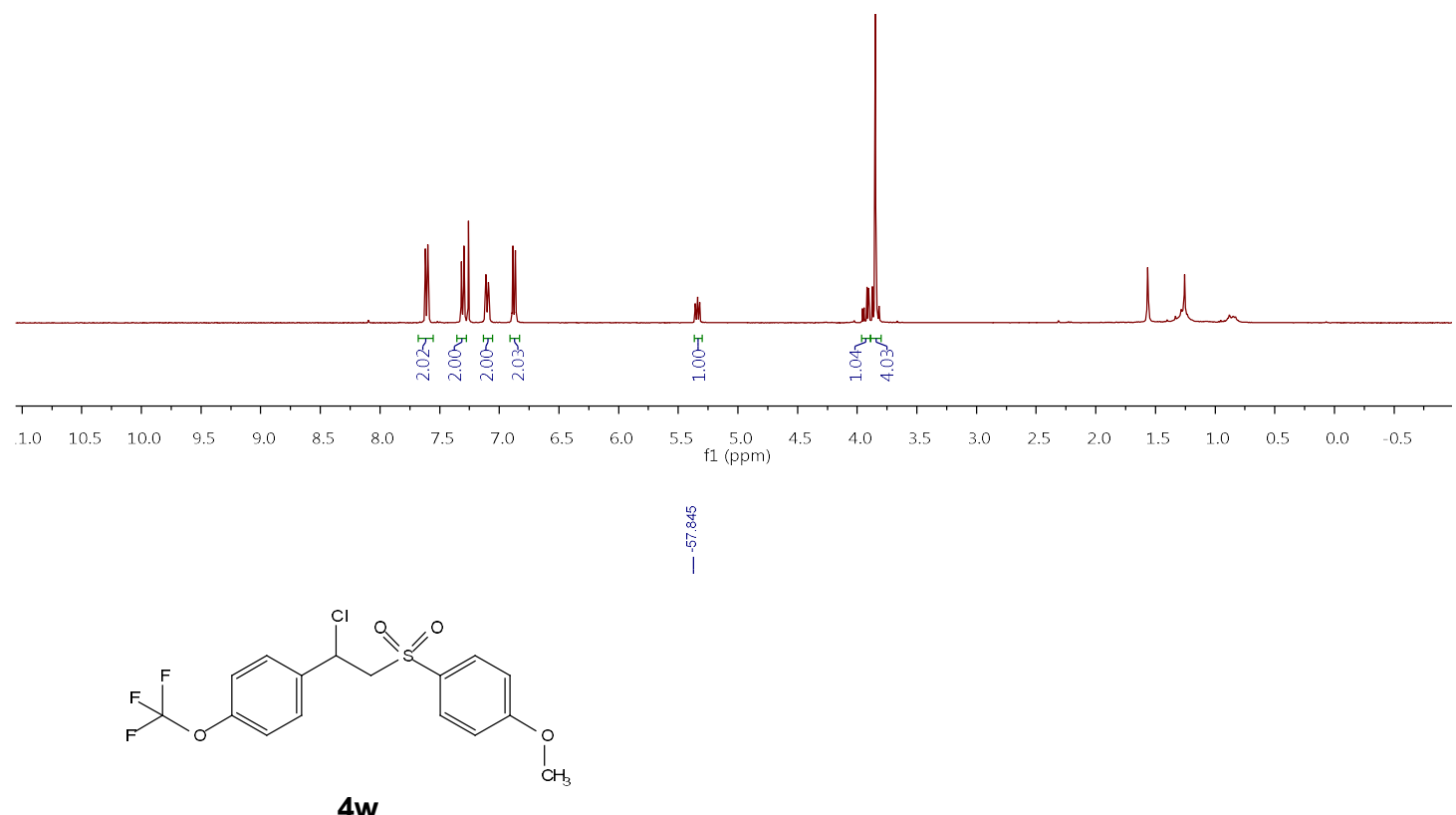

${ }^{19} \mathrm{~F} \mathrm{NMR}\left(376 \mathrm{MHz}, \mathrm{CDCl}_{3}\right)$

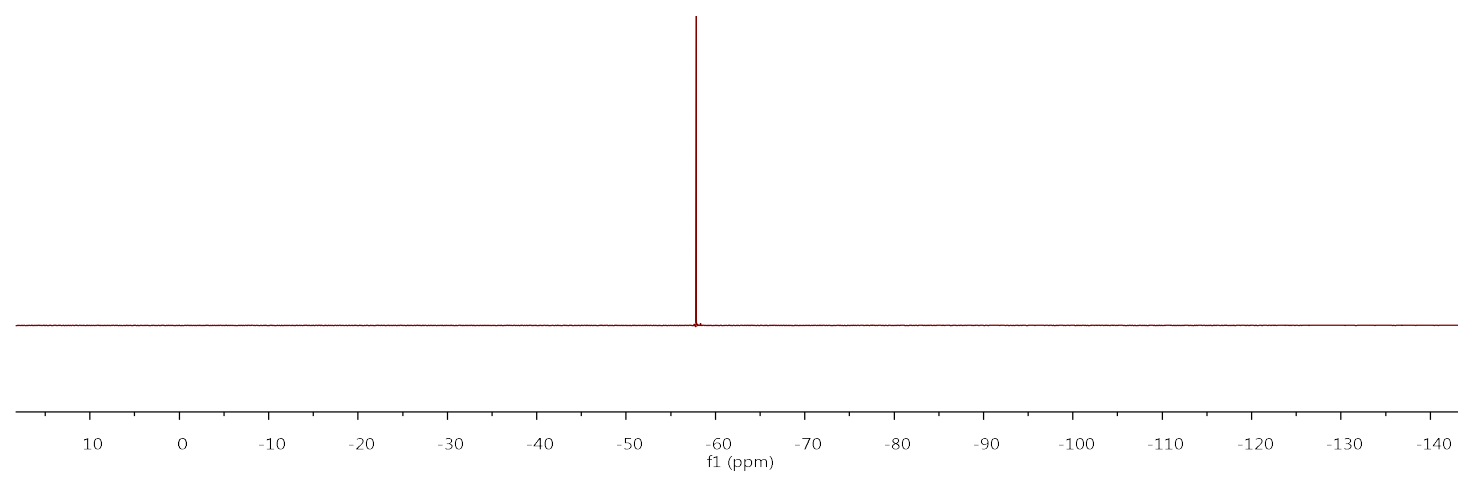




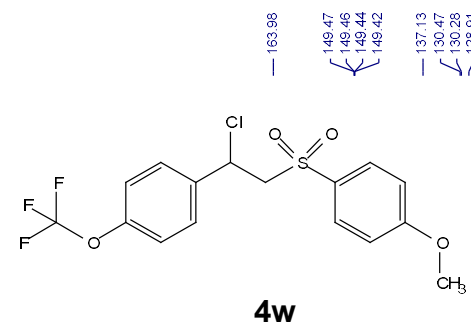

$4 w$

${ }^{13} \mathrm{C}$ NMR (101 MHz, CDCl 3 )
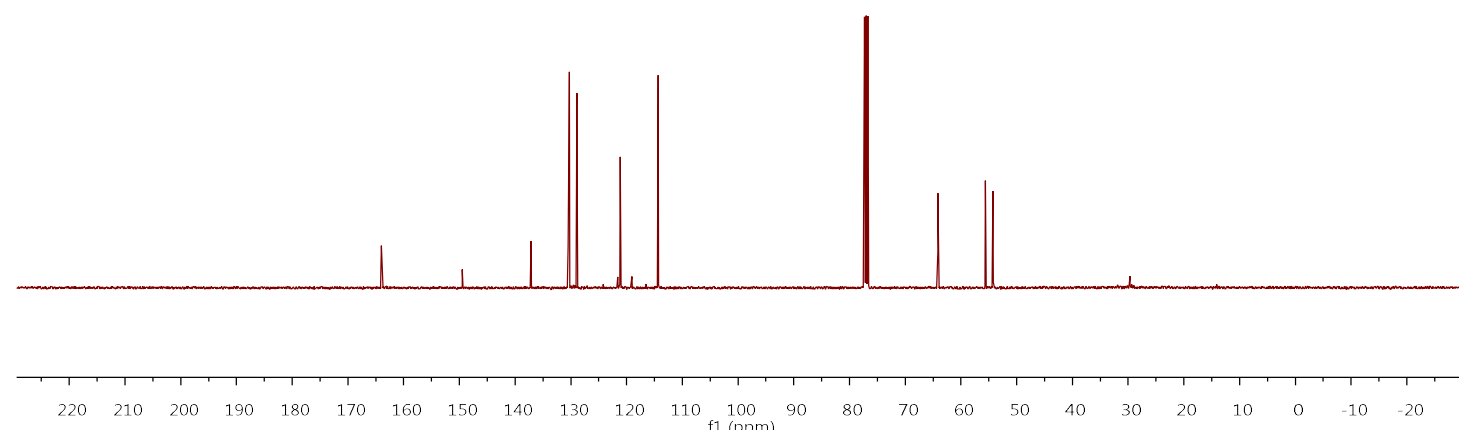
(S)-1-(1-chloro-2-((4-methoxyphenyl)sulfonyl)ethyl)-4-fluorobenzene
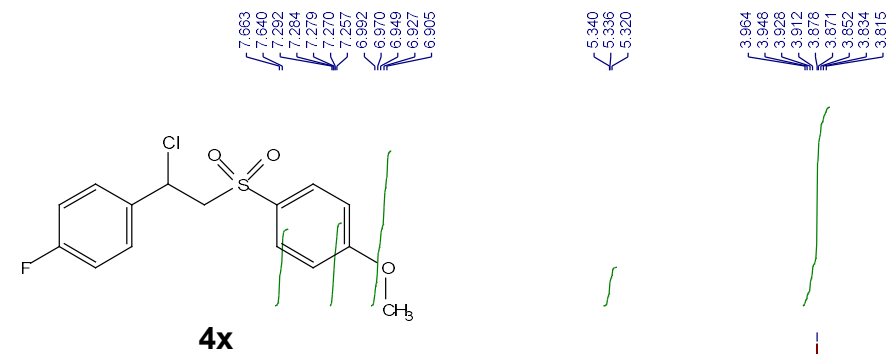

${ }^{1} \mathrm{H} \mathrm{NMR}\left(400 \mathrm{MHz}, \mathrm{CDCl}_{3}\right)$
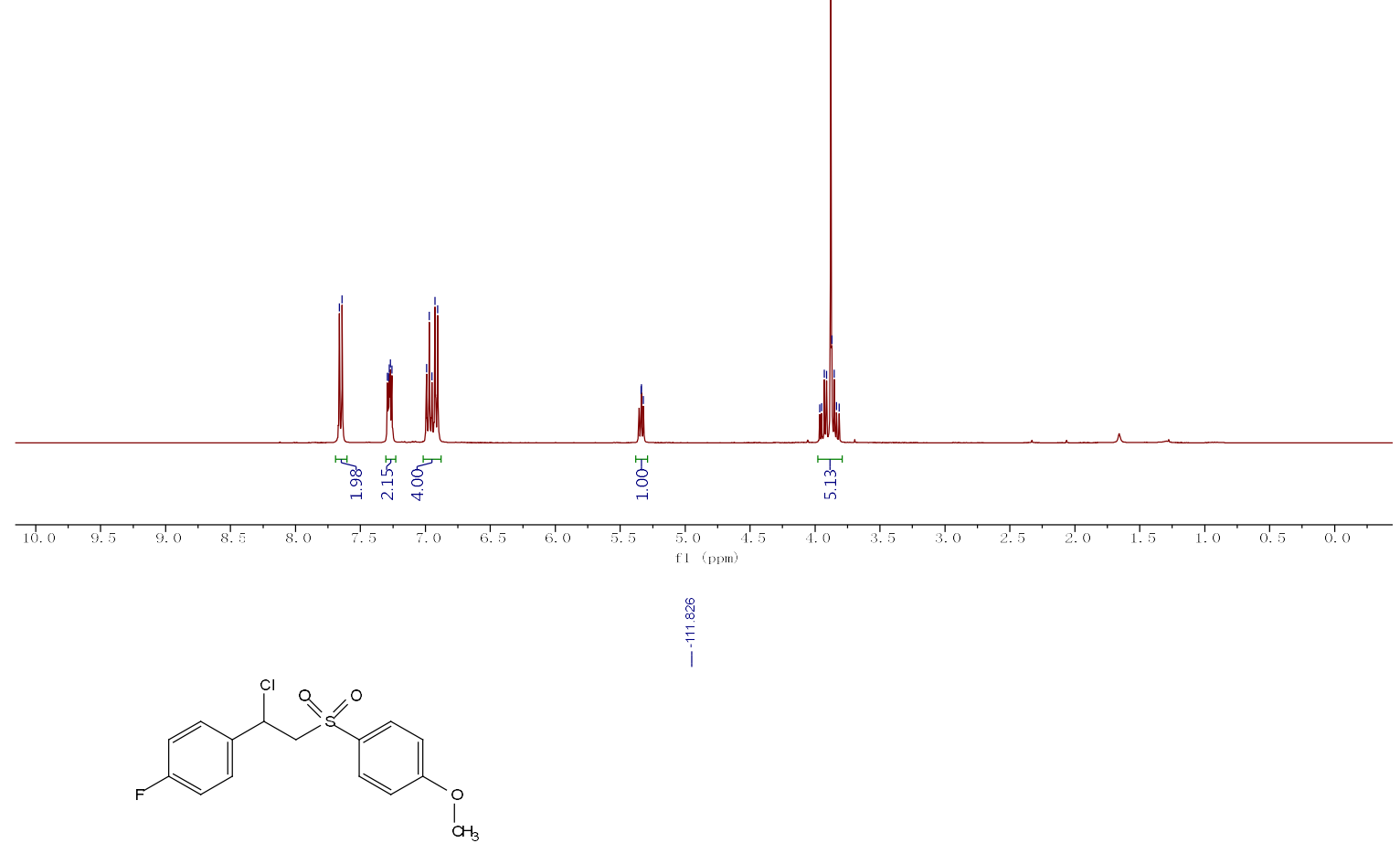

$4 \mathbf{x}$

${ }^{19} \mathrm{~F} \mathrm{NMR}\left(376 \mathrm{MHz}, \mathrm{CDCl}_{3}\right)$

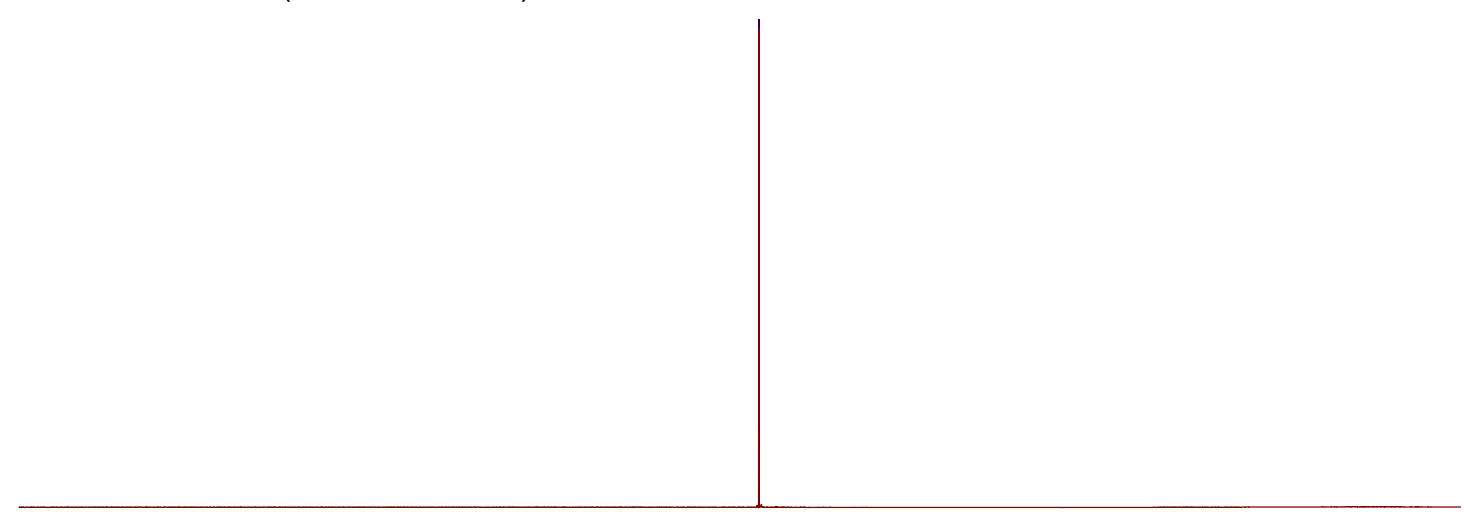




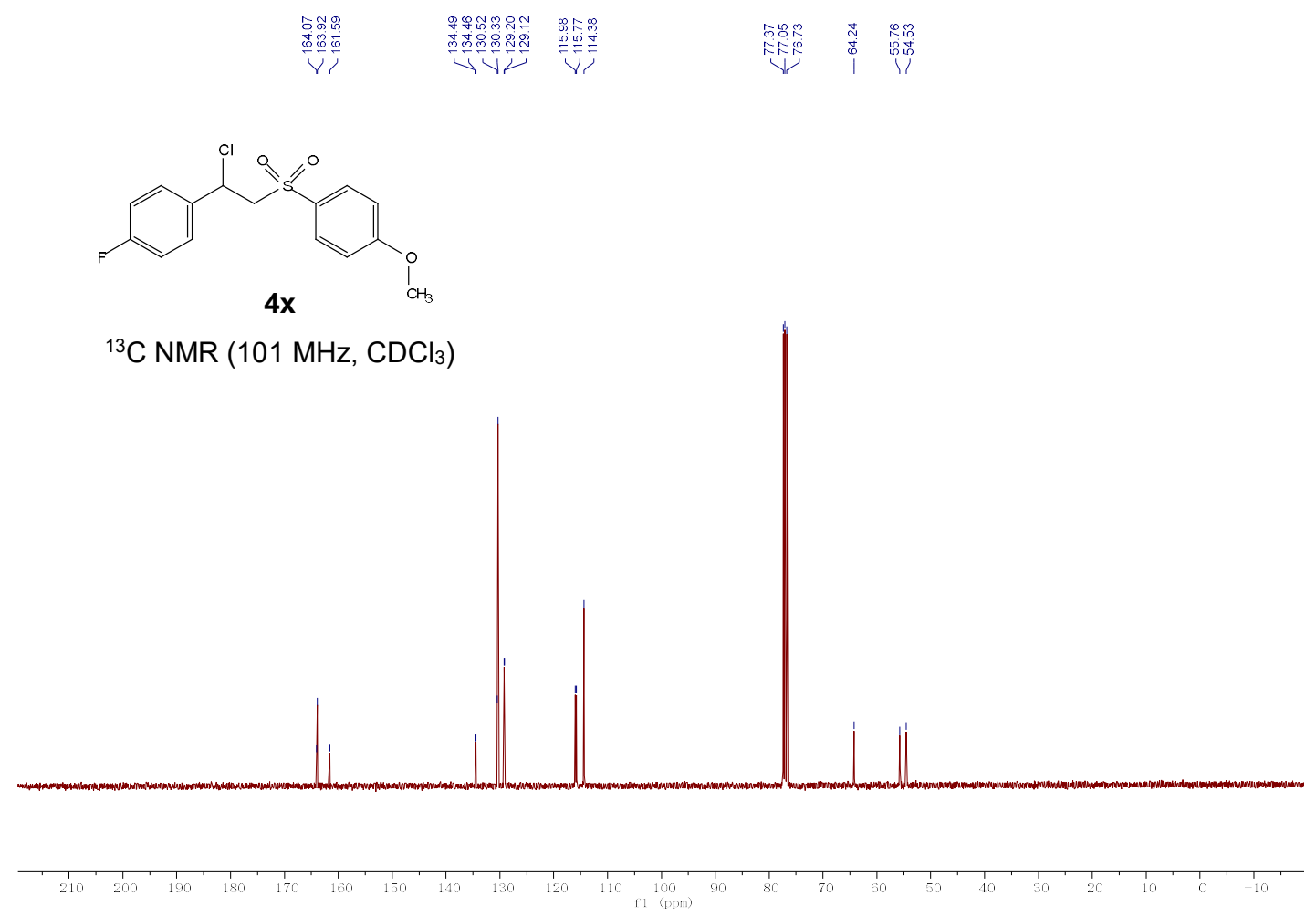


(R)-1-chloro-4-(1-chloro-2-((4-methoxyphenyl)sulfonyl)ethyl)benzene
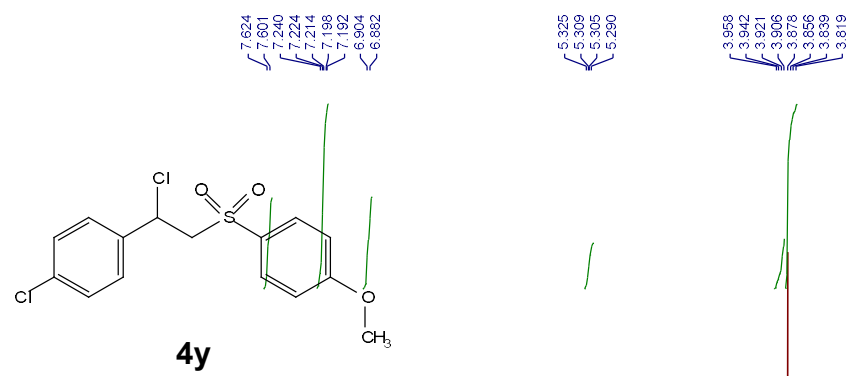

${ }^{1} \mathrm{H}$ NMR $\left(400 \mathrm{MHz}, \mathrm{CDCl}_{3}\right)$
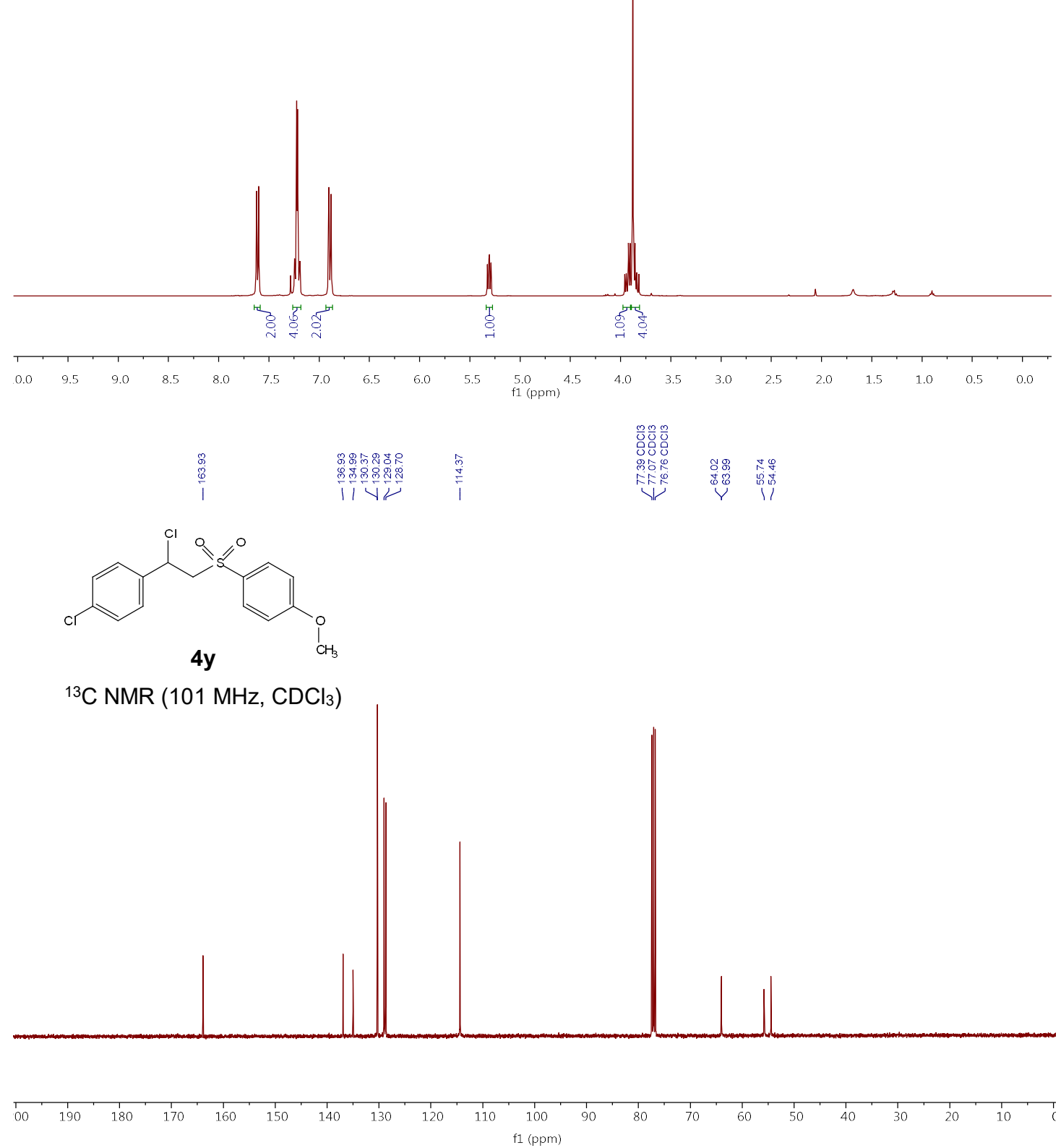
(R)-1-(2-((4-(tert-butyl)phenyl)sulfonyl)-1-chloroethyl)-3-(chloromethyl)benzene
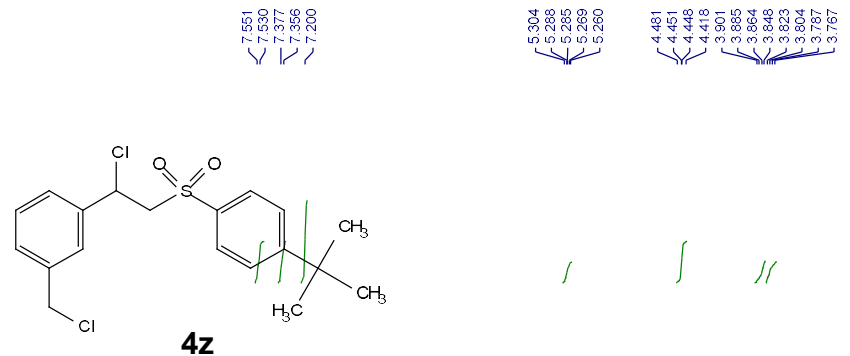

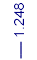

${ }^{1} \mathrm{H} \mathrm{NMR}\left(400 \mathrm{MHz}, \mathrm{CDCl}_{3}\right)$
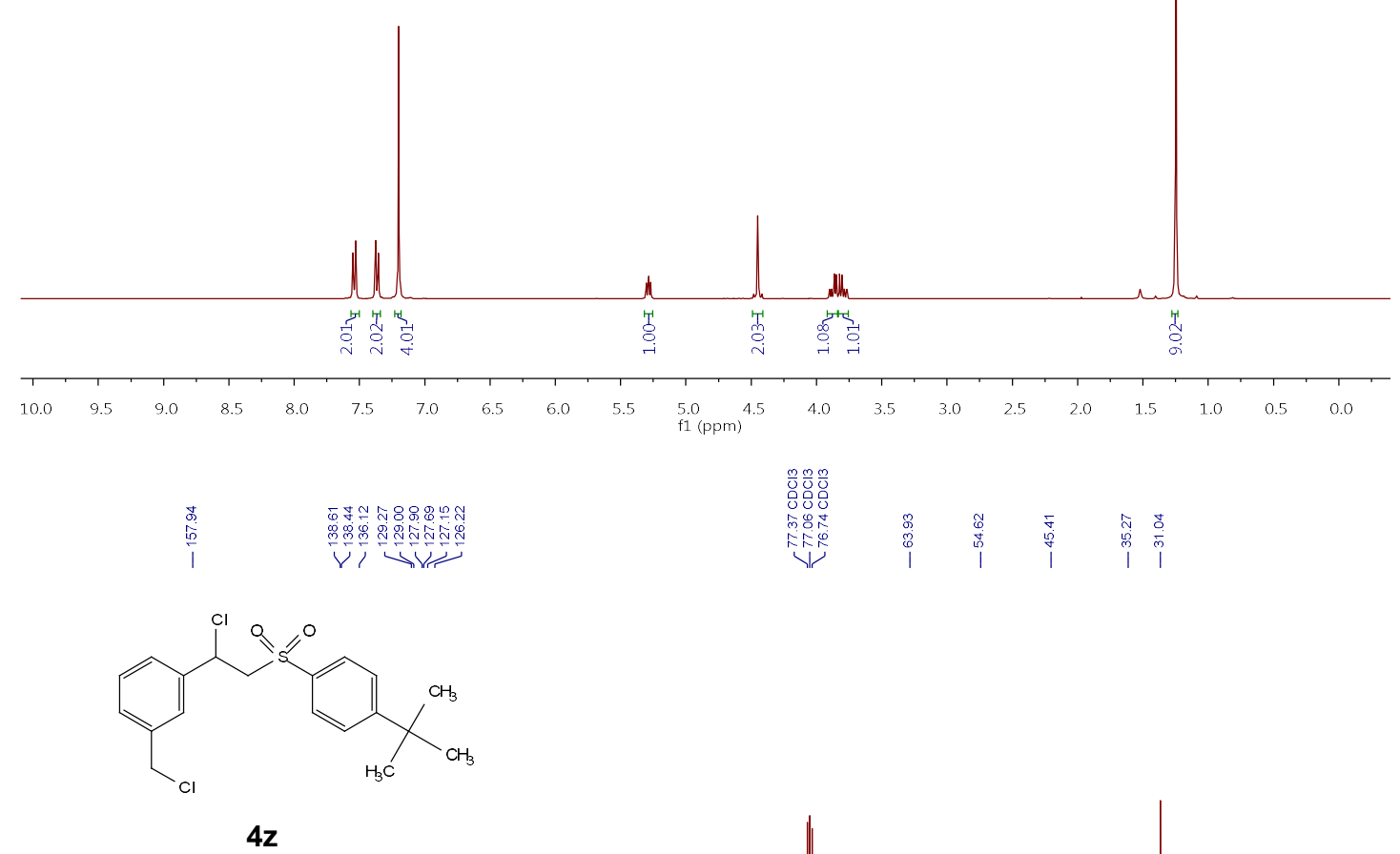

${ }^{13} \mathrm{C}$ NMR $\left(101 \mathrm{MHz}, \mathrm{CDCl}_{3}\right)$
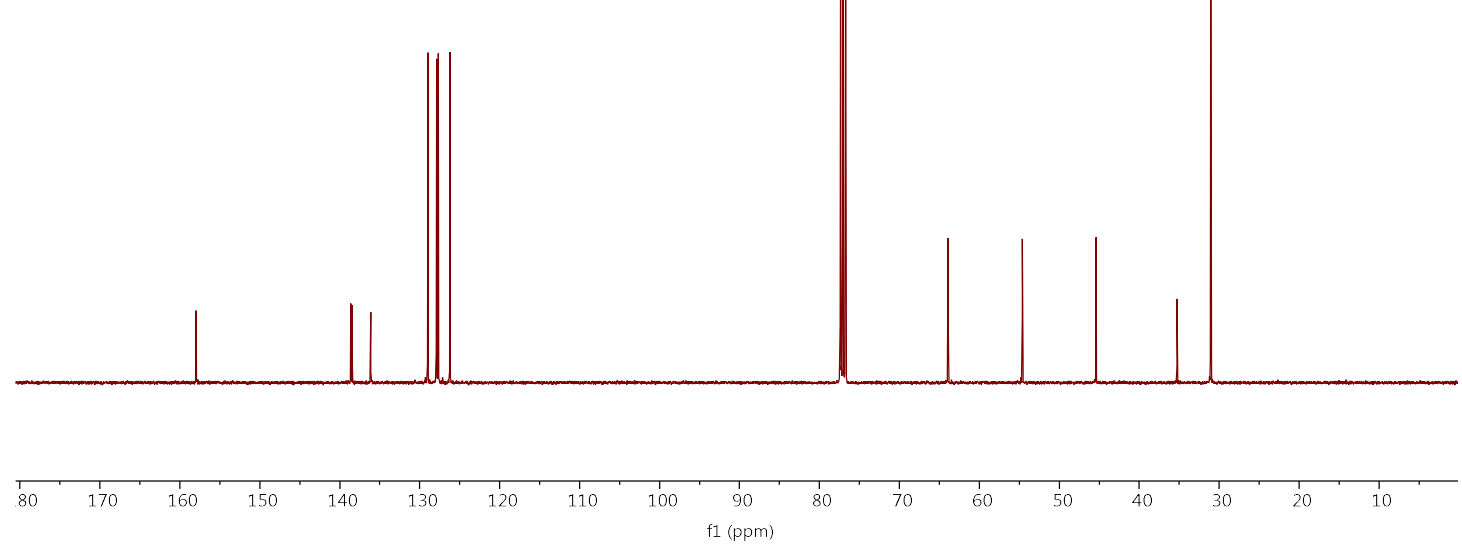
(S)-1-bromo-2-(1-chloro-2-((4-methoxyphenyl)sulfonyl)ethyl)benzene

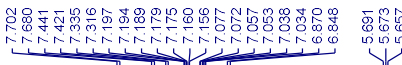

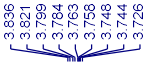

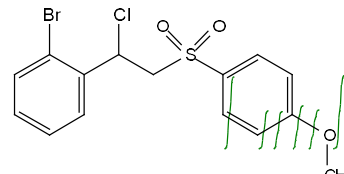

4aa

\author{
$\mathrm{CH}_{3}$
}

${ }^{1} \mathrm{H}$ NMR $\left(400 \mathrm{MHz}, \mathrm{CDCl}_{3}\right)$

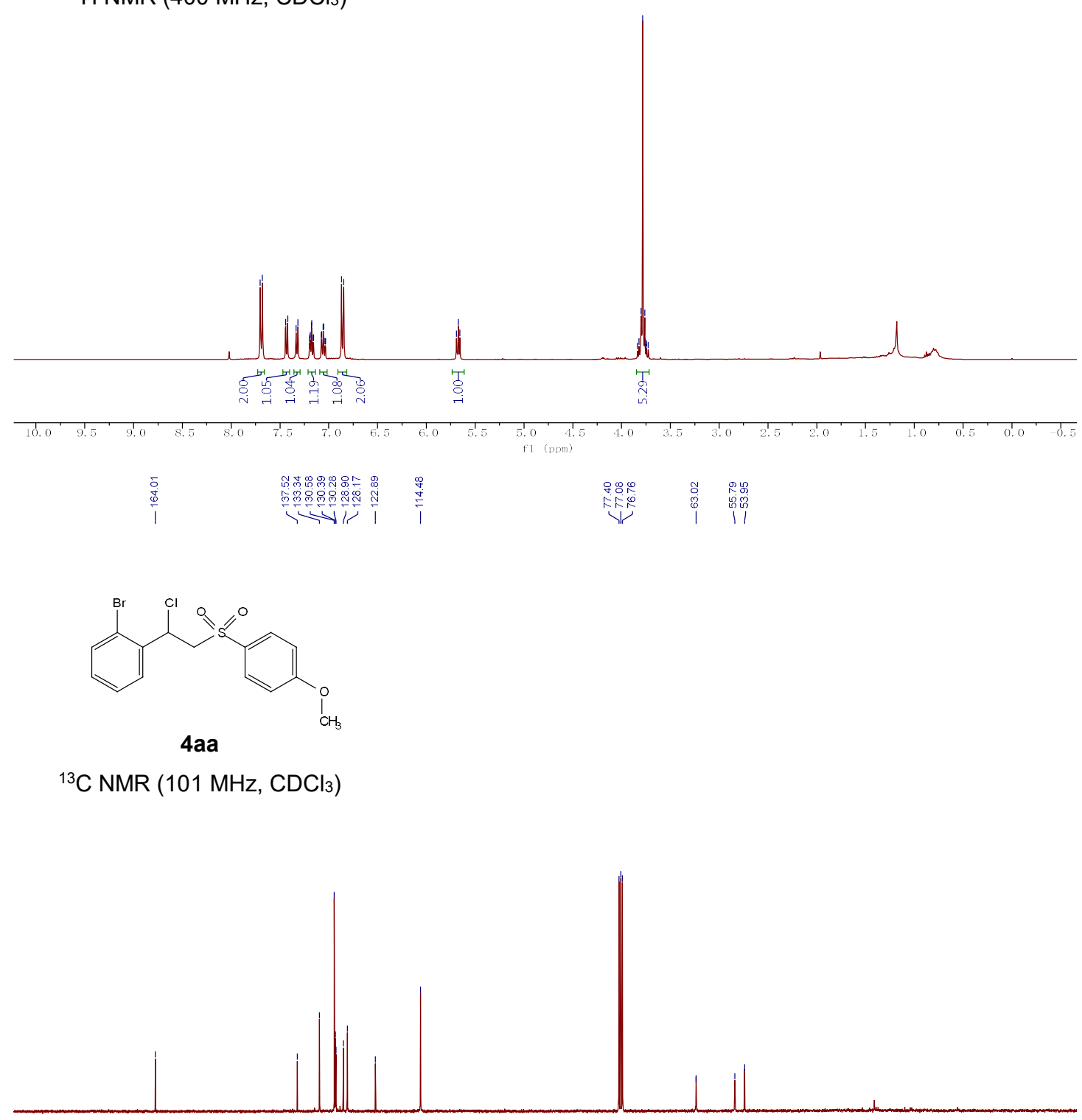

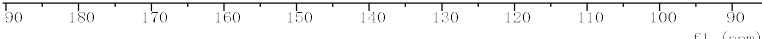


(S)-4-(1-chloro-2-((4-methoxyphenyl)sulfonyl)ethyl)benzonitrile
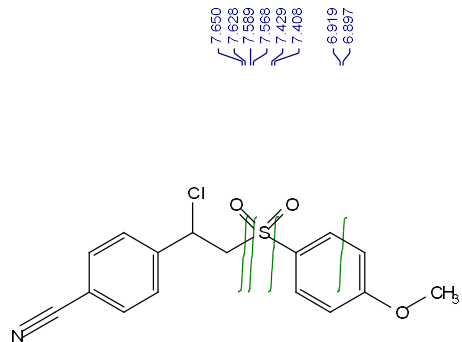

$4 a b$

${ }^{1} \mathrm{H}$ NMR $\left(400 \mathrm{MHz}, \mathrm{CDCl}_{3}\right)$
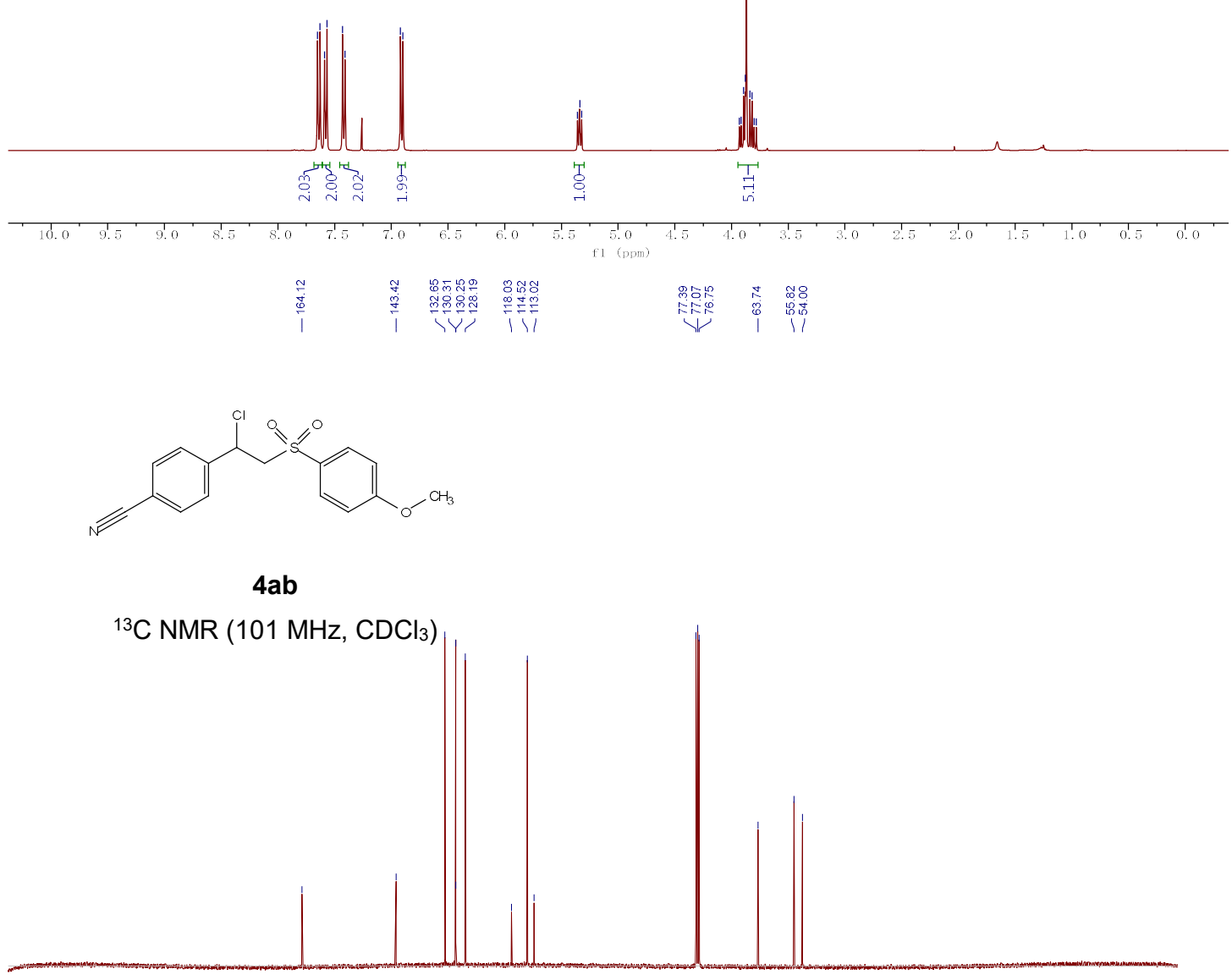

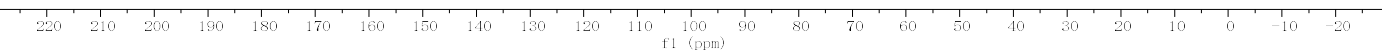

S48 
methyl (S)-4-(1-chloro-2-((4 phenoxyphenyl)sulfonyl)ethyl)benzoate

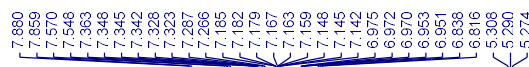

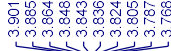

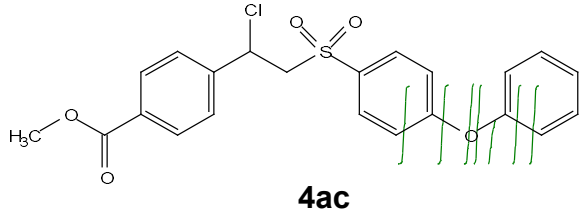

${ }^{1} \mathrm{H}$ NMR $\left(400 \mathrm{MHz}, \mathrm{CDCl}_{3}\right)$

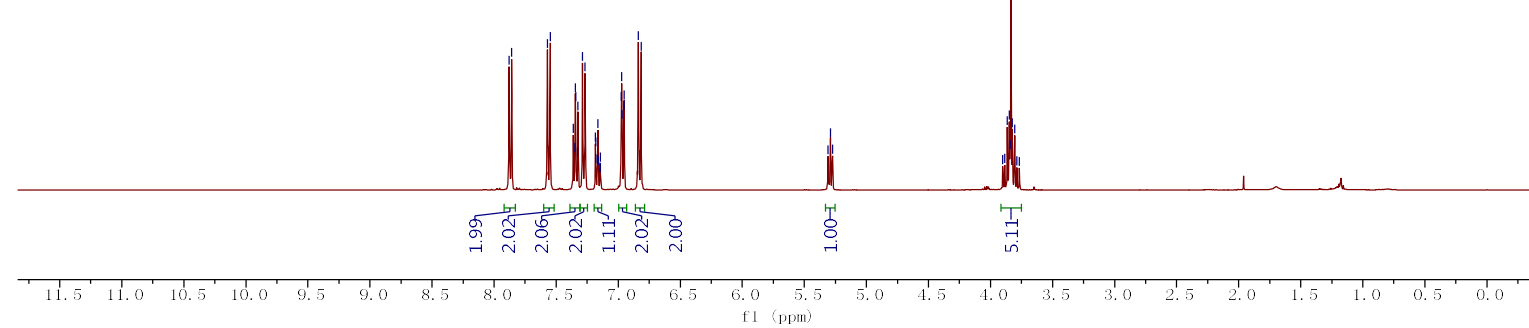

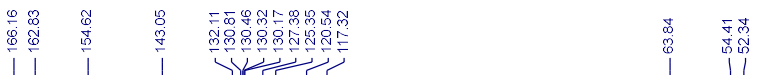

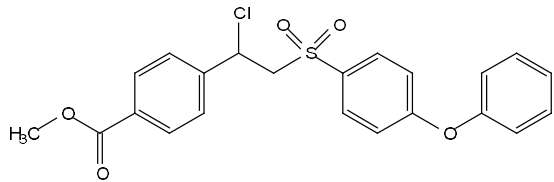

$4 a c$

${ }^{13} \mathrm{C}$ NMR (101 MHz, $\left.\mathrm{CDCl}_{3}\right)$
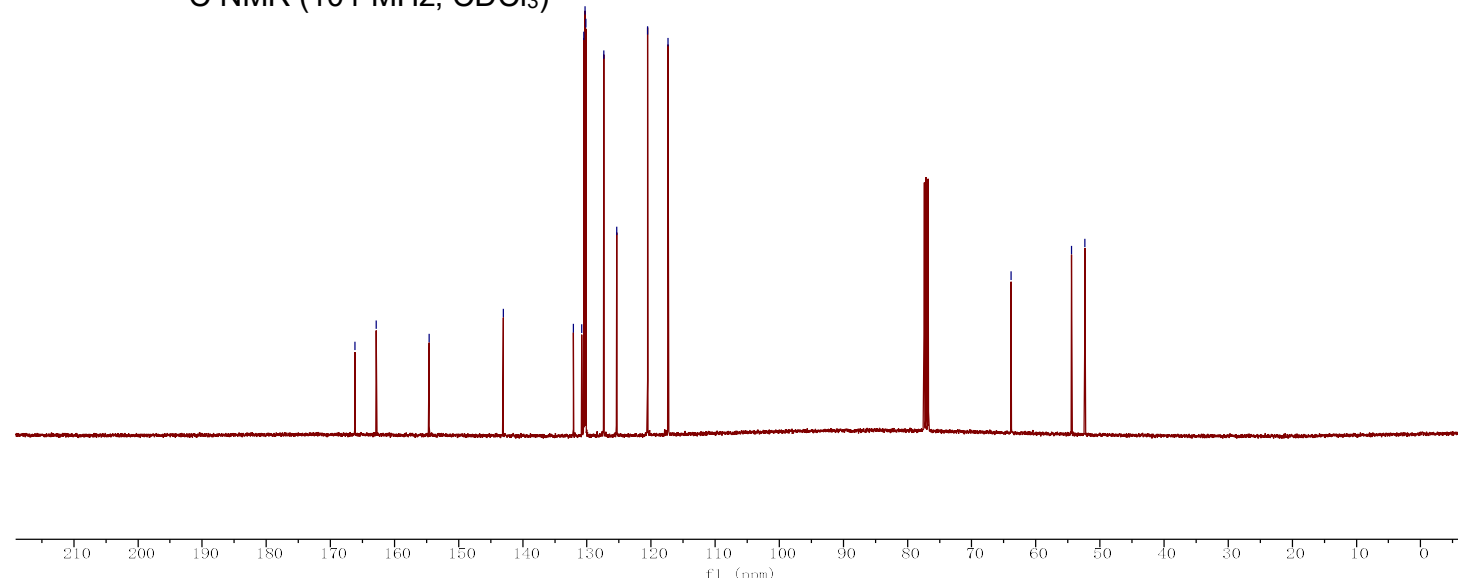
(S)-1-(1-chloro-2-((4-methoxyphenyl)sulfonyl)ethyl)-4-(trifluoromethyl)benzene

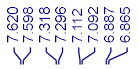

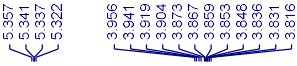

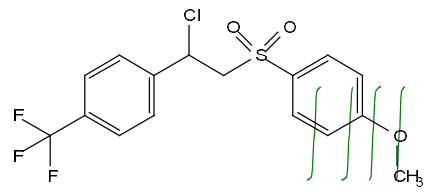

4ad

${ }^{1} \mathrm{H}$ NMR $\left(400 \mathrm{MHz}, \mathrm{CDCl}_{3}\right)$
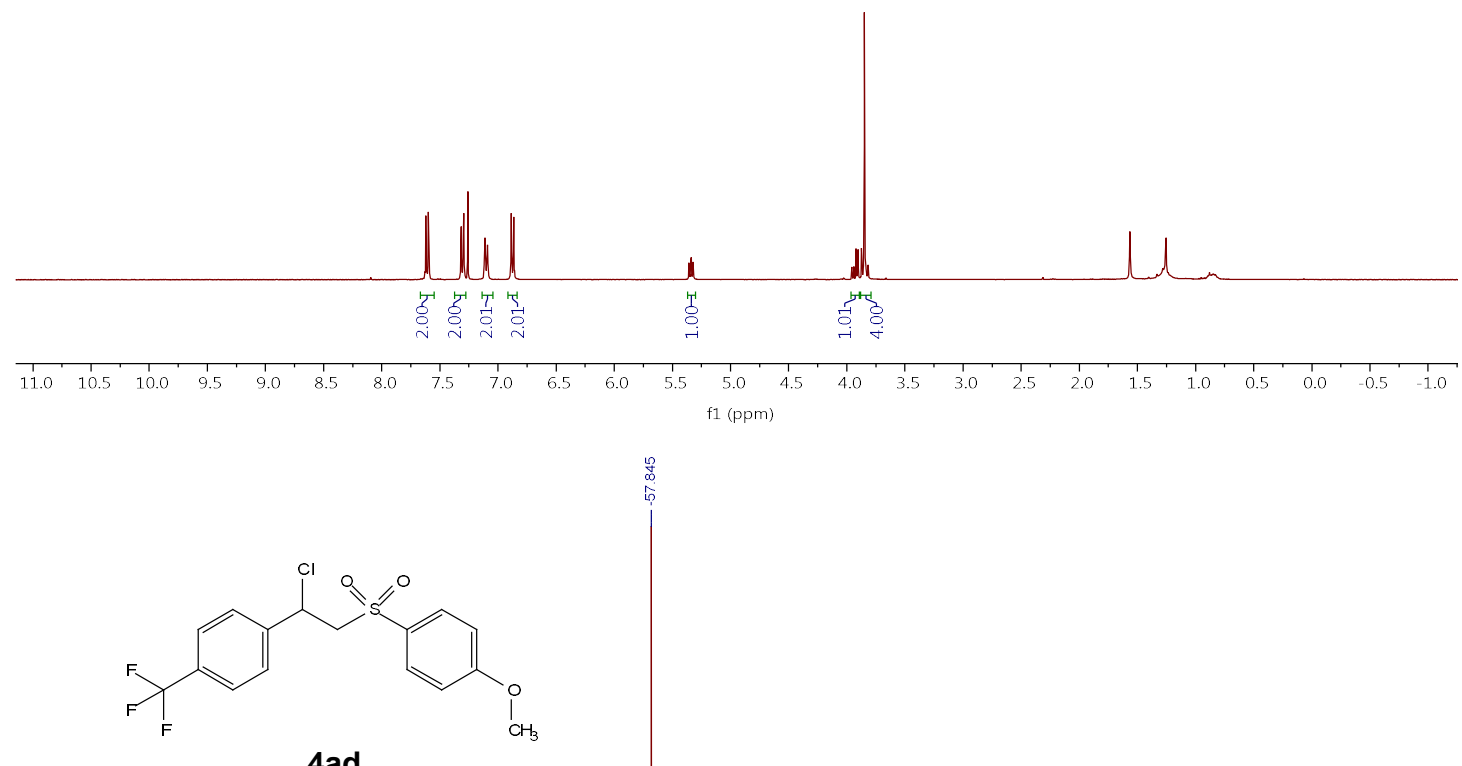

4ad

${ }^{19}$ F NMR (376 MHz, $\left.\mathrm{CDCl}_{3}\right)$

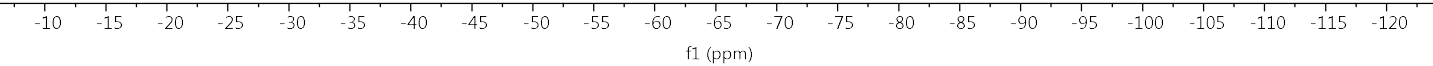




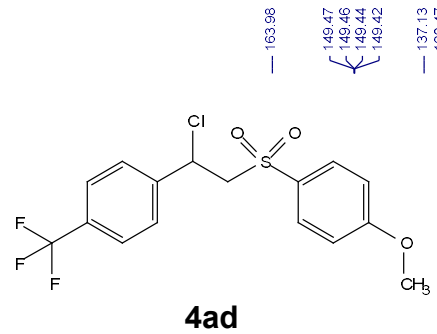

${ }^{13} \mathrm{C}$ NMR (101 MHz, CDCl 3 )

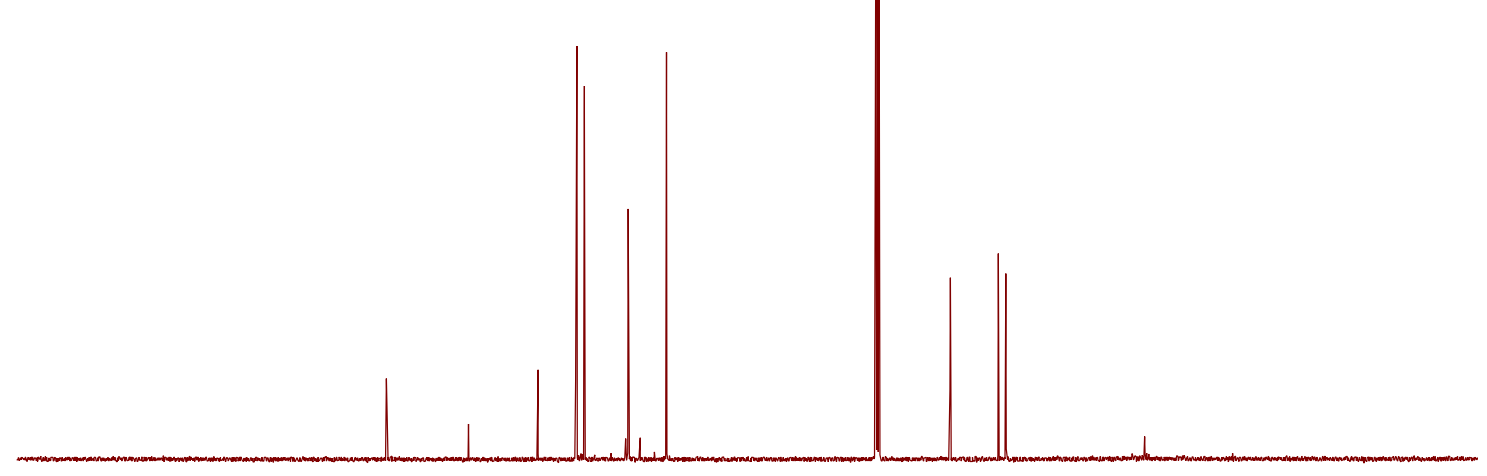

$\begin{array}{llllllllllllllllllllllllll}220 & 210 & 200 & 190 & 180 & 170 & 160 & 150 & 140 & 130 & 120 & 110 & \begin{array}{c}100 \\ \mathrm{f} 1(\mathrm{ppm})\end{array} & 90 & 80 & 7 \mathrm{C} & 60 & 5 \mathrm{C} & 40 & 30 & 20 & 10 & 0 & -10 & -20\end{array}$ 
(S)-7-(1-chloro-2-((4-phenoxyphenyl)sulfonyl)ethyl)-2H-chromen-2-one

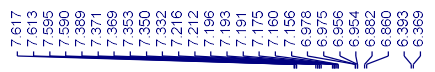

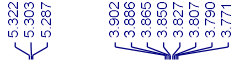

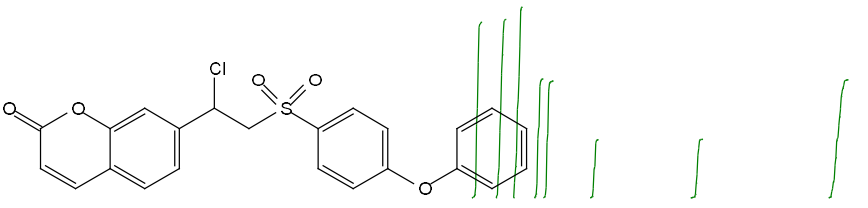

$4 a e$

${ }^{1} \mathrm{H}$ NMR $\left(400 \mathrm{MHz}, \mathrm{CDCl}_{3}\right)$
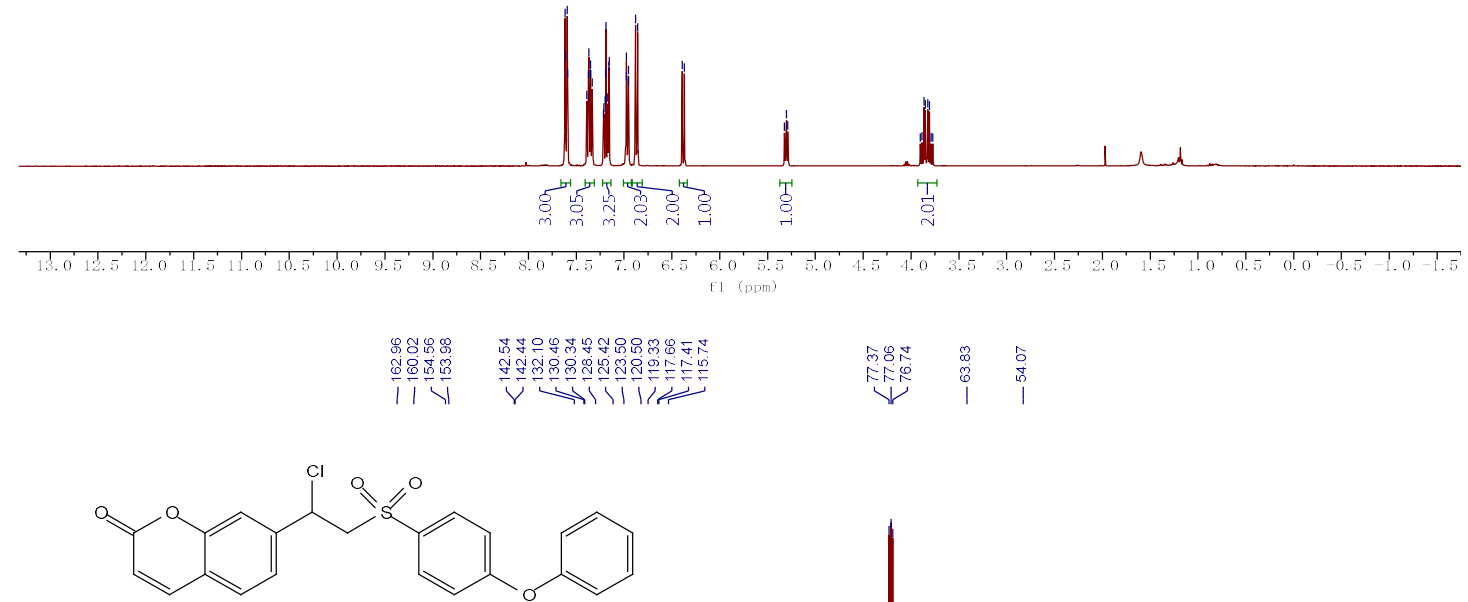

4ae

${ }^{13} \mathrm{C}$ NMR (101 MHz, $\mathrm{CDCl}_{3}$ )

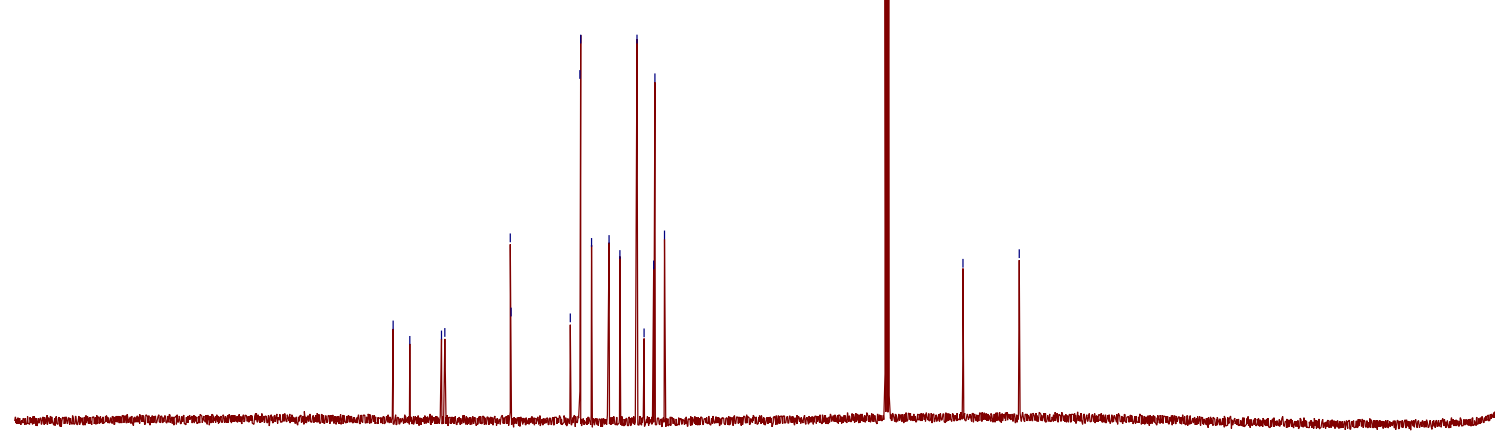


(S)-2-(2-([1,1'-biphenyl]-4-ylsulfonyl)-1-chloroethyl)benzofuran

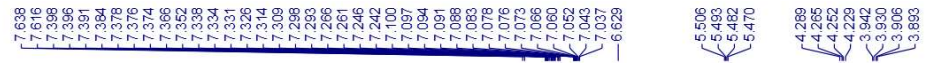

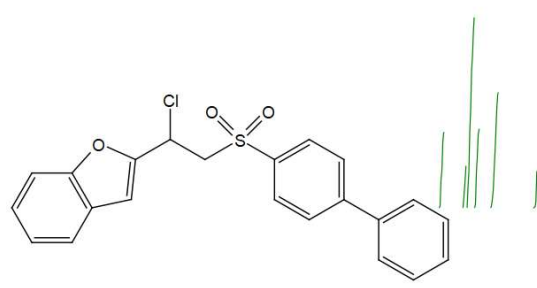

4af

${ }^{1} \mathrm{H}$ NMR $\left(400 \mathrm{MHz}, \mathrm{CDCl}_{3}\right)$

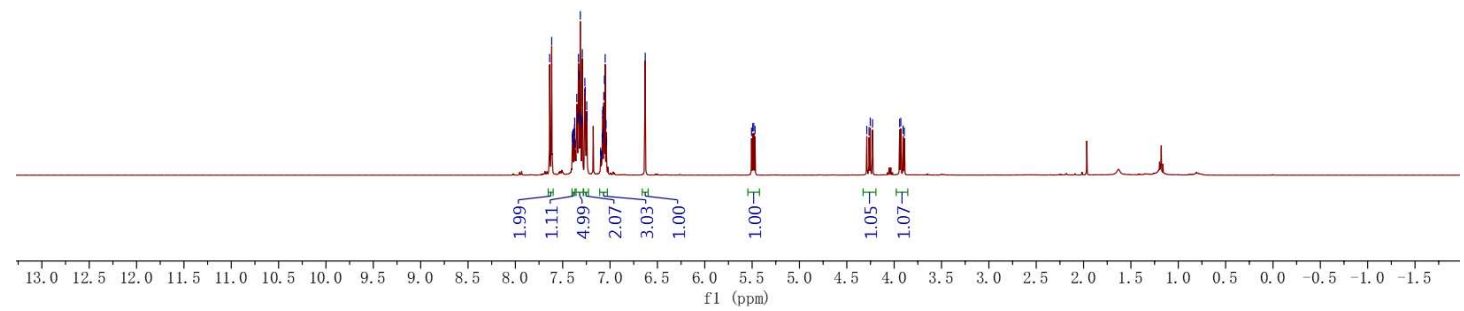

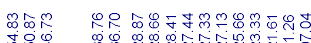

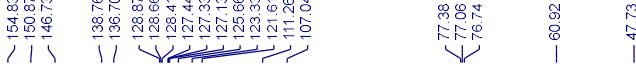

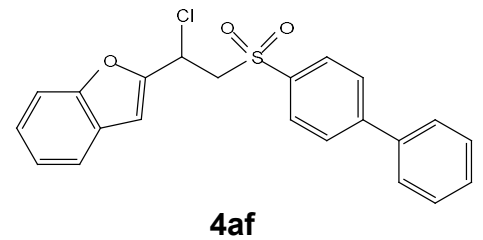

${ }^{13} \mathrm{C}$ NMR $\left(101 \mathrm{MHz}, \mathrm{CDCl}_{3}\right)$

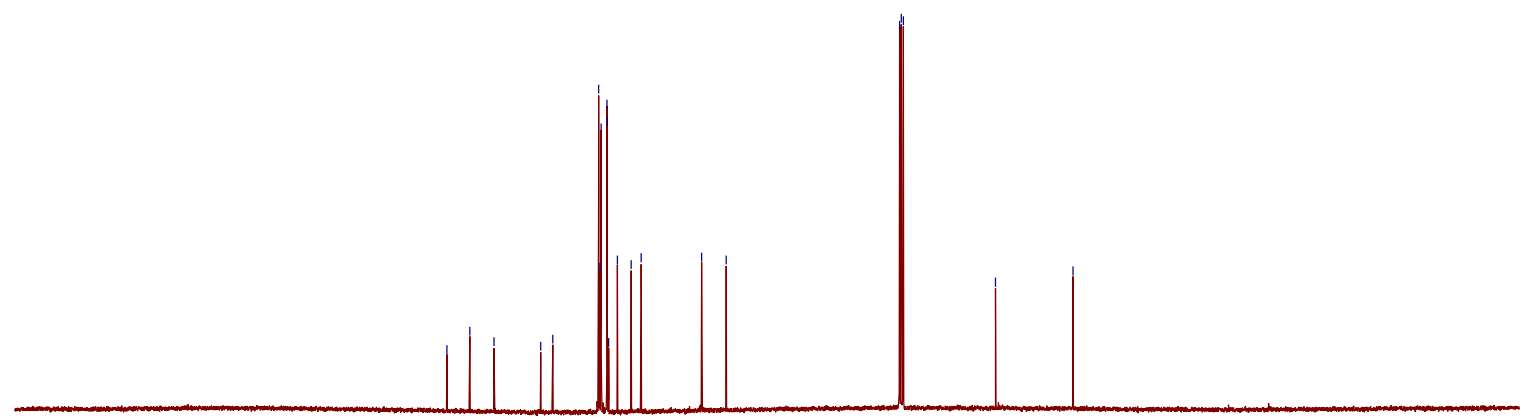


methyl

2-((tert-butoxycarbonyl)amino)-3-(4-((R)-1-chloro-2-((4-

methoxyphenyl)sulfonyl)ethyl)phenyl)propanoate

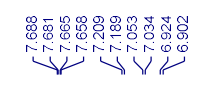
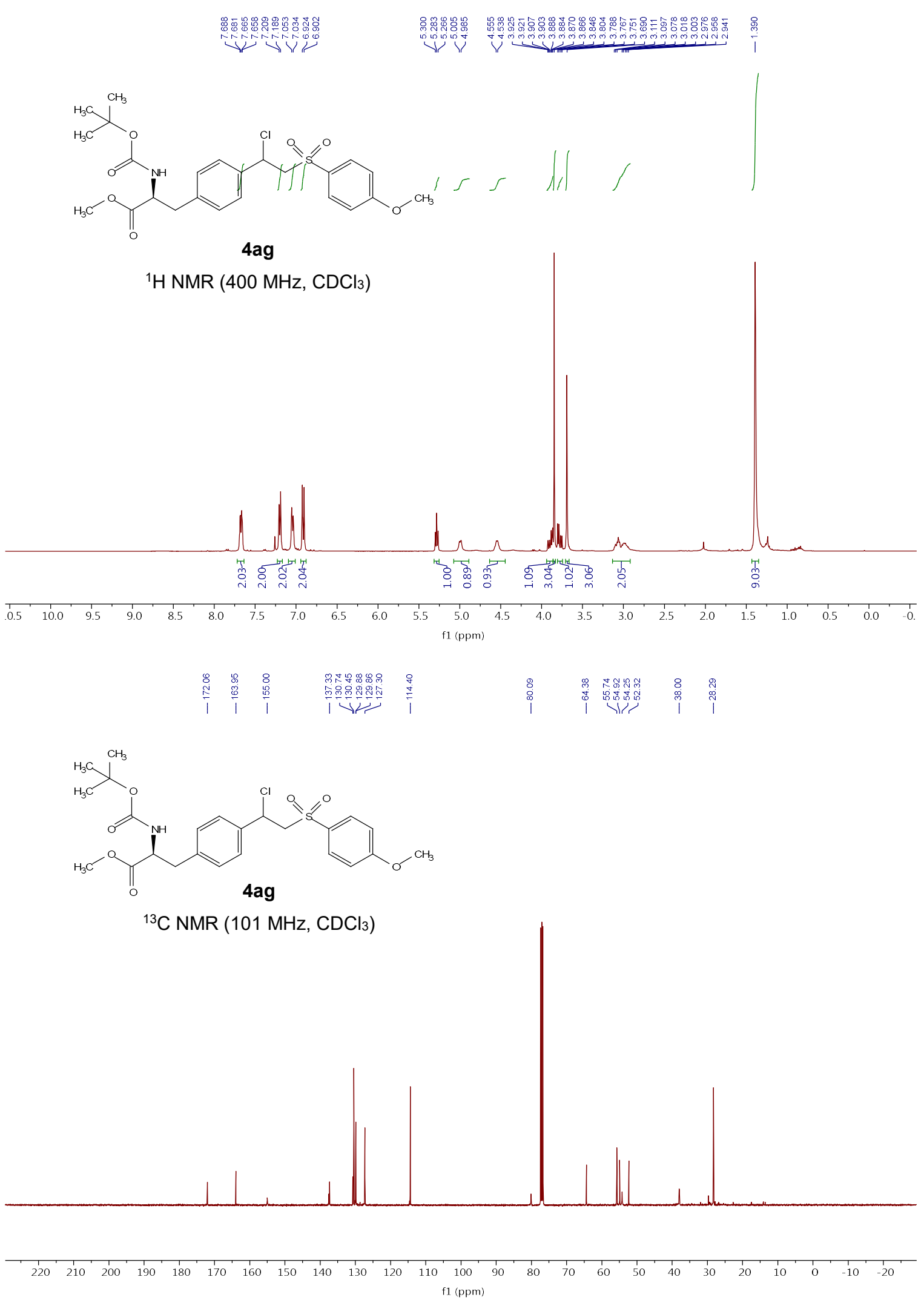

S54 
1-methyl-4-(phenethylsulfonyl)benzene

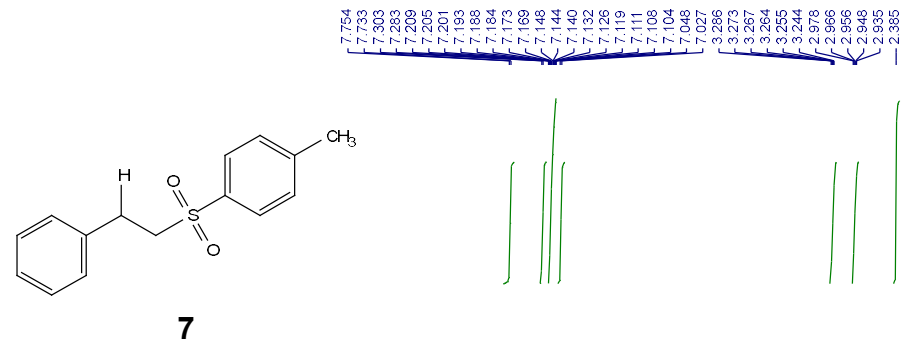

${ }^{1} \mathrm{H}$ NMR (400 MHz, $\left.\mathrm{CDCl}_{3}\right)$
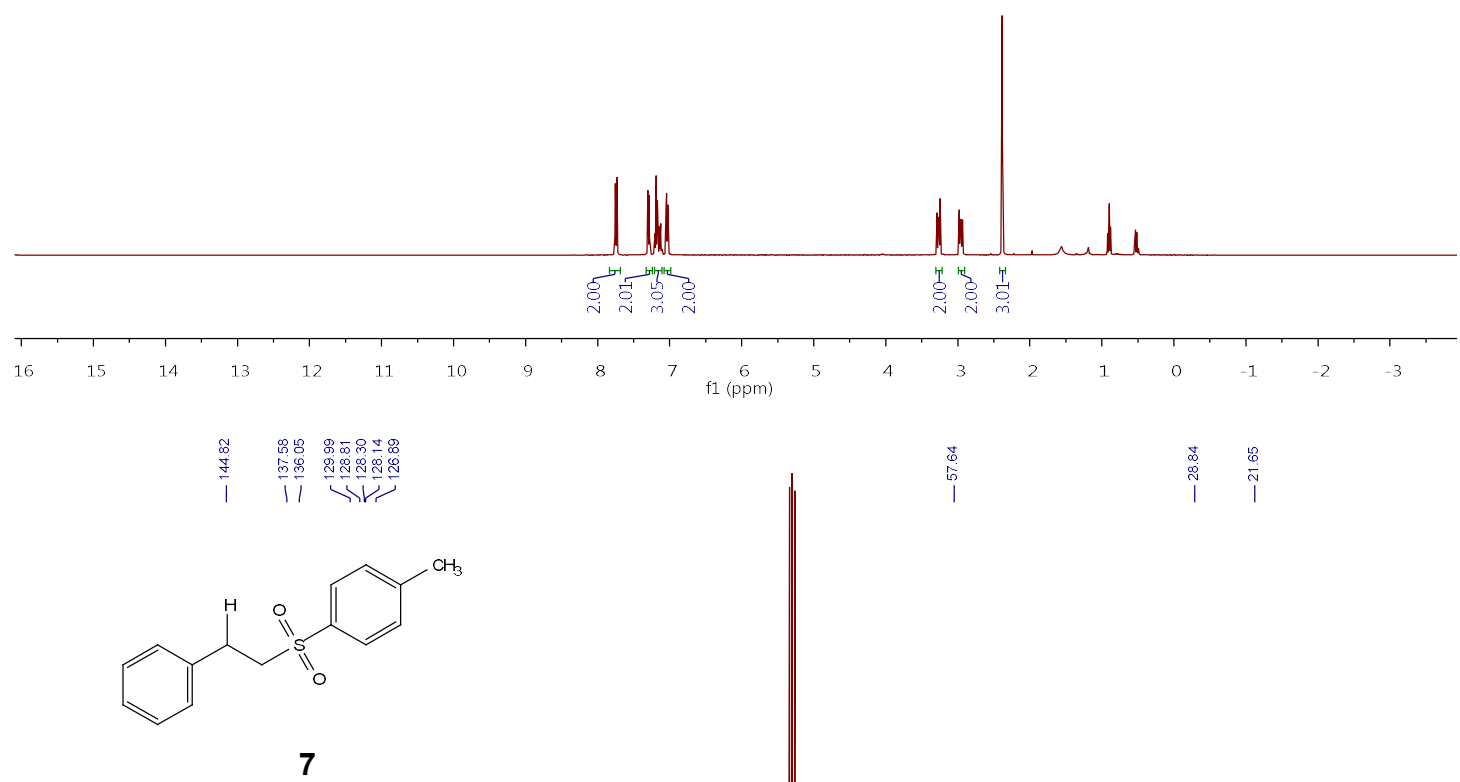

${ }^{13} \mathrm{C}$ NMR $\left(101 \mathrm{MHz}, \mathrm{CDCl}_{3}\right)$
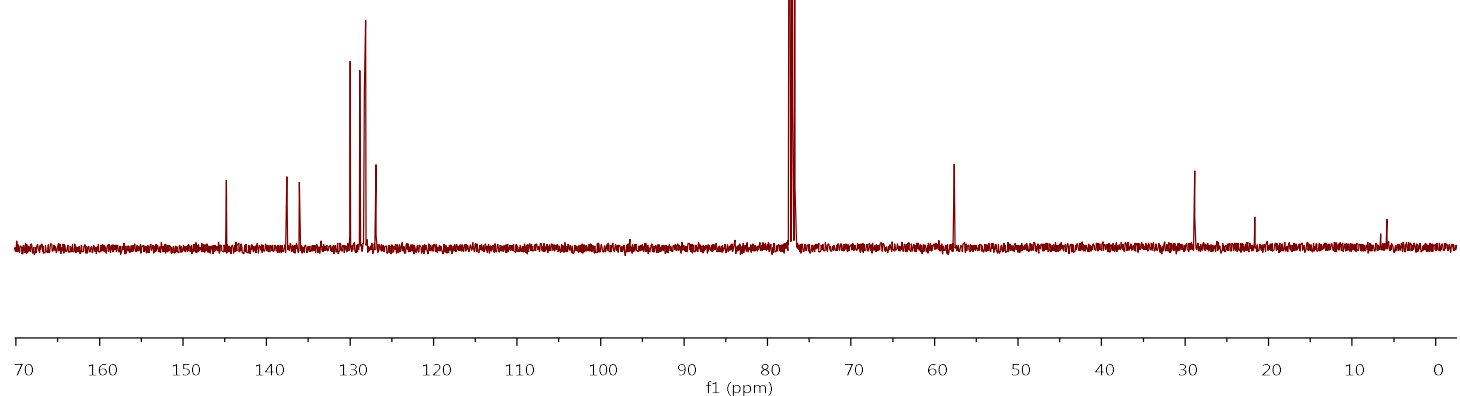
phenyl-2-tosylethan-1-ol

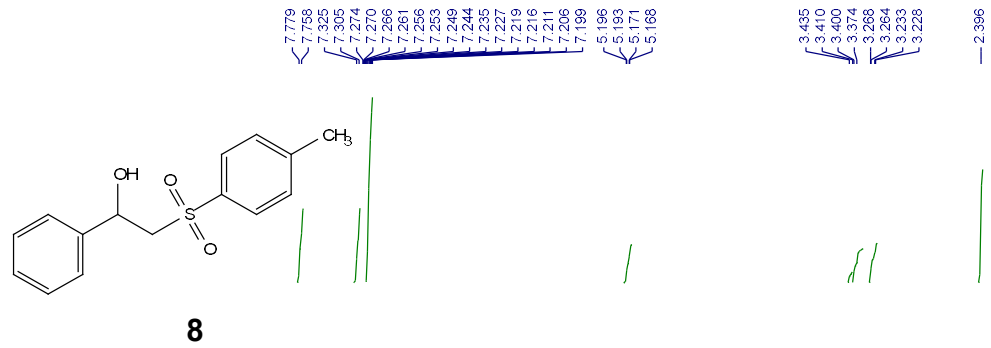

${ }^{1} \mathrm{H}$ NMR $\left(400 \mathrm{MHz}, \mathrm{CDCl}_{3}\right)$
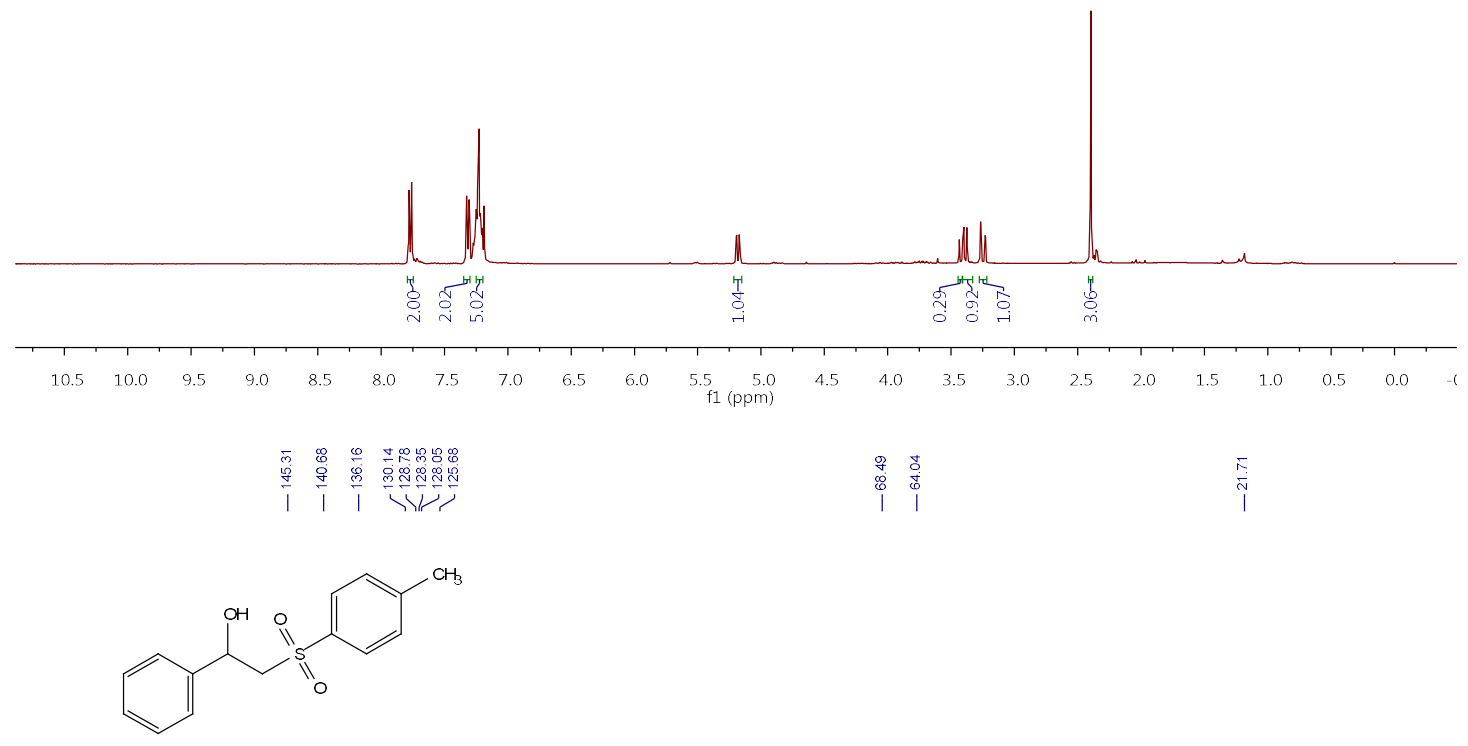

8

${ }^{13} \mathrm{C}$ NMR $\left(101 \mathrm{MHz}, \mathrm{CDCl}_{3}\right)$
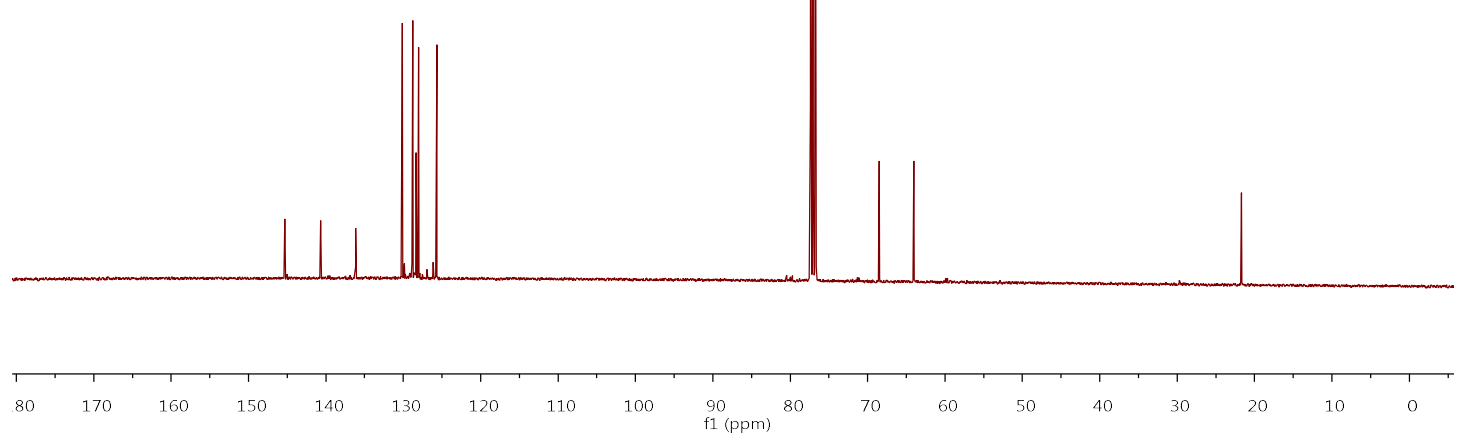